\title{
Modelos de regressão sobre dados composicionais
}

\author{
André Pierro de Camargo
}

\author{
DISSERTAÇÃO APRESENTADA \\ $\mathrm{AO}$ \\ Instituto de Matemática e Estatística \\ DA \\ Universidade De SÃo Paulo \\ PARA \\ OBTENÇÃO DO TÍTULO \\ $\mathrm{DE}$ \\ Mestre em CiÊnCIAS \\ Programa: Matemática Aplicada \\ Orientador: Prof. Dr. Marcelo de Souza Lauretto
}

Durante o desenvolvimento deste trabalho o autor recebeu auxílio financeiro da CAPES 


\section{Modelos de regressão sobre dados composicionais}

Esta versão definitiva da dissertação contém as correções e alterações sugeridas pela Comissão Julgadora durante a defesa realizada por André Pierro de Camargo em 9/12/2011.

Comissão Julgadora:

- Prof. Dr. Marcelo de Souza Lauretto (orientador) - EACH-USP

- Profa. Dra. Delhi Paiva Salinas - EACH-USP

- Prof. Dr. Júlio Michael Stern - IME-USP 


\section{Agradecimentos}

- Agradeço primeiramente aos meus pais por terem me proporcionado um ambiente familiar favorável ao meu desenvolvimento intelectual.

- Agradeço aos meus amigos pelos momentos de descontração necessários para o relaxamento da mente.

- Agradeço aos meus professores pelo conhecimento adquirido direta ou indiretamente, em especial meu orientador (Marcelo Lauretto), o professor Júlio Michael Stern e meus ex-orientadores de iniciação científica: Eduardo do Nascimento Marcos e Paulo Agozzini Martin.

- Agradeço à minha esposa, Roberta, e à minha pequena filha, Marina, pelo apoio e carinho recebidos nesse período.

- Agraddeço a CAPES pelo auxílio financeiro. 


\section{Resumo}

Dados composicionais são constituídos por vetores cujas componentes representam as proporções de algum montante, isto é: vetores com entradas positivas cuja soma é igual a 1. Em diversas áreas do conhecimento, o problema de estimar as partes $y_{1}, y_{2}, \ldots, y_{D}$ correspondentes aos setores $S E_{1}, S E_{2}, \ldots, S E_{D}$, de uma certa quantidade $Q$, aparece com frequência. As porcentagens $y_{1}, y_{2}, \ldots, y_{D}$ de intenção de votos correspondentes aos candidatos $C a_{1}, C a_{2}, \ldots, C a_{D}$ em eleições governamentais ou as parcelas de mercado correspondentes a industrias concorrentes formam exemplos típicos. Naturalmente, é de grande interesse analisar como variam tais proporções em função de certas mudanças contextuais, por exemplo, a localização geográfica ou o tempo. Em qualquer ambiente competitivo, informações sobre esse comportamento são de grande auxílio para a elaboração das estratégias dos concorrentes.

Neste trabalho, apresentamos e discutimos algumas abordagens propostas na literatura para regressão sobre dados composicionais, assim como alguns métodos de seleção de modelos baseados em inferência bayesiana.

Palavras-chave: Modelos de regressão, Dados composicionais, Seleção de modelos, BIC, FBST. 


\section{Abstract}

Compositional data consist of vectors whose components are the proportions of some whole. The problem of estimating the portions $y_{1}, y_{2}, \ldots, y_{D}$ corresponding to the pieces $S E_{1}, S E_{2}, \ldots, S E_{D}$ of some whole $Q$ is often required in several domains of knowledge. The percentages $y_{1}, y_{2}, \ldots, y_{D}$ of votes corresponding to the competitors $C a_{1}, C a_{2}, \ldots, C a_{D}$ in governmental elections or market share problems are typical examples. Of course, it is of great interest to study the behavior of such proportions according to some contextual transitions. In any competitive environmet, additional information of such behavior can be very helpful for the strategists to make proper decisions.

In this work we present and discuss some approaches proposed by different authors for compositional data regression as well as some model selection methods based on bayesian inference.

Keywords: Regression models, Compositional data, Model selection, BIC, FBST. 


\section{Sumário}

$\begin{array}{ll}\text { Lista de Abreviaturas } & \text { ix }\end{array}$

Lista de Símbolos $\quad$ xi

Lista de Figuras $\quad$ xiii

Lista de Tabelas $\quad$ xv

1 Introdução $\quad 1$

1.1 Preliminares . . . . . . . . . . . . . . . . . . . . 3

2 Modelos de regressão de Dirichlet $\quad 5$

2.1 A distribuição de Dirichlet . . . . . . . . . . . . . . . . . . . 5

2.2 Regressão de Dirichlet linear . . . . . . . . . . . . . . . . . . . . . . 6

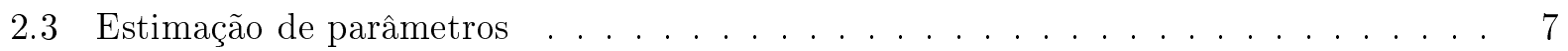

2.3.1 Algoritmo de Hijazi-Jernigan para seleção de valores iniciais . . . . . . . . 8

2.3.2 Novo algoritmo para seleção de valores iniciais . . . . . . . . . . . . . 8

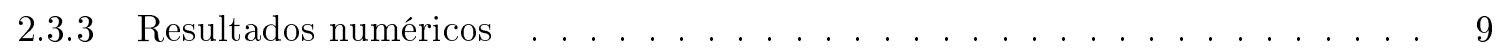

2.4 Eliminação das restrições sobre o espaço paramétrico . . . . . . . . . . . . . . . . 10

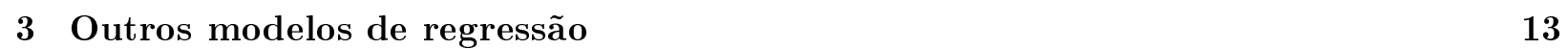

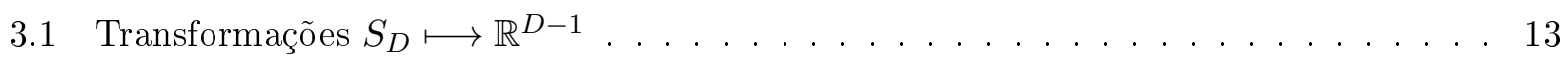

3.1.1 Abrangência do método . . . . . . . . . . . . . . . . . . . . . . 14

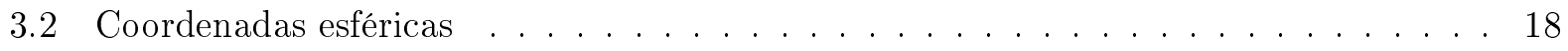

3.3 Outras transformações no Simplex . . . . . . . . . . . . . . . . . . 20

3.3 .1 Observações . . . . . . . . . . . . . . . . . . 23

3.4 Método dos mínimos quadrados no Simplex . . . . . . . . . . . . . . . . 23

4 Aplicações $\quad 25$

4.1 Lago Ártico . . . . . . . . . . . . . . . . . . . . . . . . . 25

4.1 .1 Modelo de Dirichlet Linear . . . . . . . . . . . . . . . . 25

4.1 .2 Modelo de Melo, Vasconcellos e Lemonte . . . . . . . . . . . . . . . . . 26

4.1.3 Modelo linear Logaritmo da Razão/ Log-esférico . . . . . . . . . . . . . . . . 27

4.1 .4 Modelo linear Tangente-esférico . . . . . . . . . . . . . . . . . 28

4.2 Despesas domésticas (bivariado) . . . . . . . . . . . . . . . . . 29

4.2 .1 Modelo de Dirichlet Linear . . . . . . . . . . . . . . . . . . . . 29 
4.2.2 Modelo de Melo, Vasconcellos e Lemonte . . . . . . . . . . . . . . . . . . . . 30

4.2 .3 Modelo linear TgRatio (3.22) . . . . . . . . . . . . . . . . . . 31

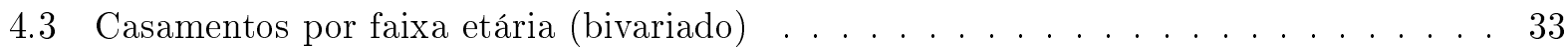

4.3.1 Modelo de Dirichlet Linear . . . . . . . . . . . . . . . . . . . . . . 33

4.3.2 Modelo de Melo, Vasconcellos e Lemonte . . . . . . . . . . . . . . . . . . . . 34

4.3.3 Modelo linar Log-esférico _ . . . . . . . . . . . . . . . . . . . 35

4.4 Considerações sobre o Espaço Paramétrico . . . . . . . . . . . . . . . . . . 36

4.4.1 Modelo de Dirichlet/ Mínimos quadrados (linear) . . . . . . . . . . . . . 36

5 Seleção de modelos $\quad 39$

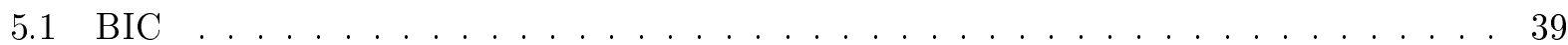

5.1 .1 Uma proposta de formalização do $B I C \ldots \ldots \ldots \ldots$. . . . . . . . 42

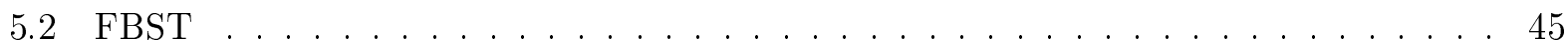

5.2 .1 Definição formal . . . . . . . . . . . . . . . . . . . 45

5.3 Teste da Razão de verossimilhança $(\mathrm{LR}) \ldots \ldots \ldots$. . . . . . . . . . 46

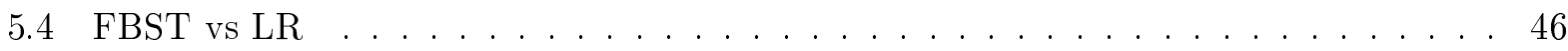

5.5 Aplicações do BIC aos modelos de Dirichlet linear . . . . . . . . . . . . . . . . . . . 47

5.5 .1 Lago Ártico . . . . . . . . . . . . . . . . . . . . . 47

5.5 .2 Despesas domésticas . . . . . . . . . . . . . . . . . . 48

6 Conclusões $\quad 51$

A Cálculo dos coeficientes da regressão linear por mínimos quadrados 53

B Base de dados $\quad 55$

B.1 Lago Ártico . . . . . . . . . . . . . . . . . . . . . . . . . . . . 56

B.2 Despesas Domésticas . . . . . . . . . . . . . . . . . . 57

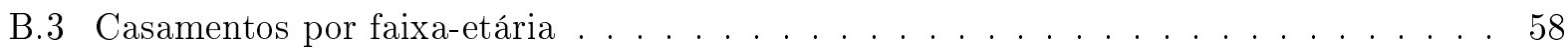

Referências Bibliográficas $\quad 59$

$\begin{array}{ll}\text { Índice Remissivo } & 61\end{array}$ 


\section{Lista de Abreviaturas}

BIC Critério de Informação Bayesiano (Bayesian information criterion).

FBST Teste de significância integralmente bayesiano (Full Bayesian Significant Test).

LR Teste da Razão de verossimilhança (Likelihood-ratio test). 


\section{Lista de Símbolos}

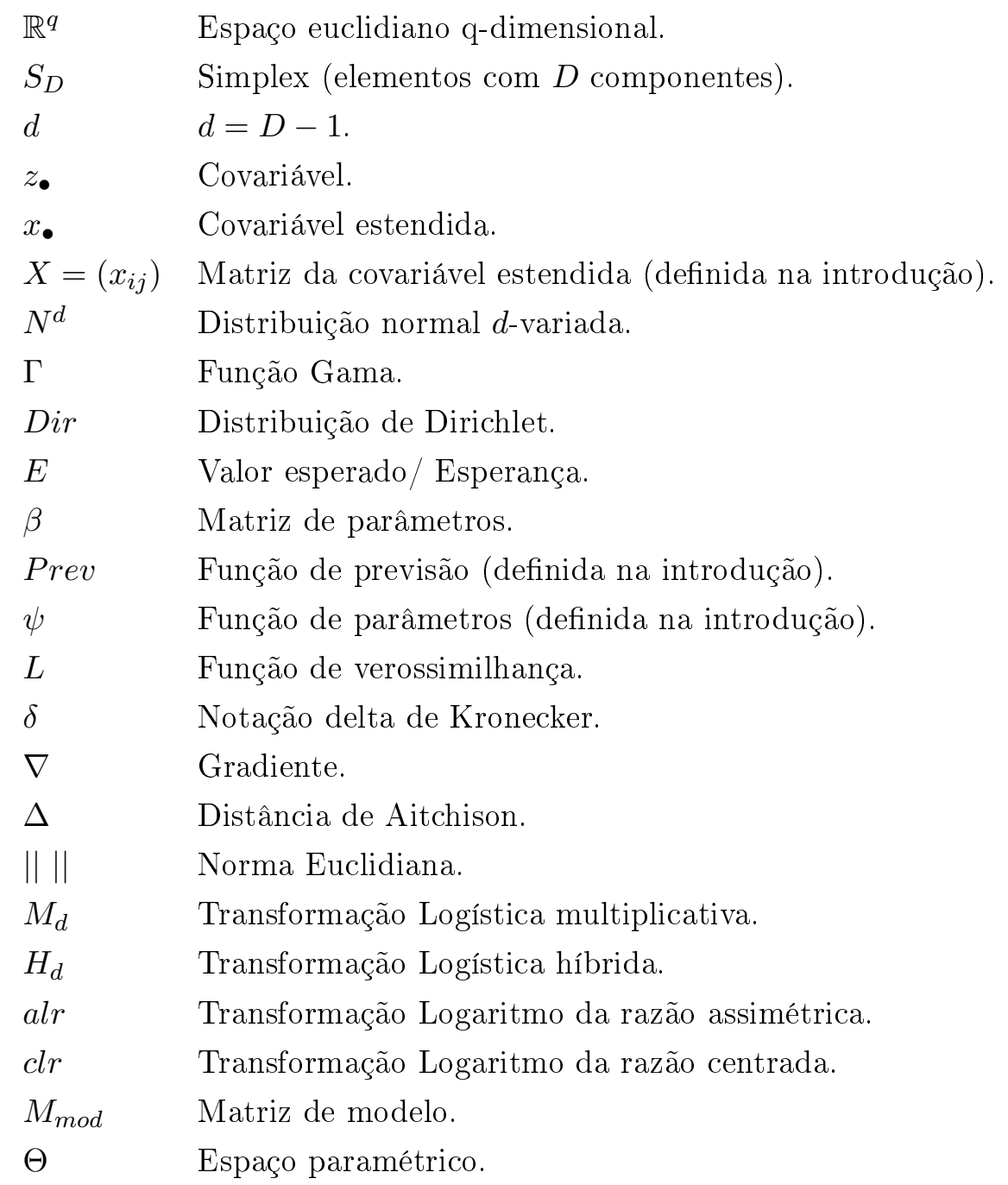




\section{Lista de Figuras}

2.1 Comparativo entre os métodos de seleção de valores iniciais para o modelo de Dirichlet linear................................ 10

3.1 Interpretação geométrica da transformação alr . . . . . . . . . . . . . . . . . . . . . 20

3.2 Parametrização do 3-Simplex por coordenadas esféricas . . . . . . . . . . . . . . . . . 21

4.1 Modelos de Dirichlet lineares . . . . . . . . . . . . . . . . . . . . . 26

4.2 Modelos de Melo, Vasconcellos e Lemonte. . . . . . . . . . . . . . . . . . . . . . . . . 26

4.3 Modelo linear Log-esférico vs Modelo linear Logaritmo da razão . . . . . . . . . . . . 28

4.4 Modelos Tangente-esféricos . . . . . . . . . . . . . . . . . . . . . . . . . . . . 29

4.5 Modelo de Dirichlet linear . . . . . . . . . . . . . . . . . . . . . . . . 30

4.6 Modelo de Melo, Vasconcellos e Lemonte . . . . . . . . . . . . . . . . . . . . . . . . . 31

4.7 Modelo linear TgRatio . . . . . . . . . . . . . . . . . . . . . . . 32

4.8 Modelo de Dirichlet Linear . . . . . . . . . . . . . . . . . . . . . . . . . . . 33

4.9 Modelo de Melo, Vasconcellos e Lemonte . . . . . . . . . . . . . . . . . . . . . . . . . 34

4.10 Modelo Log-esférico . . . . . . . . . . . . . . . . . . . . . . . . . . . . . . 36

4.11 Sensibilidade da verossimilhança de MDQ sobre dilatações . . . . . . . . . . . . . . 37

4.12 Modelos quadráticos restritos à casca esférica unitária . . . . . . . . . . . . . . . . 38

5.1 (a) Erro Tipo I, (b) Erro Tipo II, (c) Erro médio e (d) Erro Tipo II empírico. . . . . 47

5.2 Submodelos de MDQ . . . . . . . . . . . . . . . . . . . . . . . . 48

5.3 Melhor modelo segundo Schwarz . . . . . . . . . . . . . . . . . . . . . . . 49 


\section{Lista de Tabelas}

3.1 Transformações logísticas elementares $y \in S_{D} \longmapsto v \in \mathbb{R}^{d} \ldots \ldots \ldots \ldots$

4.1 Parâmetros de máxima verossimilhança . . . . . . . . . . . . . . . 26

4.2 Parâmetros de máxima verossimilhança . . . . . . . . . . . . . . 27

4.3 Parâmetros de máxima verossimilhança . . . . . . . . . . . . . . . 28

4.4 Parâmetros de máxima verossimilhança . . . . . . . . . . . . . . . . . . 29

4.5 Parâmetros de máxima verossimilhança . . . . . . . . . . . . . . . 30

4.6 Parâmetros de máxima verossimilhança . . . . . . . . . . . . . . . 31

4.7 Parâmetros de máxima verossimilhança . . . . . . . . . . . . . . . . 32

4.8 Parâmetros de máxima verossimilhança . . . . . . . . . . . . . . . . 34

4.9 Parâmetros de máxima verossimilhança $\ldots \ldots \ldots \ldots \ldots \ldots$

4.10 Parâmetros de máxima verossimilhança . . . . . . . . . . . . . . . . 35

4.11 Transformação de MDQ para coordenadas esféricas . . . . . . . . . . . . . . 38

4.12 Parâmetros de máxima verossimilhança/ Mínimos quadrados . . . . . . . . . . . 38

5.1 Alguns submodelos de MDQ ordenados do menor para o maior BIC . . . . . . . . . 48

B.1 Composição do solo do Lago Ártico em função da profundidade. . . . . . . . . . . . 56

B.2 Despesas domésticas: $\mathrm{T}=$ Total gasto $(\mathrm{em} \mathrm{HK} \$)$; $\mathrm{A}=$ Alimentação; $\mathrm{O}=$ Outros Bens; $S=$ Serviços. . . . . . . . . . . . . . . . 57

B.3 Porcentagen de casamentos por faixa etária e sexo. Fonte: IBGE . . . . . . . . . . . 58 


\section{Capítulo 1}

\section{Introdução}

Em diversas áreas do conhecimento, o problema de estimar as partes $y_{1}, y_{2}, \ldots, y_{D}$ correspondentes aos setores $S E_{1}, S E_{2}, \ldots, S E_{D}$, de uma certa quantidade $Q$, aparece com frequência. As porcentagens $y_{1}, y_{2}, \ldots, y_{D}$ de intenção de votos correspondentes aos candidatos $C a_{1}, C a_{2}, \ldots, C a_{D}$ em eleições governamentais ou as parcelas de mercado correspondentes a industrias concorrentes formam exemplos típicos. Naturalmente, é de grande interesse analisar como variam tais proporções em função de certas mudanças contextuais, por exemplo, a localização geográfica ou o tempo. Em qualquer ambiente competitivo, informações sobre esse comportamento são de grande auxílio para a elaboração das estratégias dos competidores.

Definição: Chamamos de um dado composicional a cada $D$-upla $\left(y_{1}, y_{2}, \ldots, y_{D}\right)$ de números positivos tais que $\sum_{j=1}^{D} y_{j}=1$. O conjunto

$$
S_{D}=\left\{\left(y_{1}, y_{2}, \ldots, y_{D}\right) \in \mathbb{R}^{D}: \sum_{j=1}^{D} y_{j}=1, y_{j}>0, j=1,2, \ldots, D\right\}
$$

é denominado o $D-1$-Simplex.

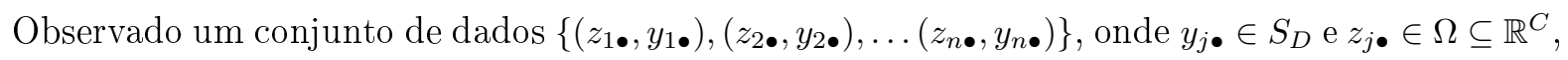
$j=1,2, \ldots, n$, deseja-se obter um modelo de regressão paramétrico $y_{\bullet} \sim z_{\bullet}$. O sucesso dessa tarefa depende, essencialmente, de dois fatores: da escolha da família paramétrica de modelos e do método de inferência utilizado. Um método bastante utilizado consiste em:

1. Selecionar uma família paramétrica de modelos probabilísticos $G=\left\{g\left(y_{\bullet} \mid \psi\right): \psi \in \Psi\right\}$ (aqui descritos por suas funções densidade de probabilidade), definidos em $S_{D}$.

2. Supor que, para cada valor de $z_{\bullet}, y_{\bullet} \mid z_{\bullet}$ é distribuída segundo $G$ com parâmetro $\psi\left(z_{\bullet}, \beta\right)$, cuja dependencia com $z_{\bullet}$ é calibrada por um vetor (ou matriz) $\beta$.

3. Ajustar o vetor/matriz de parâmetros $\beta$.

4. Estimar o valor de $y_{\bullet}$ em $z_{\bullet}, \operatorname{Prev}\left(z_{\bullet}\right)$, como o valor esperado da distribuição $G$ com parâmetro $\psi\left(z_{\bullet}, \beta\right)$. 
Assumindo que as variáveis $y_{j} \mid z_{j \bullet}, j=1,2, \ldots, n$ são independentes, podemos formar a função de verossimilhança com respeito ao vetor/matriz de parâmetros $\beta$ para estimar seus valores via máxima verossimilhança, mínimos quadrados ou outro método de inferência.

Neste trabalho investigamos e estendemos algumas abordagens propostas na literatura baseadas no método descrito acima. O texto está assim organizado:

No segundo Capítulo, apresentamos dois modelos de regressão descritos em Campbell e Mosimann (1987) e Melo et al. (2009), ambos baseados na distribuição de Dirichlet (a distribuição mais conhecida definida no Simplex, Pereira e Stern (2008)). A nossa principal contribuição referente ao conteúdo desse Capítulo é o desenvolvimento de um algoritmo mais eficiente para encontrar um ponto inicial viável para ser fornecido aos algoritmos de maximização da função de verossimilhança (Seção 2.3.2).

No terceiro Capítulo, apresentamos os modelos de regressão descritos nos trabalhos de Aitchinson (1986) e Wang et al. (2007). O primeiro propôe uma alternativa à utilização de distribuições no Simplex, transformando os dados composicionais para o Espaço Euclidiano, modelando os dados transformados e retornando para o Simplex. O segundo descreve os dados composicionais em termos de coordenadas esféricas generalizadas e modela os ângulos diretamente em função da covariável $X$. A nossa principal contribuição referente ao conteúdo desse Capítulo consiste na completa caracterização das transformações equivalentes (Seção 3.1.1) e na elaboração de novas transformações entre o Simplex e o Espaço Euclidiano (Seção 3.3).

No quarto Capítulo, aplicamos os modelos de regressão descritos nos Capítulos 2 e 3 a três conjuntos de dados (disponíveis para consulta no Apêndice B):

1. O primeiro é referente a 39 composições do solo do Lago Ártico (areia, lodo e argila em função da profundidade) apresentados por Coakley e Rust Coakley e Rust (1968) e adaptados por Aitchinson (1986). A análise do solo, em geral, é um fator discriminante na área da construção civil.

2. O segundo é referente ao conjunto de dados encontrado em Aitchinson (1986), constituído pelas despesas domésticas (separadas em Manutenção domiciliar, Alimentação, Outros bens e Serviços.) de 20 homens e 20 mulheres, em função do total gasto.

3. O terceiro corresponde a um conjunto de dados fornecido pelo IBGE (Instituto Brasileiro de Geografia e Estatística) e disponível em http://seriesestatisticas.ibge.gov.br/ relativo à variação das porcentagens de casamentos por faixa etária e sexo no período 1984-2002.

A análise desses outros dois conjuntos de dados é de grande interesse para as ciências sociais. O segundo constitui uma típica pesquisa mercadológica e o estudo do terceiro pode auxiliar na descrição de outros fenômenos direta ou indiretamente correlacionados, como, por exemplo, a disseminação de doenças sexualmente transmissíveis.

No quinto Capítulo expomos três métodos de seleção de modelos baseados em inferência bayesiana: BIC, FBST e Teste da razão de verossimilhança. Nossa principal contribuição referente ao conteúdo desse Capítulo é um estudo detalhado do critério de Schwarz (BIC). 


\subsection{Preliminares}

Ao longo do texto, as composições observadas serão denotadas por $y_{j} \bullet=\left(y_{j 1}, y_{j 2}, \ldots, y_{j n}\right), j=$ $1,2, \ldots, n$ e $Y=\left(y_{i j}\right)$ denota a matriz das composições. Um dado composicional genérico (ou variável aleatória assumindo valores no $D$-1-Simplex) será denotado por y ou $y$ o.

Seguindo a notação padrão em modelos de regressão, em lugar da covariável original $z_{\bullet} \in \Omega$, trabalharemos com a variável estendida $x_{\bullet}=\left(f_{1}\left(z_{\bullet}\right), f_{2}\left(z_{\bullet}\right), \ldots, f_{k}\left(z_{\bullet}\right)\right) \in \mathbb{R}^{k}$, onde $f_{1}, f_{2}, \ldots, f_{k}$ : $\Omega \longrightarrow \mathbb{R}$ são transformações apropriadas. Para garantir a unicidade de alguns estimadores ótimos para a matriz $\beta$, iremos assumir que a matriz

$$
X=\left(x_{i j}\right)=\left[\begin{array}{c}
x_{1} \bullet \\
x_{2} \bullet \\
\vdots \\
x_{n} \bullet
\end{array}\right]=\left[\begin{array}{cccc}
f_{1}\left(z_{1} \bullet\right. & f_{2}\left(z_{1} \bullet\right. & \ldots & f_{k}\left(z_{1} \bullet\right) \\
f_{1}\left(z_{2} \bullet\right. & f_{2}\left(z_{2} \bullet\right. & \ldots & f_{k}\left(z_{2} \bullet\right. \\
\vdots & \vdots & & \vdots \\
f_{1}\left(z_{n} \bullet\right. & f_{2}\left(z_{n} \bullet\right. & \ldots & f_{k}\left(z_{n} \bullet\right.
\end{array}\right]
$$

possui posto $k \leq n$ (ver apêndice A).

Para simplificar a notação, vetores do Espaço Euclidiano $\mathbb{R}^{q}$, para $q$ genérico, serão interpretados, eventualmente, como matrizes colunas (quando couber).

Ao longo do texto, $e-s$ e $e+s$ denotarão, respectivamente $10^{-s}$ e $10^{s}$. 


\section{Capítulo 2}

\section{Modelos de regressão de Dirichlet}

\subsection{A distribuição de Dirichlet}

O modelo probabilístico de Dirichlet, $\operatorname{Dir}\left(\lambda_{1}, \lambda_{2}, \ldots, \lambda_{D}\right)$, definido no $D-1$-Simplex, possui função densidade de probabilidade dada por:

$$
f(y \mid \lambda)=\frac{\Gamma(\Lambda)}{\prod_{i=1}^{D} \Gamma\left(\lambda_{i}\right)} \prod_{i=1}^{D} y_{i}^{\lambda_{i}-1}
$$

onde $\Lambda=\sum_{i=1}^{D} \lambda_{i}, \quad \lambda=\left(\lambda_{1}, \lambda_{2}, \ldots, \lambda_{D}\right)>0, \quad y=\left(y_{1}, y_{2}, \ldots, y_{D}\right) \in S_{D}$ e $\Gamma$ representa a função Gama: $\Gamma(t)=\int_{0}^{\infty} u^{t-1} e^{-u} d u$. Fazendo

$$
T(y)=\left(\log \left(y_{1}\right), \log \left(y_{2}\right), \ldots, \log \left(y_{D}\right)\right), \quad A(\lambda-1)=\sum_{i=1}^{D} \log \left(\Gamma\left(\lambda_{i}\right)\right)-\log (\Gamma(\Lambda)),
$$

podemos reescrever (2.1) como $f(y \mid \alpha)=\exp \left\{T(y) \times \alpha^{t}-A(\alpha)\right\}, \alpha=\left(\lambda_{1}-1, \lambda_{2}-1, \ldots, \lambda_{D}-1\right)$,

donde segue que Dir pertence à Familia Exponencial.

Dada uma amostra aleatória $y_{1} \bullet, y_{2} \bullet, \ldots, y_{n} \bullet$ de uma variável $y \sim \operatorname{Dir}\left(\lambda_{1}, \lambda_{2}, \ldots, \lambda_{D}\right)$, a função de máxima verossimilhança é dada por

$$
L\left(\lambda \mid y_{1} \bullet, y_{2} \bullet, \ldots, y_{n} \bullet\right)=\exp \left\{\left[\sum_{j=1}^{n} T\left(Y_{j}\right)\right] \times \alpha^{t}-n . A(\alpha)\right\} .
$$

A demonstração da existência e unicidade do estimador de máxima verrosimilhança para a Distribuição de Dirichlet pode ser encontradas em Ronning (1989). Demais propriedades da distribuição de Dirichlet podem ser encontradas em Pereira e Stern (2008). 


\subsection{Regressão de Dirichlet linear}

No modelo de regressão de Dirichlet linear, supõe-se que $y_{\bullet} \mid x_{\bullet}$ possui distribuição de Dirichlet com parâmetros dados por

$$
\psi\left(x_{\bullet}, \beta\right)=\left[\begin{array}{c}
\lambda_{1}\left(x_{\bullet}\right) \\
\lambda_{2}\left(x_{\bullet}\right) \\
\vdots \\
\lambda_{D}\left(x_{\bullet}\right)
\end{array}\right]=\left[\begin{array}{cccc}
m_{11} \beta_{11} & m_{12} \beta_{12} & \ldots & m_{1 k} \beta_{1 k} \\
m_{21} \beta_{21} & m_{22} \beta_{22} & \ldots & m_{2 k} \beta_{2 k} \\
\vdots & \vdots & & \vdots \\
m_{D 1} \beta_{D 1} & m_{D 2} \beta_{D 2} & \ldots & m_{D k} \beta_{D k}
\end{array}\right] \times x_{\bullet}
$$

onde a matriz de modelo $M_{\text {mod }}=\left(m_{i j}\right)$ indica quais dos parâmetros $\beta_{i j}$ fazem parte do modelo, isto é:

$$
\begin{cases}m_{i j}=1, & \text { se } \beta_{i j} \text { pertence ao modelo. } \\ m_{i j}=0, & \text { caso contrário. }\end{cases}
$$

A matriz de modelo possui um papel importante na identificação das variáveis relevantes do modelo (ver Capítulo 5). Neste Capítulo, porém, vamos assumir (a menos que seja mencionado) que a matriz de modelo é completa, isto é: $m_{i j}=1 \forall i, j$. Vale ressaltar que tais modelos possuem algumas propriedades especiais (ver apêndice A). Assim, podemos reescrever (2.2) na forma compacta

$$
\psi\left(x_{\bullet}, \beta\right)=\beta \times x_{\bullet}
$$

Campbell e Mosimann (1987) sugeriram um modelo de regressão fazendo os parâmetros $\lambda_{1}\left(x_{\bullet}\right), \lambda_{2}\left(x_{\bullet}\right), \ldots, \lambda_{D}\left(x_{\bullet}\right)$ dependerem polinomialmente da covariável $z_{\bullet}$ (supondo $z_{\bullet} \in \mathbb{R}$ ), isto é: $x_{\bullet}=\left(1, z_{\bullet}, \ldots, z_{\bullet}^{k-1}\right)$.

Devido às restrições sobre os parâmetros da distribuição de Dirichlet (devem ser todos positivos), devemos considerar apenas parâmetros $\beta_{i j}$ e funções $f_{1}, f_{2}, \ldots, f_{k}$ tais que $\psi\left(x_{\bullet} \mid \beta\right)>0$ para todo valor da covariável estendida $x_{\bullet}$. Sendo $x_{\bullet}$ uma variável contínua, essa condição é inviável de ser verificada na prática para uma dada matriz $\beta$. Assim, vamos impor somente que

$$
\psi\left(x_{j \bullet}, \beta\right)>0 \forall j \in\{1,2, \ldots, n\}
$$

Espera-se $^{1}$, assim, que $\psi\left(x_{j} \mid \beta\right)$ seja positivo para todo valor da covariável extendida $x \bullet$ (embora

\footnotetext{
${ }^{1}$ A condição $(2.5)$ garante que $\beta \times x_{\bullet}>0$ sempre que $x_{\bullet}$ for uma combinação linear de $\left(x_{1} \bullet\right),\left(x_{2} \bullet\right), \ldots,\left(x_{n} \bullet\right)$ com coeficientes positivos.
} 
isso não seja uma garantia).

Estimados os parâmetros $\beta$, podemos prever o valor do dado composicional referente ao valor $x_{0}$ • da covariável $x_{\bullet}$ tomando-se como estimativa o valor esperado da distribuição de Dirichlet com parâmetros $\lambda_{1}\left(x_{0 \bullet}\right), \lambda_{2}\left(x_{0 \bullet}\right), \ldots, \lambda_{D}\left(x_{0} \bullet\right)$, isto é:

$$
\operatorname{Prev}\left(x_{0} \bullet\right)=E\left(y\left(x_{0} \bullet\right)\right), \quad y\left(x_{0} \bullet\right) \sim \operatorname{Dir}\left(\lambda_{1}\left(x_{0} \bullet\right), \lambda_{2}\left(x_{0 \bullet}\right), \ldots, \lambda_{D}\left(x_{0} \bullet\right)\right)
$$

Pelo Teorema dos momentos, ver Pereira e Stern (2008), obtemos:

$$
\operatorname{Prev}\left(x_{0 \bullet}\right)=\left(\frac{\lambda_{1}\left(x_{0} \bullet\right.}{\Lambda\left(x_{0 \bullet}\right)}, \frac{\lambda_{2}\left(x_{0}\right)}{\Lambda\left(x_{0 \bullet \bullet}\right)}, \ldots, \frac{\lambda_{D}\left(x_{0} \bullet\right.}{\Lambda\left(x_{0 \bullet}\right)}\right), \quad \Lambda\left(x_{0 \bullet}\right)=\sum_{j=1}^{D} \lambda_{j}\left(x_{0 \bullet}\right)
$$

\subsection{Estimação de parâmetros}

Em seu estudo sobre o trabalho de Campbell e Mosimann, Hijazi e Jernigan (2009) propõem estimar os parâmetros $\beta_{i j}$ pela maximização da função de verossimilhança:

$$
L\left(\beta \mid Y_{1}, Y_{2}, \ldots Y_{n}\right)=\prod_{l=1}^{n}\left[\Gamma\left(\Lambda\left(x_{l \bullet}\right)\right) \prod_{i=1}^{D} \frac{\left.y_{l i}^{\lambda_{i}\left(x_{l}\right)}\right)-1}{\Gamma\left(\lambda_{i}\left(x_{l \bullet}\right)\right)}\right]
$$

Assim,

$$
\begin{gathered}
\log L=\sum_{l=1}^{n}\left[\log \Gamma\left(\Lambda\left(x_{l \bullet}\right)\right)+\sum_{i=1}^{D}\left(\lambda_{i}\left(x_{l \bullet}\right)-1\right) * \log y_{l i}-\log \Gamma\left(\lambda_{i}\left(x_{l \bullet}\right)\right)\right] \\
\frac{\partial \log L}{\partial \beta_{i j}}=\sum_{l=1}^{n}\left[\operatorname{diGamma}\left(\sum_{p=1}^{D} \lambda_{p}\left(x_{l \bullet}\right)\right)-\operatorname{diGamma}\left(\lambda_{i}\left(x_{l \bullet}\right)\right)+\log y_{l i}\right] * x_{l j} \\
\frac{\partial \log L}{\partial \beta_{a b} \partial \beta_{i j}}=\sum_{l=1}^{n}\left[\operatorname{triGamma}\left(\sum_{p=1}^{D} \lambda_{p}\left(x_{l \bullet}\right)\right)-\delta_{a i} * \operatorname{triGamma}\left(\lambda_{i}\left(x_{l \bullet}\right)\right)\right] * x_{l j} x_{l b}
\end{gathered}
$$

onde $\operatorname{diGamma}(u)=\frac{\partial \log \Gamma}{\partial u}(u), \quad \operatorname{triGamma}(u)=\frac{\partial^{2} \log \Gamma}{\partial u^{2}}(u)$ e $\delta_{a i}=\left\{\begin{array}{ll}1, & \text { se } a=i \\ 0, & \text { caso contrário }\end{array}\right.$.

Evidentemente, a complexidade da função $\nabla \log L$ não permite encontrar a solução da equação $\nabla \log L=0$ explicitamente. Hijazi e Jernigan propuseram um algoritmo para selecionar um valor inicial para algum método iterativo de maximização da função $\log L$, condicionado a (2.5). 


\subsubsection{Algoritmo de Hijazi-Jernigan para seleção de valores iniciais}

1. Extrair $r$ amostras $A_{1}, A_{2}, \ldots, A_{r}$ (todas de tamanho $m, m<n$ ) do conjunto $\left\{Z_{1}, Z_{2}, \ldots Z_{n}\right\}$, $Z_{j}=\left(x_{j \bullet}, y_{j \bullet}\right), j=1,2, \ldots, n$.

2. Para cada amostra $A_{i}=\left\{Z_{i_{1}}, Z_{i_{2}}, \ldots Z_{i_{m}}\right\}$, ajuste $\left\{y_{i_{1}} \bullet, y_{i_{2}} \bullet, \ldots, y_{i_{m}} \bullet\right\}$ por uma Dirichlet (pelo método da máxima verossimilhança, via algum algoritmo iterativo de maximização) com parâmetros $\widehat{\lambda_{i 1}}, \widehat{\lambda_{i 2}}, \ldots, \widehat{\lambda_{i D}}$, utilizando as estimativas obtidas pelo método dos momentos (ver Pereira e Stern (2008)) como ponto inicial. Calcule a média amostral da covariavel $z_{\bullet}$ em cada amostra, isto é: $\overline{z_{i \bullet}}=\frac{1}{m} \sum_{j=1}^{m} z_{i_{j}}$ e calcule os valores correspondentes da variável extendida $\overline{x_{i \bullet}}$.

Obtem-se, assim, as matrizes:

$$
\left[\begin{array}{cccc}
\widehat{\lambda_{11}} & \widehat{\lambda_{12}} & \ldots & \widehat{\lambda_{1 D}} \\
\widehat{\lambda_{21}} & \widehat{\lambda_{22}} & \ldots & \widehat{\lambda_{2 D}} \\
\vdots & \vdots & & \vdots \\
\widehat{\lambda_{r 1}} & \widehat{\lambda_{r 2}} & \ldots & \widehat{\lambda_{r D}}
\end{array}\right] \quad, \quad\left[\begin{array}{c}
\overline{x_{1}} \\
\overline{x_{2} \bullet} \\
\vdots \\
\overline{x_{r}}
\end{array}\right]
$$

3. Obter os parâmetros $\beta_{i j}$ que minimizam a norma euclidiana da diferença

$$
\left[\begin{array}{cccc}
\widehat{\lambda_{11}} & \widehat{\lambda_{21}} & \ldots & \widehat{\lambda_{r 1}} \\
\widehat{\lambda_{12}} & \widehat{\lambda_{22}} & \ldots & \widehat{\lambda_{r 2}} \\
\vdots & \vdots & & \vdots \\
\widehat{\lambda_{1 D}} & \widehat{\lambda_{2 D}} & \ldots & \widehat{\lambda_{r D}}
\end{array}\right]-\left[\begin{array}{cccc}
m_{11} \beta_{11} & m_{12} \beta_{12} & \ldots & m_{1 k} \beta_{1 k} \\
m_{21} \beta_{21} & m_{22} \beta_{22} & \ldots & m_{2 k} \beta_{1 k} \\
\vdots & \vdots & & \vdots \\
m_{D 1} \beta_{D 1} & m_{D 2} \beta_{D 2} & \ldots & m_{D k} \beta_{D k}
\end{array}\right] \times\left[\begin{array}{cccc}
\overline{x_{1} \bullet} & \overline{x_{2} \bullet} & \ldots & \overline{x_{r \bullet}}
\end{array}\right] .
$$

4. Utilizar os parâmetros obtidos como valores iniciais.

Vale ressaltar, porém, que o método iterativo de maximização da verossimilhança restrito à condição (2.5) requer que o ponto inicial também a satisfaça e não há garantias de que o algoritmo de Hijazi-Jernigan produza sempre pontos inciais com essa propriedade. Nós desenvolvemos um algoritmo mais eficiente para a obtenção de um ponto inicial.

\subsubsection{Novo algoritmo para seleção de valores iniciais}

$\mathbf{1}^{\circ}$ caso: $\mathrm{O}$ intercepto (função constante igual a 1 ) pertence ao conjunto das funções $\left\{f_{1}, f_{2}, \ldots, f_{k}\right\}$ definidas na Seção 1.1 .

1. Suponha que $f_{1}$ é a função constante igual a 1 , isto é $x_{\bullet}=\left(1, f_{2}\left(z_{\bullet}\right), \ldots\right)$ 
2. Introduza parâmetros artificiais $\beta_{j_{1} 1}, \beta_{j_{2} 1}, \ldots, \beta_{j_{l} 1}$, de modo a completar a primeira coluna da matriz de modelo, caso seja necessário. Para $D=4, k=3$ e

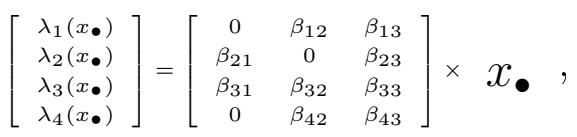

por exemplo, devemos completar com $\beta_{11}$ e $\beta_{4,1}$, isto é: $l=2, j_{1}=1$ e $j_{2}=4$.

3. Ajuste os dados observados $y_{1} \bullet, y_{2} \bullet, \ldots y_{n} \bullet$ por uma Dirichlet com parâmetros $\beta_{11}, \beta_{21}, \ldots, \beta_{D 1}$. O ponto incial $\beta^{*}=\left[\begin{array}{ccccc}\beta_{11} & \beta_{12}=0 & \beta_{13}=0 & \ldots & \beta_{1 k}=0 \\ \beta_{21} & \beta_{22}=0 & \beta_{23}=0 & \ldots & \beta_{1 k}=0 \\ \vdots & \vdots & & \vdots & \\ \beta_{D 1} & \beta_{D 2}=0 & \beta_{D 3}=0 & \ldots & \beta_{D k}=0\end{array}\right]$ certamente satisfaz $(2.5)$

4. Obtenha o ponto de máximo, por maximazação restrita à (2.5), da função

$\widetilde{L}=\log L\left(\beta \mid y_{1} \bullet, y_{2} \bullet, \ldots y_{n} \bullet\right)-M(\eta) \sum_{r=1}^{l}\left(\beta_{j_{r} 1}\right)^{2}$, utilizando o ponto inicial $\beta^{*}$, onde $M(\eta)$ é uma quantidade positiva que começa com um valor baixo e vai aumentando ao longo do número de iterações $\eta$.

5. Descarte os valores correspondentes aos parâmetros artificiais adicionados (os valores descartados deverão ser próximos de zero).

$\mathbf{2}^{\mathbf{o}}$ caso: $\mathrm{O}$ intercepto não pertence ao conjunto das funções $\left\{f_{1}, f_{2}, \ldots, f_{k}\right\}$.

1. Repita o $1^{\circ}$ caso adicionando a função constante igual a 1 à esse conjunto (note que, nesse caso, serão introduzidas D variáveis artificiais)

Observe que a função $\widetilde{L}$ penaliza os parâmetros eventualmente adicionados de tal forma que, ao fim do processo de maximização iterativo, os valores dos parâmetros adicionados deverão ser próximos a zero. Além disso, o fato de $\beta^{*}$ sempre satisfazer (2.5) garante um método mais estável de obtenção dos parâmetros de máxima verossimilhança.

\subsubsection{Resultados numéricos}

Para avaliar a eficiência dos métodos propostos para selecionar um valor inicial no modelo de Dirichlet linear, realizamos algumas simulações baseadas em modelos polinomiais de grau menor ou igual a $3\left(k=4, z_{\bullet} \in \mathbb{R}, x_{\bullet}=\left(1, z_{\bullet}, z_{\bullet}^{2}, z_{\bullet}^{3}\right)\right.$ com um conjunto de subamostras do conjunto de dados do Lago Ártico. Foram selecionadas (aleatoriamente) $\mathrm{m}=1000$ subamostras com $50 \%$ (n=20) e $70 \%(\mathrm{n}=27)$ dos pontos do conjunto de dados original. Para cada subamostra, foram definidas, por processos de Bernoulli, três Matrizes de modelo com porcentagens de 1's próximas a $p=0.33, p=0.5$ 
e $p=0.66$. Para evitar modelos inconsistentes, também foi imposto que tais matrizes deveriam apresentar ao menos uma entrada não nula por linha. Duas medidas de eficiência foram analisadas: (1) A porcentagen dos casos em que os algoritmos não foram capazes de encontrar pontos iniciais viáveis (limitado a trinta tentativas ${ }^{2}$ por subamostra); (2) O tempo médio de processamento de cada método. Os resultados são mostrados na Figura 2.1. O tamanho das subamostras (20 ou 27) pareceu não alterar a performance e, assim, os resultados são mostrados juntos.

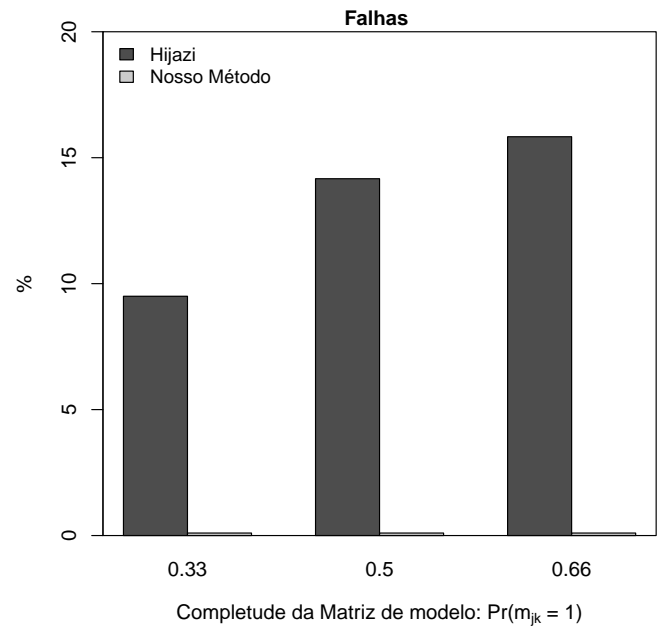

(a)

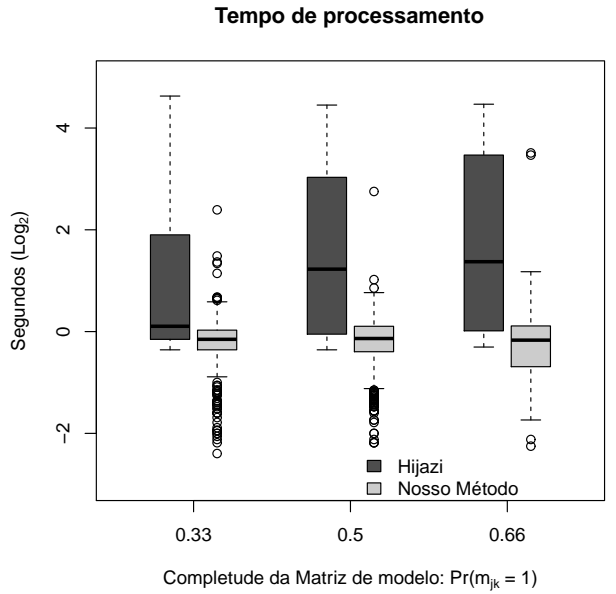

(b)

Figura 2.1: Comparativo entre os métodos de seleção de valores iniciais para o modelo de Dirichlet linear

O gráfico da esquerda mostra claramente uma maior estabilidade do nosso algoritmo: em todos os 6000 casos conseguimos um ponto inicial dentro da região viável. É claro que o tempo de processamento depende da forma como os algoritmos foram implementados. Com a nossa implementação, o nosso algoritmo mostrou também uma superioridade com relação ao outro, mostrada no gráfico da direita: a distribuição dos tempos nos três casos, para o nosso algoritmo, está mais concentrada em torno das medianas que, por sua vez, são inferiores às correspondentes produzidas pelo algoritmo de Hijazi-Jernigan.

\subsection{Eliminação das restrições sobre o espaço paramétrico}

Uma variante do modelo de regressão linear, proposto em Melo et al. (2009), consiste em escolher $D$ funções positivas $g_{j}: \mathbb{R} \longrightarrow \mathbb{R}^{+}, j=1,2, \ldots, D$ (injetoras e 3 vezes diferenciáveis) e aplicar a função $g_{j}$ à $j$-ésima componente do produto (2.4) . Em outras palavras, supor que a função

$$
\psi\left(x_{\bullet} \mid \beta\right),
$$

é da forma

\footnotetext{
${ }^{2}$ Note que apenas o método de Hijazi-Jernigan possui um procedimento envolvendo eventos aleatórios e, portanto, apenas este produz resultados diferentes para todos os parâmetros de maximização fixados.
} 


$$
\begin{aligned}
& \psi\left(x_{\bullet}, \beta\right)=\left[\begin{array}{c}
\lambda_{1}\left(x_{\bullet}\right) \\
\lambda_{2}\left(x_{\bullet}\right) \\
\vdots \\
\lambda_{D}\left(x_{\bullet}\right)
\end{array}\right]=\left[\begin{array}{c}
g_{1}\left(m_{11} \beta_{11} x_{1}+m_{12} \beta_{12} x_{2}+\ldots+m_{1 k} \beta_{1 k} x_{k}\right) \\
g_{2}\left(m_{21} \beta_{21} x_{1}+m_{22} \beta_{22} x_{2}+\ldots+m_{2 k} \beta_{1 k} x_{k}\right) \\
\vdots \\
g_{D}\left(m_{D 1} \beta_{D 1} x_{1}+m_{D 2} \beta_{D 2} x_{1}+\ldots+m_{D k} \beta_{D k} x_{k}\right)
\end{array}\right], \\
& x_{\bullet}=\left(x_{1}, \ldots, x_{k}\right) .
\end{aligned}
$$

Neste modelo, a condição (2.5) pode ser eliminada. Sob as hipóteses sobre as funções $g_{j}, j=$ $1,2, \ldots, D$ e sobre a matriz $X$ de $(1.1)$, os autores garantem a existência e unicidade do estimador de máxima verossimilhança, baseados em um resultado de Krätschmer (2007). Porém,o que Krätschmer mostra é que o conjunto de possíveis amostras tais que o a função de verossimilhança assume um máximo em dois ou mais pontos distintos possui medida de Lebesgue nula. Isso garante a unicidade do estimador de máxima verossimilhança com probabilidade 1, mas não garante a existência.

Naquele trabalho, os autores utilizam a função exponencial $\left(g_{j}(t)=e^{t} \forall j\right)$. Em algumas de nossas simulações com os dados do Lago Ártico, porém, o uso da função exponencial apresentou muita instabilidade. Uma alternativa é utilizar a função Arcotangente que, a menos de uma constante positiva maior do que $\frac{\pi}{2}$, também satisfaz a essas condições (mais comentários no Capítulo 4, Figura 4.2). 


\section{Capítulo 3}

\section{Outros modelos de regressão}

\subsection{Transformações $S_{D} \longmapsto \mathbb{R}^{D-1}$}

Segundo Aitchison (1982), uma das dificuldades iniciais para a regressão de dados composicionais é a escassez de famílias paramétricas de modelos probabilísticos definidos no $D$ - 1-Simplex (a mais conhecida é a Distribuição de Dirichlet). Aitchison propõe contruir novos modelos probabilísticos no Simplex utilizando distribuições conhecidas em $R^{d}(d=D-1)$ por meio de transformações bijetoras convenientes. As distribuições Normais-Logísticas, apresentadas em Aitchison e Shen (1980), por exemplo, são provenientes da transformação de variáveis com distribuição normal multivariada via transformação Logaritmo da razão (alr) :

$$
\left(y_{1}, y_{2}, \ldots, y_{D}\right) \in S_{D} \stackrel{a l r}{\longmapsto}\left(\log \left(y_{1} / y_{D}\right), \log \left(y_{2} / y_{D}\right), \ldots \log \left(y_{D-1} / y_{D}\right)\right) \in \mathbb{R}^{d}
$$

Essa nova classe de distribuições definidas no Simplex, porém, não é de serventia imediata para a aplicação do método descrito no Capítulo introdutório, pois "Embora existam os momentos de todas as ordens ... suas expressões integrais ${ }^{1}$ não são redutíveis a uma forma simples"Aitchison e Shen (1980). Aitchison, sugere transformar os dados do Simplex para $\mathbb{R}^{d}$, aplicar o método para os dados transformados, e retornar para o Simplex. Em um dos seus estudos de caso, ele se propõe a analisar como varia a composição do solo do Lago Ártico em função da profundidade $z$ (Aitchinson (1986)), supondo que o vetor

$$
\mu(x)=\left[\begin{array}{c}
\log \left(\frac{\text { sand }}{\text { clay }}\right)(z)=\beta_{11}+\beta_{12} z+\beta_{13} z^{2}+\beta_{14} \log (z) \\
\log \left(\frac{\text { silt }}{\text { clay }}\right)(z)=\beta_{21}+\beta_{22} z+\beta_{23} z^{2}+\beta_{24} \log (z)
\end{array}\right]=\beta \times x, \quad x=\left(1, z, z^{2}, \log (z)\right)
$$

representa o valor esperado de uma distribuição normal bivariada $N^{2}(\mu(z), \Sigma)$, onde $\Sigma$ independe de $z$. Aitchison sugere a estimação dos parâmetros $\beta_{i j}$ e $\Sigma$ por mínimos quadrados multivariado ou, sob a hipótese de normalidade, pela maximização da função de verossimilhança (Aitchinson (1986), pág. 160).

\footnotetext{
${ }^{1}$ Em particular, a expressão correspondente ao valor esperado
} 
No caso geral, supondo que

$$
\operatorname{alr}\left(y_{\bullet}\right) \mid x_{\bullet} \sim N^{d}\left(\mu\left(x_{\bullet}\right), \Sigma\right),
$$

a função de verossimilhança é dada por: $L\left(\beta i j, 1 \leq i \leq d, 1 \leq j \leq k, \Sigma \mid \operatorname{alr}\left(y_{1} \bullet\right), \ldots \operatorname{alr}\left(y_{n} \bullet\right)\right)=$

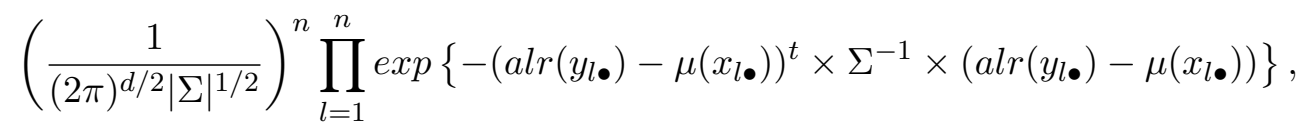

onde $\mu(x)=\psi(x, \beta)$ possui a mesma forma de (2.2).

Vale ressaltar que:

- Para modelos com matriz de modelo completa, os estimadores (para $\beta$ ) de máxima verossimilhança e de mínimos quadrados, que minimiza

$$
\operatorname{Res}^{2}(\beta)=\sum_{i=1}^{n}\left\|\left(\operatorname{alr}\left(y_{i \bullet}\right)-\mu\left(x_{i \bullet}\right)\right)\right\|^{2}
$$

são idênticos e podem ser obtidos por meio da resolução de um sistema linear (ver apêndice A).

- No caso de ajuste total (onde o resíduo quadrático (3.5) é igual a zero) a função de verossimilhana (3.4), calculada nos parâmetros $\beta_{i j}$ de ajuste total, depende de $\Sigma$ apenas pelo inverso do seu determinante, o qual pode aproximar-se indefinidamente de zero ${ }^{2}$. Isso mostra que, nesse caso, a função de verossimilhança não é limitada em $\Sigma$ e, portanto, a estimação de $\Sigma$ por máxima verossimilhança pode, eventualmente, não fazer sentido. Determinar quais casos são patológicos, porém, parece ser uma tarefa complexa.

\subsubsection{Abrangência do método}

Ressaltamos que o desenvolvimento descrito acima pode ser feito para qualquer transformação de $S_{D}$ para $\mathbb{R}^{d}$. Além da transformação alr, as seguintes transformações são apresentadas em Aitchison (1982).

\footnotetext{
${ }^{2}$ Um exemplo de uma sequência de matrizes positivas-definidas com essa propriedade é $\left(\frac{1}{k} I\right)_{k \in \mathbb{N}}$, onde $I$ denota a matriz identidade.
} 


\begin{tabular}{|c|c|}
\hline Nome & Especificação \\
\hline Multiplicativa $\left(M_{d}\right)$ & $v_{i}=\log \frac{y_{i}}{1-\sum_{j=1}^{i} y_{j}}, i=1,2, \ldots, d$ \\
\hline Híbrida $\left(H_{d}\right)$ & $v_{1}=\log \frac{y_{1}}{1-y_{1}}, \quad v_{i}=\log \frac{y_{i}}{\left(1-\sum_{j=1}^{i-1} y_{j}\right)\left(1-\sum_{j=1}^{i} y_{j}\right)}, i=2, \ldots, d$ \\
\hline
\end{tabular}

Tabela 3.1: Transformações logísticas elementares $y \in S_{D} \longmapsto v \in \mathbb{R}^{d}$

Outra transformação pode ser obtida por meio da transformação $c l r$ (centered log-ratio):

$$
y=\left(y_{1}, y_{2}, \ldots, y_{D}\right) \in S_{D} \stackrel{c l r}{\longmapsto}\left(\log \left(y_{1} / g(y)\right), \log \left(y_{2} / g(y)\right), \ldots \log \left(y_{D} / g(y)\right)\right) \in V
$$

apresentada em Aitchison et al. (2000), entre o $D$ - 1-Simplex e o hiperplano $V$, paralelo a ele, onde $g(y)$ é a média geométrica da composição $y$. No entanto, o contra-domínio da transformação $c l r$, não é o espaço euclidiano $\mathbb{R}^{d}$ mas sim um subespaço de $\mathbb{R}^{D}$ isomorfo a ele (os vetores transformados possuem, ainda, $D$ componentes). Para obtermos vetores em $\mathbb{R}^{d}$, precisamos compor a função $c l r$ com algum isomorfismo linear $T$ entre $V$ e $R^{d}$ como, por exemplo, a função que retorna as coordenadas de um elemento de $V$ com relação à uma certa base $B$. Uma possível escolha para $B$ é:

$$
B=\left\{\frac{v_{1}}{\left\|v_{1}\right\|}, \frac{v_{2}}{\left\|v_{2}\right\|}, \ldots, \frac{v_{d}}{\left\|v_{d}\right\|}\right\}, v_{j}=(1, \ldots, 1, \underbrace{-j}_{\text {posição }}, 0, \ldots, 0)
$$

Porém, simulações com os dados do Lago Ártico geraram as mesmas funções de previsão, trocando-se a transformação alr pela transformação $T \circ \mathrm{clr}$ (para o isomorfismo $T$ relativo à base (3.7)). Determinar as condições sob as quais transformações distintas levam a resultados idênticos caracteriza, portanto, uma questão de fundamental importância para determinar a abrangência do método descrito na seção anterior. Aitchison dá o primeiro avanço nessa direção, ao mostrar que a função de previsão (para a transformação alr) é invariante sobre permutações das componentes das composições (ver Aitchinson (1986), pág. 161). Nós obtivemos uma resposta geral para essa questão:

Definição: Duas transformações $T_{1}, T_{2}: S_{D} \longrightarrow \mathbb{R}^{d}$ são equivalentes se $T_{1}\left(y_{\bullet}\right) \mid x_{\bullet} \sim N\left(\mu_{1}\left(x_{\bullet}\right)=\right.$ $\left.\beta_{1} \times x_{\bullet}, \Sigma_{1}\right)$ sempre que $T_{2}\left(y_{\bullet}\right) \mid x_{\bullet} \sim N\left(\mu_{2}\left(x_{\bullet}\right)=\beta_{2} \times x_{\bullet} \Sigma_{2}\right)$ e vice-versa.

Teorema 1. Duas transformaçôes $T_{1}, T_{2}: S_{D} \longrightarrow R^{d}$ são equivalentes se e somente se $T_{1} \circ T_{2}^{-1}$ : $\mathbb{R}^{d} \longrightarrow \mathbb{R}^{d}$ é uma transformaçẫo linear. Nessas condiçôes, as funções de previsão Prev $v_{T_{1}}\left(x_{\bullet}\right)=$ $T_{1}^{-1}\left(\widehat{\beta_{1}} \times x_{\bullet}\right)$ and $\operatorname{Prev}_{T_{2}}\left(x_{\bullet}\right)=T_{2}^{-1}\left(\widehat{\beta_{2}} \times x_{\bullet}\right)$, dadas pelas estimativas de máxima verossimilhança $\widehat{\beta_{1}}$ e $\widehat{\beta_{2}}$, são idênticas (para modelos com matriz de modelo completa). 


\section{Prova:}

Suponha que $T_{1} \circ T_{2}^{-1}: U \longmapsto M \times U$ é linear ( $M$ denota a matriz da transformação $T_{1} \circ T_{2}^{-1}$ com relação à base canônica de $\mathbb{R}^{d}$ ).

É um fato conhecido sobre a distribuição Normal, que

$$
T_{2}\left(y_{\bullet}\right)|x_{\bullet} \sim N\left(\beta_{2} \times x_{\bullet}, \Sigma_{2}\right) \Longleftrightarrow \underbrace{M \times T_{2}\left(x_{\bullet}\right)}_{T_{1}\left(x_{\bullet}\right)}| x_{\bullet} \sim N\left(M \times \beta_{2} \times x_{\bullet} M \times \Sigma_{2} \times M^{t}\right) .
$$

Por (A.5), temos que

$$
\begin{aligned}
& \sum_{l=1}^{n} x_{l j}\left(T_{1}\left(y_{l \bullet}\right)-\widehat{\beta_{1}} \times x_{l \bullet}=0, j=1,2, \ldots, k\right. \\
& \sum_{l=1}^{n} x_{l j}\left(T_{2}\left(y_{l \bullet}\right)-\widehat{\beta_{2}} \times x_{l \bullet}=0, j=1,2, \ldots, k .\right.
\end{aligned}
$$

Substituindo $\quad T_{1}\left(y_{l}\right)=T_{1} \circ T_{2}^{-1}\left(T_{2}\left(y_{l} \bullet\right)\right)=M \times T_{2}\left(y_{l} \bullet\right)$ em (3.8), obtemos

$$
\sum_{l=1}^{n} x_{l j}\left(T_{2}\left(y_{l \bullet}\right)-M^{-1} \times \widehat{\beta}_{1} \times x_{l \bullet}=0, j=1,2, \ldots, k .\right.
$$

Comparando (3.9) e (3.10), devemos ${ }^{3}$ ter $\widehat{\beta_{1}}=M \times \widehat{\beta_{2}}$ e, então,

$$
\operatorname{Prev}_{\left[T_{1}\right]}\left(x_{\bullet}\right)=T_{1}^{-1}\left(M \times \widehat{\beta}_{2} \times x_{\bullet}\right)=T_{2}^{-1}\left(\widehat{\beta}_{2} \times x_{\bullet}\right)=\operatorname{Prev}_{\left[T_{2}\right]}\left(x_{\bullet}\right) .
$$

Por outro lado, suponha que $T_{1}$ e $T_{2}$ são equivalentes e seja $\Phi=T_{1} \circ T_{2}^{-1}$.

Tome $k=d$ e $f_{1}, f_{2}, \ldots, f_{d}$, definidas no capítulo introdutório, tais que $x_{\bullet}=z_{\bullet} \forall z_{\bullet} \in \Omega$.

Por hipótese, para $w\left(x_{\bullet}\right)=T_{1}\left(y_{\bullet}\right) \mid x_{\bullet} \sim N\left(x_{\bullet}, \Sigma_{1}\right)\left(\beta_{1}=\right.$ matriz identidade), devemos ter

$$
\zeta=\zeta\left(x_{\bullet}\right)=T_{2}\left(y_{\bullet}\right) \mid x_{\bullet} \sim N\left(\beta_{2} \times x_{\bullet}, \Sigma_{2}\right),
$$

para certas matrizes $\beta_{2}, \Sigma_{2}(\forall x \bullet \in \Omega)$.

\footnotetext{
${ }^{3}$ Pois $M \times \widehat{\beta_{2}}$ também satisfaz (A.5).
} 
Além disso, se $w\left(x_{\bullet}\right)$ possui f.d.p. (função densidade de probabilidade) $f\left(w \mid x_{\bullet}\right)$, então $\zeta\left(x_{\bullet}\right)$ possui f.d.p. $h\left(\zeta \mid x_{\bullet}\right)=f\left(\Phi(\zeta) \mid x_{\bullet}\right)\left|\frac{\partial \Phi}{\partial \zeta}\right|$.

Por $(3.11), h\left(\zeta \mid x_{\bullet}\right)$ é simétrica com relação a $\zeta$ e $\beta_{2} \times x_{\bullet}$. Logo, seu gradiente (como função de $\left.x_{\bullet}\right)$ deve se anular em $x_{\bullet}^{*}=\beta_{2}^{-1} \times \zeta$. Portanto,

$$
0=\nabla h\left(x_{\bullet}^{*} \mid \zeta\right) \times \beta_{2}=\nabla f\left(x_{\bullet}^{*} \mid \Phi(\zeta)\right) \times \underbrace{\left|\frac{\partial \Phi}{\partial \zeta}(\zeta)\right|}_{\neq 0} .
$$

Segue que $x_{\bullet}^{*}=\Phi(\zeta)$, isto é: $\Phi(\zeta)=\beta_{2}^{-1} \times \zeta\left(\forall \zeta \in \mathbb{R}^{d}\right)$

Corolário 1. 1. alr e alr $\circ P$ são equivalentes para qualquer permutação $P$ das componentes das composições.

2. $T \circ$ clr e alr são equivalentes para todo isomorfismo linear $T: V \longrightarrow \mathbb{R}^{d}$

3. As Transformaçôes logísticas Multiplicativa $\left(M_{d}\right)$ e Híbrida $\left(H_{d}\right)$ são equivalentes somente para $D \leq 3$.

Prova:

1. Sejam $T_{1}=a l r \circ P$ e $T_{2}=a l r$. Sem perda de generalidade, vamos supor que $P$ é uma transposição, isto é: $P\left(y_{1}, \ldots, y_{i}, \ldots, y_{j}, \ldots, y_{D}\right)=\left(y_{1}, \ldots, y_{j}, \ldots, y_{i}, \ldots, y_{D}\right)$.

Para $u=T_{2}(y) \in R^{d}$, temos:

$1^{o}$ caso: $i<j<D$.

$$
\begin{aligned}
T_{1}\left(T_{2}^{-1}(u)\right) & =T_{1}(y) \\
& =\operatorname{alr}\left(\left(y_{1}, \ldots, y_{j}, \ldots, y_{i}, \ldots, y_{D}\right)\right) \\
& =\left(\log \left(y_{1} / y_{D}\right), \ldots \log \left(y_{j} / y_{D}\right), \ldots, \log \left(y_{i} / y_{D}\right), \ldots \log \left(y_{D-1} / y_{D}\right)\right) \\
& =\left(u_{1}, \ldots, u_{j}, \ldots, u_{i}, \ldots, u_{d}\right)
\end{aligned}
$$

$2^{\circ}$ caso: $i<j=D$.

$$
\begin{aligned}
T_{1}\left(T_{2}^{-1}(u)\right) & =T_{1}(y) \\
& =\operatorname{alr}\left(\left(y_{1}, \ldots, y_{j}, \ldots, y_{D-1}, y_{i}\right)\right) \\
& =\left(\log \left(y_{1} / y_{i}\right), \ldots, \log \left(y_{j} / y_{i}\right), \ldots, \log \left(y_{D-1} / y_{i}\right)\right) \\
& =\left(u_{1}-u_{i}, \ldots, u_{j}-u_{i}, \ldots, u_{d}-u_{i}\right)
\end{aligned}
$$


2. Para $u=\operatorname{alr}(Y)$, temos

$$
T(\operatorname{clr}(Y))=T\left(\frac{D-1}{D} u_{1}-\frac{1}{D} \sum_{j \neq 1} u_{j}, \ldots, \frac{D-1}{D} u_{d}-\frac{1}{D} \sum_{j \neq d} u_{j},-\frac{1}{D} \sum_{j=1}^{d} u_{j}\right)
$$

3. - Para $D=2, M_{d}(Y)=H_{d}(Y) \forall Y \in S_{D}$.

- Para $D=3$ e $M_{d}(Y)=u$, temos $H_{d}(Y)=\left(u_{1}, u_{2}-u_{1}\right)$.

- Para $D>3, M_{d}(Y)=u$ e $H_{d}(Y)=w$, temos que $w_{3}=u_{3}+u_{2}-\log \left(y_{2}\right)$, e $\log \left(y_{2}\right)$ não pode ser expressa como combinação linear de $u_{1}, u_{2}, \ldots, u_{d}$.

\subsection{Coordenadas esféricas}

Wang et al. (2007) sugeriram um modelo de regressão baseado em outra parametrização do Simplex por coordenadas esféricas ${ }^{4}$ :

$$
\begin{aligned}
& \left.\left.\left(\alpha_{1}, \alpha_{2}, \ldots, \alpha_{d}\right) \in(] 0, \frac{\pi}{2}\right]\right)^{d} \longmapsto y \in \overline{S_{D}} \\
& \gamma_{1}=\prod_{j=1}^{d} \sin \left(\alpha_{j}\right), \\
& \gamma_{i}=\cos \left(\alpha_{i-1}\right) \prod_{j=i}^{d} \operatorname{sen}\left(\alpha_{j}\right), \quad i=2,3, \ldots, D \\
& y_{j}=\gamma_{j}^{2}, \quad j=1,2, \ldots, D .
\end{aligned}
$$

Nesse modelo, os dados transformados, correspondentes às coordenadas angulares $\alpha_{1}, \alpha_{2}, \ldots, \alpha_{d}$, são modelados diretamente em função das covariáveis por algum método de inferência. Segundo os autores, esse modelo apresenta uma vantagem sobre o método de Aitchison, já que, como não há divisões, o modelo comporta dados composicionais com componentes eventualmente nulas. Infelizmente, porém, o problema de lidar com componentes nulas (o qual nos abstemos nesta apresentação) não nos parece ter uma solução tão simples.

Segundo os autores (que se limitam ao uso de covariáveis unidimensionais, mais precisamente o tempo), o modelo consiste em construir $d$ modelos, um para cada coordenada angular:

$$
\theta_{j}(t)=h_{j}(t)+\epsilon_{j}(t), \quad j=1,2, \ldots, d,
$$

onde $h_{j}(t), j=1,2, \ldots, d$ são as funções que descrevem os modelos e $\epsilon_{j}(t)$ são os erros observados.

\footnotetext{
${ }^{4} \mathrm{O}$ qual suporta componentes nulas nas composições.
} 
Naquele trabalho, os autores utilizam funções polinomiais para as funções $h_{j}, j=1,2, \ldots, d$. Mais em geral, podemos supor que tais funções são combinações lineares de funções de livre escolha, como assumido nos Capítulos anteriores, isto é:

$$
\left[\begin{array}{c}
h_{1}(t) \\
h_{2}(t) \\
\vdots \\
h_{d}(t)
\end{array}\right]=\left[\begin{array}{cccc}
m_{11} * \beta_{11} & m_{12} * \beta_{12} & \ldots & m_{1 k} * \beta_{1 k} \\
m_{21} * \beta_{21} & m_{22} * \beta_{22} & \ldots & m_{2 k} * \beta_{1 k} \\
\vdots & \vdots & & \vdots \\
m_{D 1} * \beta_{d 1} & m_{d 2} * \beta_{d 2} & \ldots & m_{d k} * \beta_{d k}
\end{array}\right] \times\left[\begin{array}{c}
f_{1}(t) \\
f_{2}(t) \\
\vdots \\
f_{k}(t)
\end{array}\right],
$$

onde $f_{1}(t), f_{2}(t), \ldots, f_{k}(t)$ e $M_{\text {mod }}=\left(m_{i j}\right)$ são definidas nas Seções 1.1 e 2.2, respectivamente. Sob tais hipóteses, podemos listar alguns inconvenientes na implementação e aplicação desse modelo de regressão:

1. Uma primeira dificuldade diz respeito ao método de inferência utilizado para a estimação dos coeficientes $\beta_{s}$. A escolha mais natural, certamente, é a estimação por mínimos quadrados. Porém, assim como no modelo de Dirichlet linear, o processo de otimização deve ser condicionado, já que os ângulos devem sempre pertencer ao intervalo $[0, \pi / 2]$. Por outro lado, a função a ser minimizada (a soma dos quadrados dos resíduos) é uma forma quadrática positiva definida e, portanto, assume um único ponto de mínimo (o único que anula o gradiente). Consequentemente, se um tal ponto de mínimo não se encontra na região viável, qualquer algoritmo de maximização condicionada resultará em um ponto da fronteira. Em outras palavras, existirão índices $i$ e $j$ para os quais teremos $h_{j}\left(t_{i}\right)=0$ ou $h_{j}\left(t_{i}\right)=\pi / 2$ e, portanto, há grande chances de que $h_{j}(t)<0$ ou $h_{j}(t)>\pi / 2$, para $t$ próximo à $t_{i}$, o que tornaria o modelo inutilizável.

2. Ironicamente, esse inconveniente se torna ainda mais provável nos casos para os quais o modelo foi desenvolvido, isto é, nas aplicações onde o conjunto de dados possui composições com algumas componentes nulas. Pois, para qualquer método de inferência cujo objetivo seja a aproximação das funções $h_{1}, h_{2}, \ldots, h_{d}$ aos dados observados, componentes nulas gerarão funções assumindo valores próximos à região da fronteira.

Não fomos capazes de reproduzir os resultados apresentados pelos autores na análise de seus conjuntos de dados (vale ressaltar que o método de inferência utilizado não foi explicitado).

O primeiro conjunto de dados, constituído por doze composicões com quatro componentes cada, foi gerado para simulações (a covariável assumindo os valores $1,2, \ldots, 12$ ). O segundo, constituído por doze composicões com três componentes cada, é referente à variação das componentes da economia chinesa (setor primário, secundário e terciário) entre os anos de 1990 e 2001. Considerando modelos polinomiais, os autores expôem as seguintes estimativas

$$
\begin{aligned}
& \theta_{1}^{*}(t)=1.4289+0.0304 t^{2}-0.0009 t^{3} \\
& \theta_{2}^{*}(t)=1.6346-0.1263 t \\
& \theta_{3}^{*}(t)=1.4546+0.1149 t-0.0317 t^{2}+0.0013 t^{3}
\end{aligned} \quad \text { e } \quad \begin{aligned}
\theta_{1}^{\prime}(t) & =1.0572-0.0161 t+0.0007 t^{2} \\
\theta_{2}^{\prime}(t) & =1.1572-0.0214 t+0.0008 t^{2}
\end{aligned} .
$$


Nós obtivemos as seguintes estimativas de mínimos quadrados (por maximização irrestrita):

$$
\begin{aligned}
& \theta_{1}^{a}(t)=(9.864 e-1)-(3.627 e-2) t^{2}+(2.753 e-3) t^{3} \\
& \theta_{2}^{a}(t)=(1.635 e+0)-(1.264 e-1) t \\
& \theta_{3}^{a}(t)=(1.486 e+0)+(1.012 e-1) t-(2.983 e-2) t^{2}+(1.221 e-3) t^{3} \\
& \theta_{1}^{b}(t)=(2.873 e+3)-(2.871 e+0) t+(7.178 e-4) t^{2} \\
& \theta_{2}^{b}(t)=(3.077 e+3)-(3.071 e+0) t+(7.666 e-4) t^{2} .
\end{aligned}
$$

Para o primeiro conjuntos de dados, a estimativa de mínimos quadrados não encontra-se dentro da região viável $\left(\theta_{3}^{a}(2)=1.579>\pi / 2\right)$.

\subsection{Outras transformações no Simplex}

Motivados pelo método geral proposto na Seção 3.1.1, fomos à procura de mais transformações entre o Simplex e o Espaço euclidiano. De forma independente ${ }^{5}$, encontramos uma série de outras transformações baseadas em coordenadas esféricas generalizadas.

Ao contrário das transformações da tabela 3.1, a transformação alr possui uma fácil interpretação geométrica, como mostrado na figura 3.1.

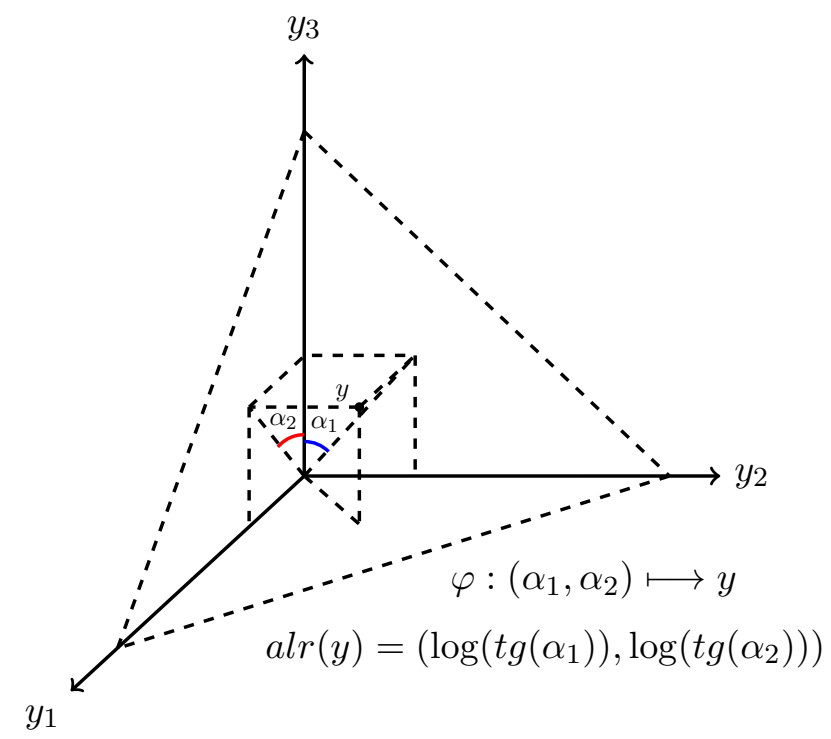

Figura 3.1: Interpretação geométrica da transformação alr

\footnotetext{
${ }^{5} \mathrm{O}$ trabalho de Wang só foi conhecido posteriormente.
} 
Como podemos observar, essa transformação é baseada na parametrização $\varphi$, do Simplex, em termos das coordenadas angulares periféricas $\alpha_{1}$ e $\alpha_{2}$. Mais transformações são obtidas ao considerarmos a parametrização do Simplex por coordenadas angulares centrais (coordenadas esféricas), como exemplificado na figura 3.2 , para $D=3$.

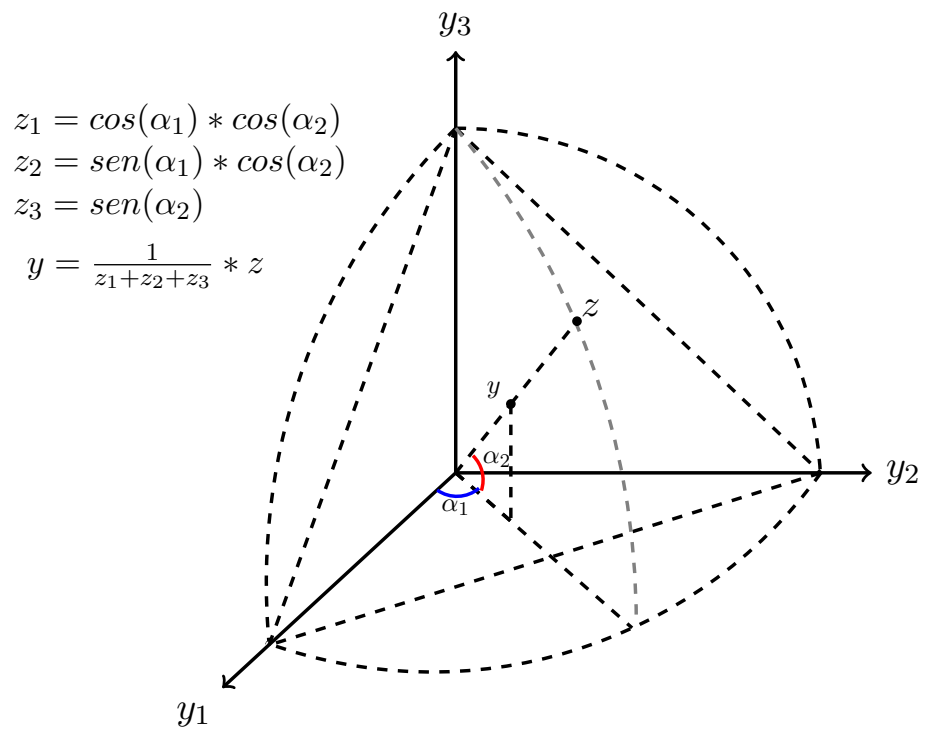

Figura 3.2: Parametrização do 3-Simplex por coordenadas esféricas

Essa parametrização pode ser extendida para $D>3$, utilizando coordenadas esféricas generalizadas:

$$
\begin{aligned}
& \varphi:\left(\alpha_{1}, \alpha_{2}, \ldots, \alpha_{d}\right) \in(] 0, \frac{\pi}{2}[)^{d} \longmapsto y \in S_{D} \\
& z_{1}=\prod_{j=1}^{d} \cos \left(\alpha_{j}\right), \\
& z_{i}=\operatorname{sen}\left(\alpha_{i-1}\right) \prod_{j=i}^{d} \cos \left(\alpha_{j}\right), \quad i=2,3, \ldots, D \\
& y \quad=z / \sum_{j=1}^{d} z_{j}
\end{aligned}
$$

e a sua inversa

$$
z=y /\|y\| \quad \alpha_{i}=t g^{-1}\left(\frac{z_{i+1}}{\sqrt{\sum_{j=1}^{i} z_{j}^{2}}}\right)
$$


Obtendo-se as cordenadas angulares $\alpha_{1}, \alpha_{2}, \ldots, \alpha_{d}$, podemos compor novas transformações entre o Simplex e o Espaço Euclidiano, aplicando-se as funções tangente e/ou logaritmo (ou outras funções adequadas). Note que há relação biunívoca entre os modelos baseados em coordenadas angulares periféricas e centrais. A transformação correspondente à Logaritmo da razão, em coordenadas esféricas, é a transformação Log-esférica, dada por:

$$
\begin{gathered}
y \in S_{D} \stackrel{\log E s f}{\longmapsto}\left(\log \left(\operatorname{tg}\left(\alpha_{1}\right)\right), \log \left(\operatorname{tg}\left(\alpha_{2}\right)\right), \ldots, \log \left(\operatorname{tg}\left(\alpha_{d}\right)\right)\right) \in \mathbb{R}^{d}, \\
z=y /\|y\|, \quad \alpha_{i}=t g^{-1}\left(\frac{z_{i+1}}{\sqrt{\sum_{j=1}^{i} z_{j}^{2}}}\right) .
\end{gathered}
$$

Aplicando-se a função tangente diretamente, obtemos uma nova classe de transformações:

Definição: A transformação Tangente-esférica com parâmetros $\left.h_{1}, h_{2}, \ldots, h_{d}:\right] 0, \frac{\pi}{2}[\longrightarrow]-\frac{\pi}{2}, \frac{\pi}{2}[$ é definida por

$$
y \in S_{D} \stackrel{\text { TgEsf }}{\longmapsto}\left(t g\left(h_{1}\left(\alpha_{1}\right)\right), \operatorname{tg}\left(h_{2}\left(\alpha_{2}\right)\right), \ldots, t g\left(h_{d}\left(\alpha_{d}\right)\right)\right) \in \mathbb{R}^{d}
$$

onde $\alpha_{1}, \alpha_{2}, \ldots, \alpha_{d}$ são dados em (3.20).

A transformação correspondente à tangente-esférica com parâmetros lineares $\left(h_{j}(x)=2 x-\right.$ $\pi / 2, \forall j)$, em coordenadas angulares periféricas, é:

$$
y \in S_{D} \stackrel{\text { TgRatio }}{\longmapsto} z \in \mathbb{R}^{d}, \quad z_{j}=\operatorname{tg}\left(2 * \operatorname{arctg}\left(\frac{y_{j}}{y_{D}}\right)-\frac{p i}{2}\right), j=1,2, \ldots, d
$$

Utilizando a relação $\operatorname{tg}(2 x-\pi / 2)=\frac{1}{2}\left(\operatorname{tg}(x)-\frac{1}{t g(x)}\right),(3.22)$ assume a forma simplificada

$$
\operatorname{TgRatio}(y)=\frac{1}{2}\left(\frac{y_{1}}{y_{D}}-\frac{y_{D}}{y_{1}}, \frac{y_{2}}{y_{D}}-\frac{y_{D}}{y_{2}}, \ldots, \frac{y_{d}}{y_{D}}-\frac{y_{D}}{y_{d}}\right)
$$

Proposição 1. $T_{\text {logEsf }} \circ P_{1}$ e $T_{l o g E s f} \circ P_{2}$ são equivalentes para duas permutaçôes $P_{1}$ e $P_{2}$ se $e$ somente se $P_{1} P_{2}^{-1}$ permuta apenas as duas primeiras componentes das composiçôes (análogo para $T_{\text {tgEsf }}$ com parâmetros lineares).

Prova:

Para $z=\frac{y}{\|y\|}$, temos que $T_{\log E s f}(y)=\left(\log \frac{z_{2}}{z_{1}}, \log \frac{z_{3}}{\sqrt{\sum_{j=1}^{2} z_{j}^{2}}}, \ldots, \log \frac{z_{D}}{\sqrt{\sum_{j=1}^{d} z_{j}^{2}}}\right)$ e, claramente, uma permutação que represente uma transformação linear deve fixar todas as somas sob as raízes 
quadradas.

Corolário 2. $\left\{T_{l o g E s f} \circ P: P\right.$ é uma permutação de $\left.1,2, \ldots, D\right\}$ possui $\frac{D !}{2}$ classes de equivalência distintas (o mesmo para $T_{\text {tgEsf }}$ com parâmetros lineares).

\subsubsection{Observações}

Estatísticos frequentemente procuram procedimentos invariantes sobre mudanças de coordenadas. Sendo assim, alguém poderia questionar o uso das transformações esféricas, já que elas produzem resultados diferentes permutando-se as componentes das composições. Porém, devemos ressaltar que essa mudança de coordenadas não representa apenas um artifício para simplificação de cálculos, mas sim uma mudança sobre todas as hipóteses estatísticas consideradas (de acordo com a definição de transformações equivalentes). É claro que, em cada aplicação, quanto mais estreita a relação entre as hipóteses estatísticas e a natureza dos dados, maior a adequação do modelo. Portanto, se os dados seguem o modelo linear Logaritmo da razão (em um sentido estrito), não será possível obter resultados melhores pela aplicação de outros modelos. Porém, se assumimos que cada hipótese estatística possui a mesma probalidade de ocorrer (assumirmos uma priori uniforme), então provavelmente encontraremos um modelo melhor entre os $\frac{D !}{2}$ gerados por permutações dos modelos esféricos do que o único modelo gerado pelas permutações aplicadas à transformação Logaritmo da razão. Esse parece ser o caso para as composições do Lago Ártico, como mostrado no Capítulo 4, figura 4.3. O melhor modelo Log-esférico apresentou um ajuste mais adequado do que o ajuste pela transformação alr e os outros modelos Log-esféricos tampouco apresentaram pior desempenho.

O modelo Tangente-esférico (com parâmetros lineares) não gerou bons resultados para os mesmos parâmetros fixados nessa análise. Porém, com um pouco mais de insistência, conseguimos produzir (artificialmente) funções $f_{j}$ s de modo a gerar resultados adequados, como mostrado na Figura 4.4.

\subsection{Método dos mínimos quadrados no Simplex}

Por meio da transformação $c l r(3.6)$ e da estrutura euclidiana de $\mathbb{R}^{D}$, Aitchison introduziu no $D$-1-Simplex uma estrutura de $\mathbb{R}$-espaço vetorial ${ }^{6}$ e um produto interno do qual deriva a distância

$$
\Delta(y, w)=\left[\sum_{j=1}^{D}\left(\log \frac{y_{j}}{g(y)}-\log \frac{w_{j}}{g(w)}\right)^{2}\right]^{1 / 2}, g=\text { média geométrica }
$$

\footnotetext{
${ }^{6}$ Mais em geral, se $(V,+,$.$) é um espaço vetorial e \varphi: V \longmapsto B$ é uma função bijetora, então $\varphi$ define um isomorfismo de espaços vetoriais entre $V$ e $B$, com as operações em $B \oplus: B \times B \longmapsto B, \bullet: \mathbb{R} \times B \longmapsto B$ definidas por $b_{1} \oplus b_{2}=\varphi\left(\varphi^{-1}\left(b_{1}\right)+\varphi^{-1}\left(b_{2}\right)\right), \alpha \bullet b=\varphi\left(\alpha \cdot \varphi^{-1}(b)\right)$.
} 
(conhecida como a Distância de Aitchison) que "possui todas as propriedades necessárias para aplicações em análise de dados composicionais ", Aitchison et al. (2000). Da mesma forma,

$$
\left(\sum_{i=1}^{n} \Delta\left(Y_{i}, Z_{i}\right)^{2}\right)^{1 / 2}
$$

define uma distância em $\left(S_{D}\right)^{n}$ que, segundo os critérios de Aitchison, poderia ser considerada como favorita entre as demais distâncias. Logo, observados $\left(x_{1}, y_{1} \bullet\right),\left(x_{2} \bullet, y_{2} \bullet\right), \ldots\left(x_{n} \bullet, y_{n} \bullet\right)$ e dada uma família paramétrica de modelos de regressão $\{h(\mid \beta): \beta \in B\}$, podemos pensar em um método de ajuste do tipo mínimos quadrados, isto é: escolher o modelo que minimize a quantidade

$$
R^{2}(\beta)=\sum_{i=1}^{n} \Delta\left(y_{i \bullet}, h\left(x_{i \bullet} \mid \beta\right)\right)^{2} .
$$

Para variáveis assumindo valores no $D-1$-Simplex, modelos da forma $g=\left(\frac{g_{1}}{\sum g_{j}}, \frac{g_{2}}{\sum g_{j}}, \ldots, \frac{g_{D}}{\sum g_{j}}\right)$, onde $g_{1}\left(x_{\bullet}\right), g_{2}\left(x_{\bullet}\right), \ldots, g_{D}\left(x_{\bullet}\right)$ são funções positivas da covariável $x_{\bullet}$, representam candidatos naturais. De fato, se $y_{1}, y_{2}, \ldots, y_{D}$, são variáveis positivas de mesma espécie modeladas, respectivamente, por $g_{1}\left(x_{\bullet}\right), g_{2}\left(x_{\bullet}\right), \ldots, g_{D}\left(x_{\bullet}\right)$, então a composição das frequências relativas $y=\left(\frac{y_{1}}{\sum y_{j}}, \frac{y_{2}}{\sum y_{j}}, \ldots, \frac{y_{D}}{\sum y_{j}}\right)$ é modelada por por $g$. 


\section{Capítulo 4}

\section{Aplicações}

Os modelos de regressão descritos nos Capítulos anteriores foram implementados utilizando o ambiente de programação $R$ 2.12.2. Para maximização restrita às condições (2.5) foi utilizada a função constrOptim() e, para otimização/minimização irrestrita, a função optim(), exceto para os ajustes de mínimos quadrados linear, para os quais desenvolvemos uma rotina baseada no Teorema do Apêndice A.

Testamos a performance dos modelos propostos para os três conjuntos de dados descritos no Capítulo introdutório, variando os parâmetros (a forma da variável estendida, entre outros). No geral, buscamos os parâmetros que produzissem os melhores ajustes (avaliados visualmente por meio de gráficos) para ilustrar a flexibilidade dos modelos. Para alguns casos, foram necessárias mais do que funções polinomiais (a escolha mais simples e usual).

A seguir apresentamos os parâmtros ótimos (de máxima verossimilhança e de mínimos quadrados) para cada caso, juntamente com o gráfico do ajuste correspondente.

Ao final deste Capítulo encontra-se uma discussão e alguns questionamentos sobre a definição do espaço paramétrico para alguns dos modelos descritos.

\subsection{Lago Ártico}

\subsubsection{Modelo de Dirichlet Linear}

A Figura 4.1 mostra a previsão gerada pelos Modelos de Dirichlet Lineares de primeira (MDL) e segunda (MDQ) ordem ${ }^{1}$ :

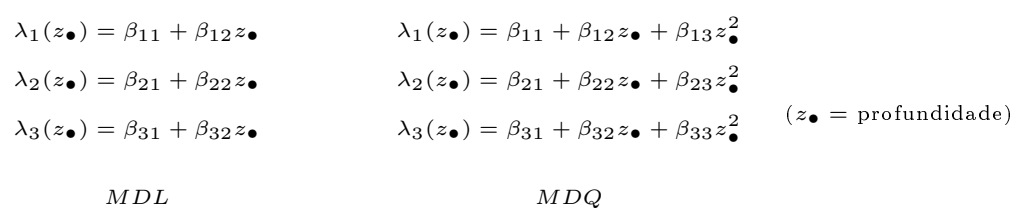

correspondentes aos parâmetros de máxima verossimilhança da Tabela 4.1. Para esses modelos, temos, respectivamente: MDL: $k=2, x_{\bullet}=\left(1, z_{\bullet}\right), \quad$ MDQ: $k=3, x_{\bullet}=\left(1, z_{\bullet}, z_{\bullet}^{2}\right)$.

\footnotetext{
${ }^{1}$ Estes modelos também são abordados em Hijazi e Jernigan (2009).
} 


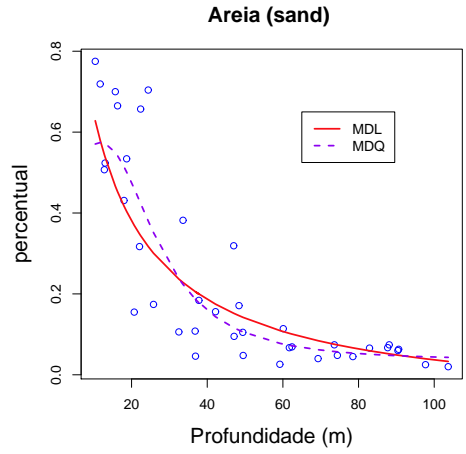

(a)

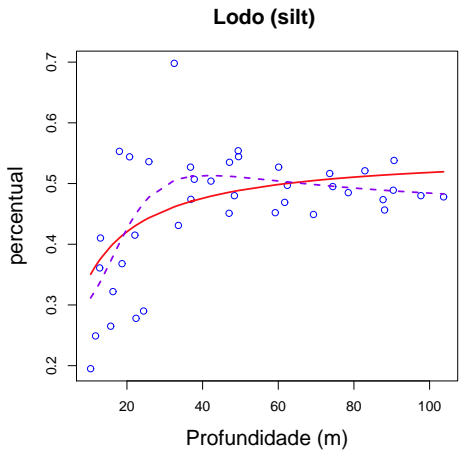

(b)

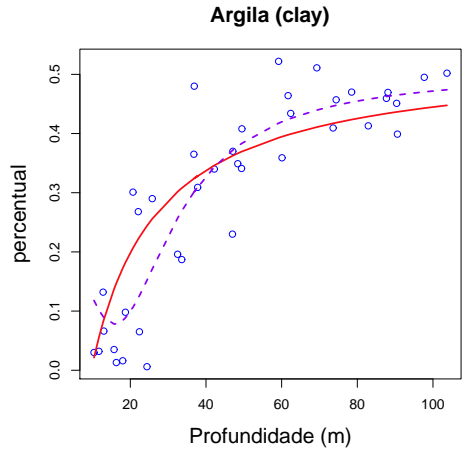

(c)

Figura 4.1: Modelos de Dirichlet lineares

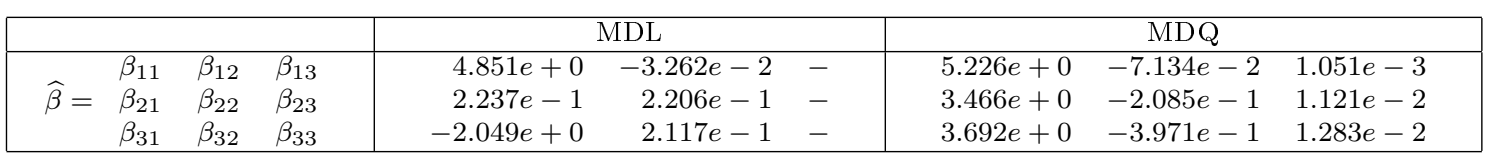

Tabela 4.1: Parâmetros de máxima verossimilhança

\subsubsection{Modelo de Melo, Vasconcellos e Lemonte}

A Figura 4.2 mostra a previsão gerada por três instâncias distintas do Modelo de Dirichlet de Melo, Vasconcellos e Lemonte. A primeira (Mod1) corresponde ao uso da função positiva $1.6+$ $t g^{-1}(u)$. Na segunda (Mod2), aplicamos uma dilatação a esta função para melhorar o ajuste. Na terceira (Mod3), utilizamos a função Exponencial, modificando a forma de $x_{\bullet}$, naturalmente. É importante mencionar que, no caso da função Exponencial, o processo iterativo de otimização não foi completado, pois o valor da função de verossimilhança correspondente excedeu a precisão numérica do $\mathrm{R}\left(10^{307}\right)$.

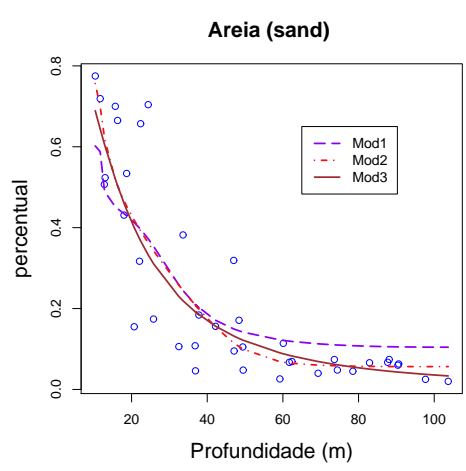

(a)

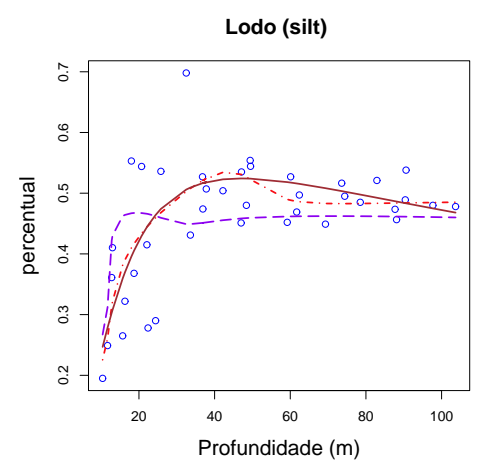

(b)

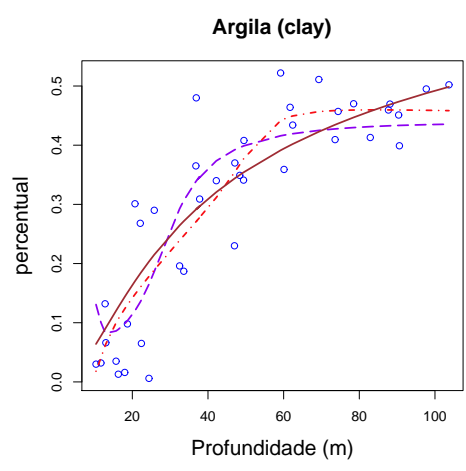

(c)

Figura 4.2: Modelos de Melo, Vasconcellos e Lemonte. 
Legenda:

$z_{\bullet}=$ profundidade

Mod1: $\quad k=3, x_{\bullet}=\left(1, \log \left(0.1 * z_{\bullet}\right), \log \left(0.1 * z_{\bullet}\right)^{0.5}\right.$

$g_{j}(u)=1.6+t^{-1}(u), j=1,2,3$.

$\operatorname{Mod} 2: \quad k=3, x_{\bullet}=\left(1, \log \left(0.1 * z_{\bullet}\right), \log \left(0.1 * z_{\bullet}\right)^{0.5}\right.$

$g_{j}(u)=10 *\left[1.6+t g^{-1}(u)\right], j=1,2,3$.

Mod3: $k=2, x_{\bullet}=\left(1, \log \left(z_{\bullet}\right)\right)$

$g_{j}(u)=\exp (u), j=1,2,3$.

\begin{tabular}{|c|c|c|c|}
\hline & $\widehat{\beta}=$ & $\begin{array}{ll}\beta_{11} & \beta_{12} \\
\beta_{21} & \beta_{22} \\
\beta_{31} & \beta_{32}\end{array}$ & $\begin{array}{l}\beta_{13} \\
\beta_{23} \\
\beta_{33}\end{array}$ \\
\hline \multirow{3}{*}{ Mod1 } & $1.633 e+1$ & $7.612 e+c$ & $-2.311 e+1$ \\
\hline & $2.991 e+0$ & $4.432 e+1$ & $-2.506 e+1$ \\
\hline & $-2.850 e-1$ & $6.786 e+c$ & $-6.668 e+0$ \\
\hline \multirow{3}{*}{ Mod2 } & $2.705 e+0$ & $3.721 e+$ & $-9.541 e+0$ \\
\hline & $5.196 e-1$ & $8.007 e+$ & $-1.050 e+1$ \\
\hline & $-4.829 e+1$ & $-1.871 e+$ & $6.151 e+1$ \\
\hline \multirow{3}{*}{ Mod3 } & $2.482 e-1$ & $3.300 e-1$ & - \\
\hline & $-4.512 e+0$ & $1.924 e+c$ & - \\
\hline & $-7.299 e+0$ & $2.538 e+c$ & - \\
\hline
\end{tabular}

Tabela 4.2: Parâmetros de máxima verossimilhança

\subsubsection{Modelo linear Logaritmo da Razão/ Log-esférico}

A Figura 4.3 compara as performances dos Modelos Logaritmo da Razão (Mod1), do Modelo Log-esférico (Mod3) e dos modelos obtidos pela aplicação do Modelo Log-esférico após permutar as componentes das composições. O ajuste gerado pelo Modelo Mod2 foi ligeiramente superior ao ajuste gerado pela transformação alr (Mod1) e os outros modelos tampoco apresentaram ajustes de qualidade inferior.

Legenda:

$z_{\bullet}=$ profundidade $, \quad k=4, \quad x_{\bullet}=\left(1, z_{\bullet}, z_{\bullet}^{2}, \log \left(z_{\bullet}\right)\right)$

Mod1 : $T=$ alr $\quad \operatorname{Mod} 2: T=T_{\text {logEsf }} \circ P, \quad P\left(y_{1}, y_{2}, y_{3}\right)=\left(y_{1}, y_{3}, y_{2}\right)$

Mod3: $\quad T=T_{\text {logEsf }} \quad \operatorname{Mod} 4: \quad T=T_{\text {logEsf }} \circ P, \quad P\left(y_{1}, y_{2}, y_{3}\right)=\left(y_{3}, y_{2}, y_{1}\right)$ 


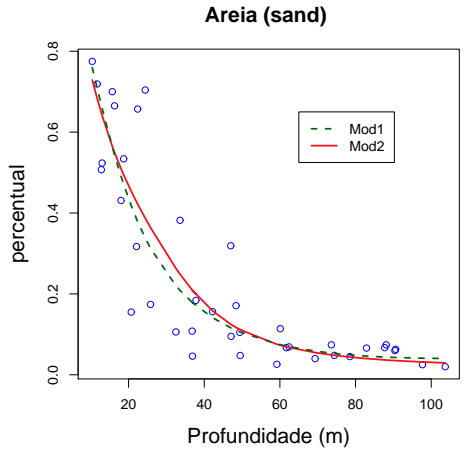

(a)

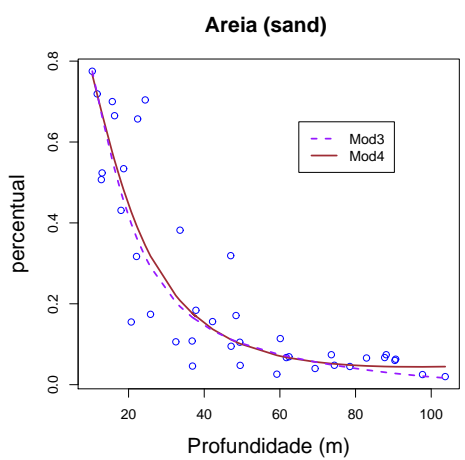

(d)

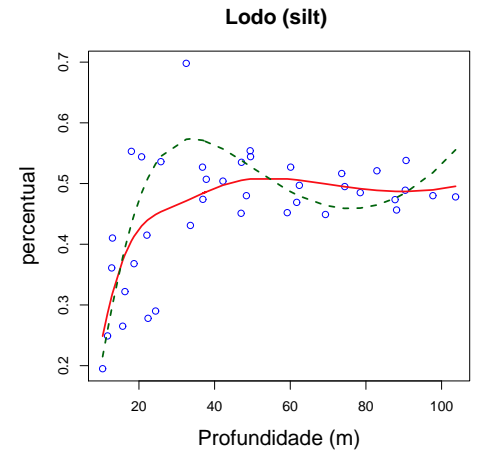

(b)

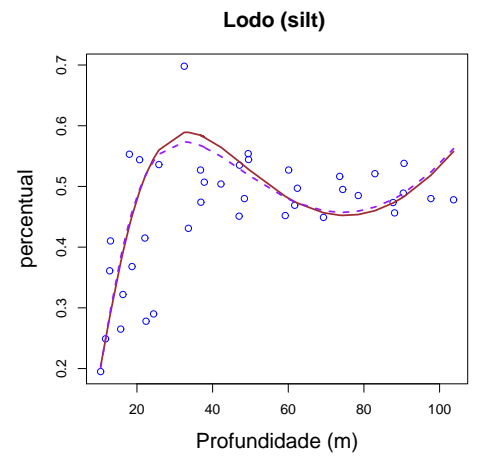

(e)

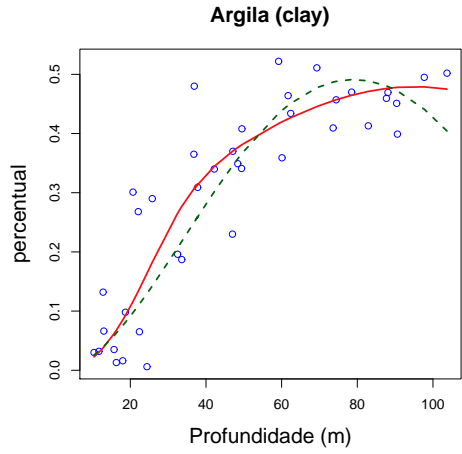

(c)

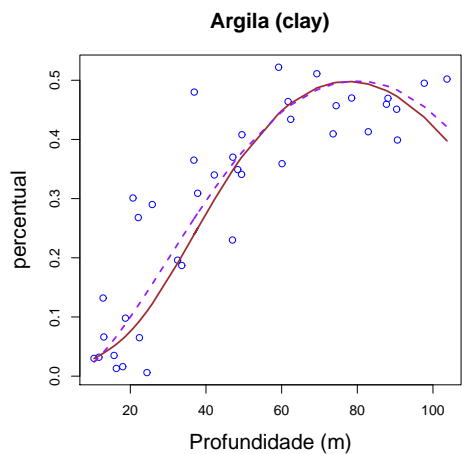

(f)

Figura 4.3: Modelo linear Log-esférico vs Modelo linear Logaritmo da razão

\begin{tabular}{|l|rrrrr|}
\hline & $\widehat{\beta}=$ & $\beta_{11}$ & $\beta_{12}$ & $\beta_{13}$ & $\beta_{14}$ \\
& & $\beta_{21}$ & $\beta_{22}$ & $\beta_{23}$ & $\beta_{24}$ \\
\hline \multirow{2}{*}{ Mod1 } & $8.794 e+0$ & $-6.619 e-2$ & $4.851 e-4$ & $-2.037 e+0$ \\
& $2.060 e+0$ & $-9.804 e-2$ & $5.954 e-4$ & $4.356 e-1$ \\
\hline \multirow{2}{*}{ Mod2 } & $-1.054 e+1$ & $9.998 e-3$ & $-1.439 e-4$ & $2.979 e+0$ \\
& $-6.441 e+0$ & $-9.517 e-2$ & $3.562 e-4$ & $2.697 e+0$ \\
\hline \multirow{2}{*}{ Mod3 } & $-6.956 e+0$ & $-2.885 e-2$ & $6.807 e-5$ & $2.529 e+0$ \\
& $-6.193 e+0$ & $6.684 e-2$ & $-5.190 e-4$ & $9.698 e-1$ \\
\hline \multirow{2}{*}{ Mod4 } & $2.556 e+0$ & $-8.548 e-2$ & $5.394 e-4$ & $1.715 e-1$ \\
& $7.988 e+0$ & $6.635 e-2$ & $-3.874 e-4$ & $-3.114 e+0$ \\
\hline
\end{tabular}

Tabela 4.3: Parâmetros de máxima verossimilhança

\subsubsection{Modelo linear Tangente-esférico}

A Figura 4.4 mostra as estimativas geradas pelo Modelo Tangente-esférico correspondente às estimativas de máxima verossimilhança da Tabela 4.4. Nota-se claramente que a forma de $x_{\bullet}$, assim como no Modelo de Dirichlet de Melo Vasconcellos e Lemonte, foi escolhida artificialmente (modelos polinomiais não geraram bons resultados). Ainda assim, a propriedade de sensibilidade à permutações das composições (ver Corolário 2) foi fundamental para conseguirmos um bom ajuste, pois o Modelo Tangente-esférico (Mod1) não foi suficiente. 


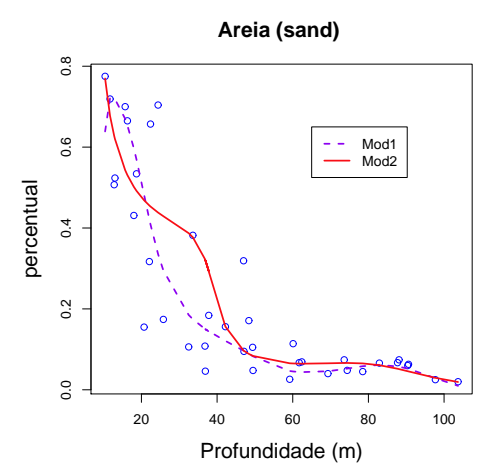

(a)

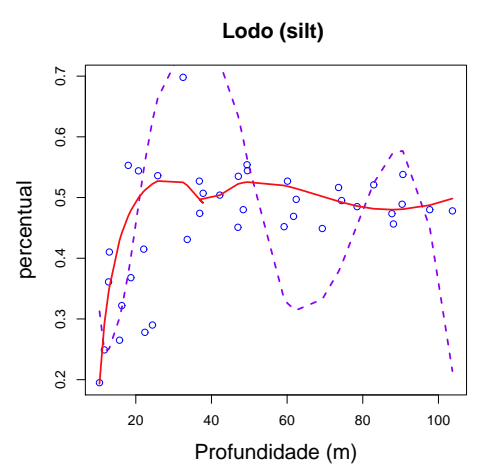

(b)

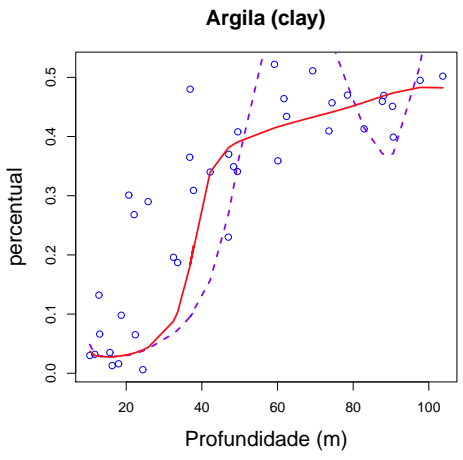

(c)

Figura 4.4: Modelos Tangente-esféricos

Legenda:

$z_{\bullet}=$ profundidade

$k=5, x_{\bullet}=\left(1, z_{\bullet}, z_{\bullet}^{2},\left(-z_{\bullet}+20\right)^{5}, \log \left(z_{\bullet}-10\right)\right)$

Mod1 : $\quad T=T_{T g E s f}$

Mod2: $\quad T=T_{T g E s f} \circ P, \quad P\left(y_{1}, y_{2}, y_{3}\right)=\left(y_{3}, y_{1}, y_{2}\right)$

\begin{tabular}{|c|c|c|c|c|c|}
\hline & & $\begin{array}{l}\beta_{11} \\
\beta_{21}\end{array}$ & $\begin{array}{lll}\beta_{12} & \beta_{13} & \beta \\
\beta_{22} & \beta_{23} & \beta\end{array}$ & $\begin{array}{ll}\beta_{14} & \beta_{15} \\
\beta_{24} & \beta_{25}\end{array}$ & \\
\hline \multirow{2}{*}{ Mod1 } & $-4.978 e+0$ & $3.850 e-1$ & $-3.486 e-3$ & $-3.980 e-9$ & $-6.277 e-1$ \\
\hline & $-2.290 e+1$ & $1.241 e+0$ & $-9.813 e-3$ & $-4.870 e-9$ & $-4.110 e+0$ \\
\hline Mod2 & $\begin{array}{r}2.066 e+1 \\
-1.019 e+0\end{array}$ & $\begin{array}{l}-9.619 e-1 \\
-2.368 e-2\end{array}$ & $\begin{array}{l}8.216 e-3 \\
1.676 e-5\end{array}$ & $\begin{array}{c}6.718 e-9 \\
-8.057 e-11\end{array}$ & $\begin{array}{l}1.291 e+0 \\
6.598 e-1\end{array}$ \\
\hline
\end{tabular}

Tabela 4.4: Parâmetros de máxima verossimilhança

\subsection{Despesas domésticas (bivariado)}

Analisamos a variação dessas composiçoes em função das covariáveis sexo ( $\mathrm{s}=0$, para masculino e $\mathrm{s}=1$, para feminino) e renda $(\mathrm{r})$, isto é: $z_{\bullet}=(s, r)$.

\subsubsection{Modelo de Dirichlet Linear}

A Figura 4.5 mostra o ajuste obtido pelo Modelo de Dirichlet Linear corresponde aos parâmetros da máxima verossimilhança da Tabela 4.5. 


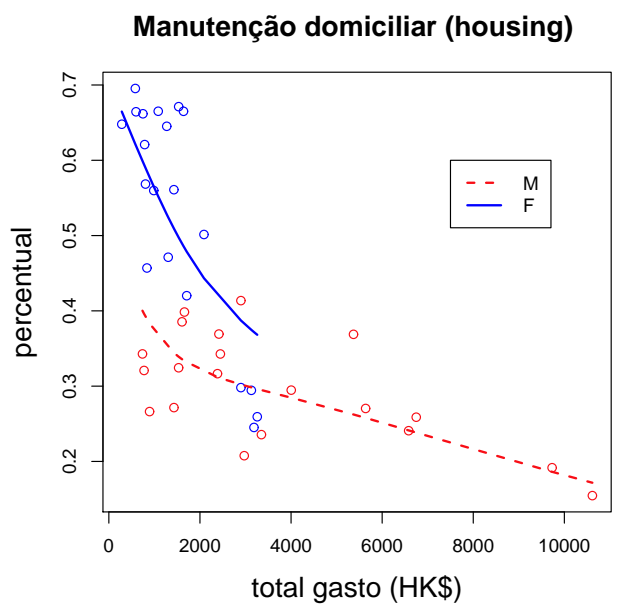

(a)

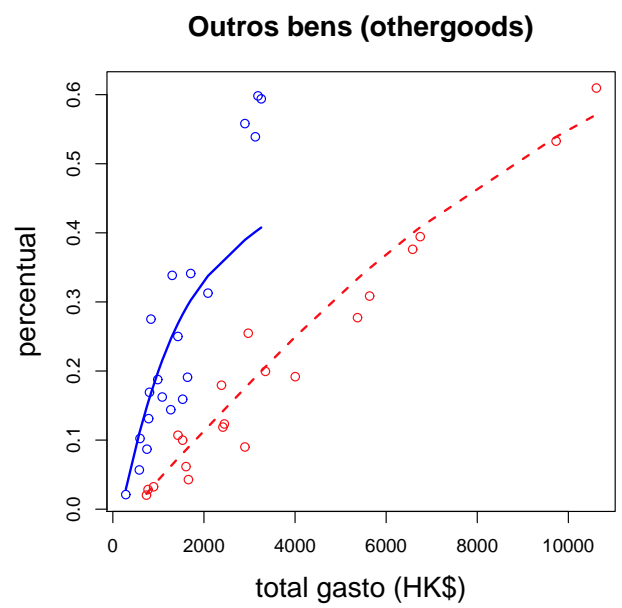

(c)

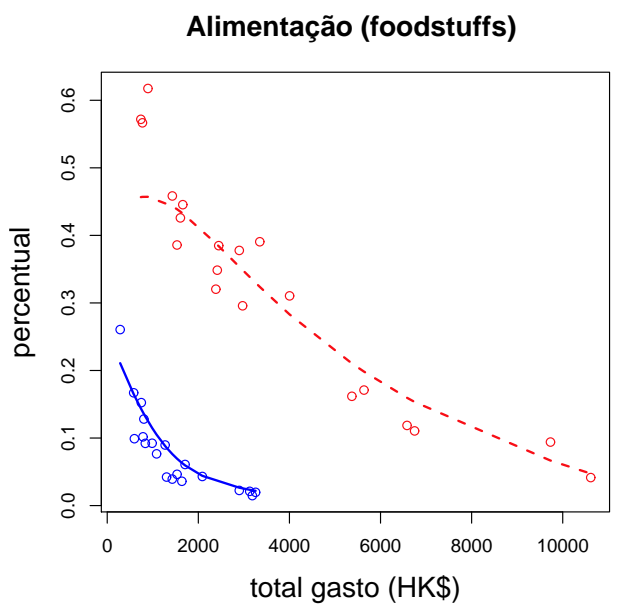

(b)

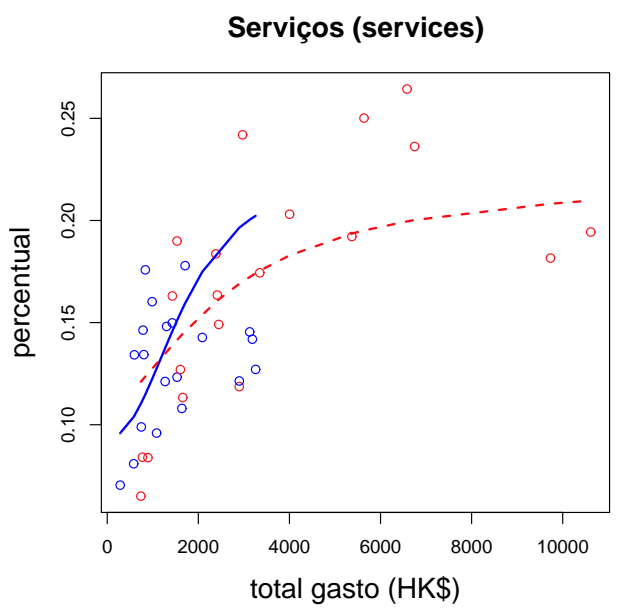

(d)

Figura 4.5: Modelo de Dirichlet linear

Legenda: $\quad k=5, x_{\bullet}=\left(1, s, r, r^{2}, \log (r)\right)$.

\begin{tabular}{|c|c|c|c|c|c|c|c|c|c|}
\hline \multicolumn{5}{|c|}{$\widehat{\beta}=$} & & & & & \\
\hline$\beta_{11}$ & $\beta_{12}$ & $\beta_{13}$ & & $\beta_{15}$ & $1.433 e+2$ & $-6.138 e+0$ & $1.149 e-2$ & $-3.707 e-7$ & $-1.890 e+1$ \\
\hline$\beta_{21}$ & $\beta_{22}$ & $\beta_{23}$ & $\beta_{24}$ & $\beta_{25}$ & $8.032 e+1$ & $-2.561 e+1$ & $4.677 e-3$ & $-3.748 e-7$ & $-8.025 e+0$ \\
\hline$\beta_{31}$ & $\beta_{32}$ & $\beta_{33}$ & $\beta_{34}$ & $\beta_{35}$ & $-2.695 e+1$ & $3.571 e+0$ & $-3.235 e-3$ & $1.599 e-6$ & $4.535 e+0$ \\
\hline$\beta_{41}$ & $\beta_{42}$ & $\beta_{43}$ & $\beta_{44}$ & $\beta_{45}$ & $2.290 e+1$ & $-4.301 e+0$ & $2.832 e-3$ & $2.664 e-7$ & $-2.580 e+0$ \\
\hline
\end{tabular}

Tabela 4.5: Parâmetros de máxima verossimilhança

\subsubsection{Modelo de Melo, Vasconcellos e Lemonte}

A Figura 4.6 mostra o ajuste obtido pelo Modelo de Dirichlet de Melo Vasconcellos e Lemonte correspondente aos parâmetros da máxima verossimilhança da Tabela 4.6. Para esse caso, a função Exponencial não apresentou instabilidade numérica. 


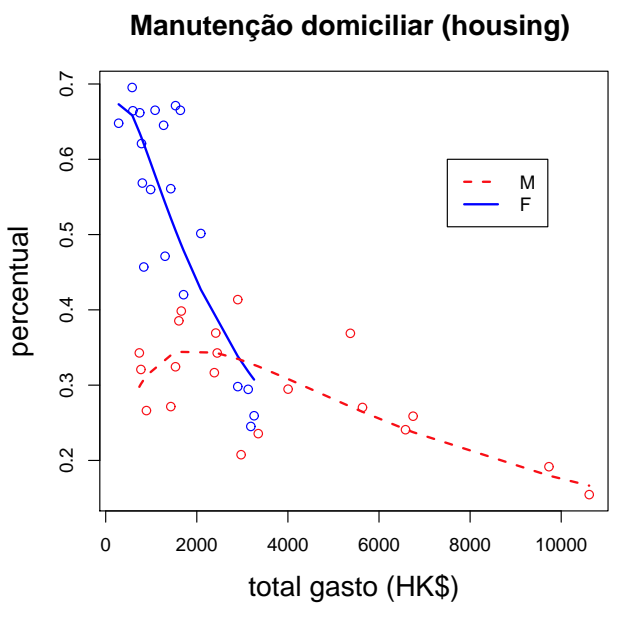

(a)

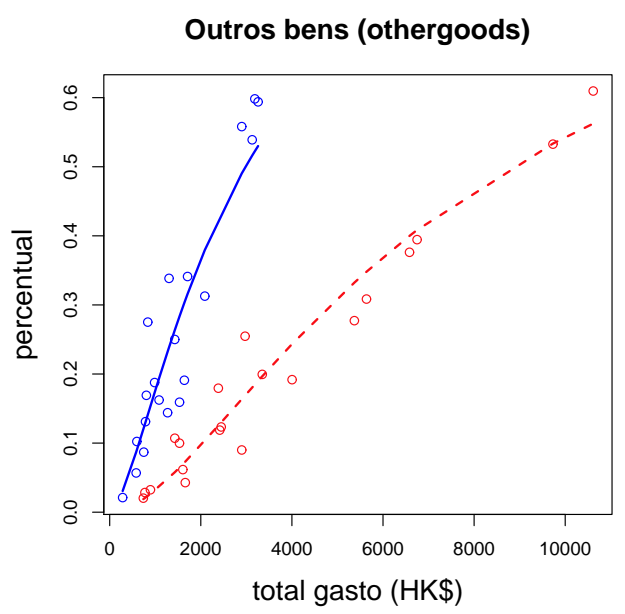

(c)

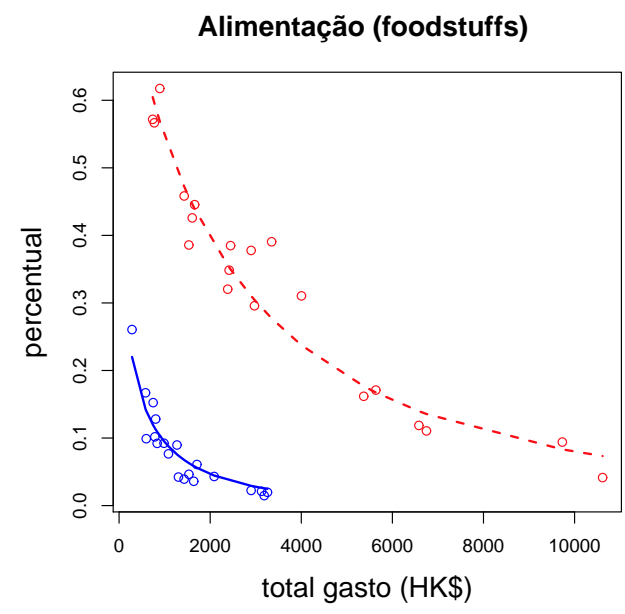

(b)

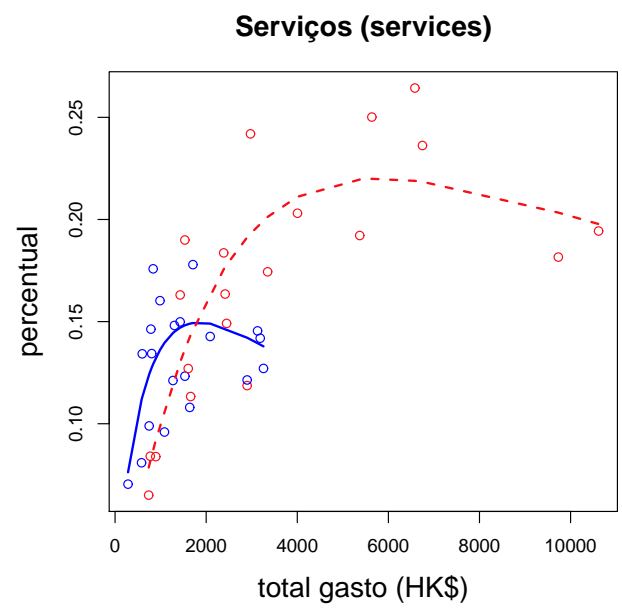

(d)

Figura 4.6: Modelo de Melo, Vasconcellos e Lemonte

Legenda:

$k=3, x_{\bullet}=(1, s, \log (r))$

$g_{j}(u)=\exp (u), j=1,2,3$.

\begin{tabular}{|rrl|rrr|}
\hline \multicolumn{3}{|c|}{$\widehat{\beta}=$} & & & \\
\hline$\beta_{11}$ & $\beta_{12}$ & $\beta_{13}$ & $6.042 e+0$ & $-1.078 e-2$ & $-3.335 e-1$ \\
$\beta_{21}$ & $\beta_{22}$ & $\beta_{23}$ & $1.054 e+1$ & $-2.384 e+0$ & $-9.069 e-1$ \\
$\beta_{31}$ & $\beta_{32}$ & $\beta_{33}$ & $-6.554 e+0$ & $1.077 e+0$ & $1.156 e+0$ \\
$\beta_{41}$ & $\beta_{42}$ & $\beta_{43}$ & $9.840 e-1$ & $-3.180 e-1$ & $2.307 e-1$ \\
\hline
\end{tabular}

Tabela 4.6: Parâmetros de máxima verossimilhança

\subsubsection{Modelo linear TgRatio (3.22)}

O modelo TgRatio não apresentou bons resultados para as composições do Lago Ártico. Na Figura 4.7, apresentamos o bom ajuste gerado por esse modelo para exemplificar a sua utilidade. 


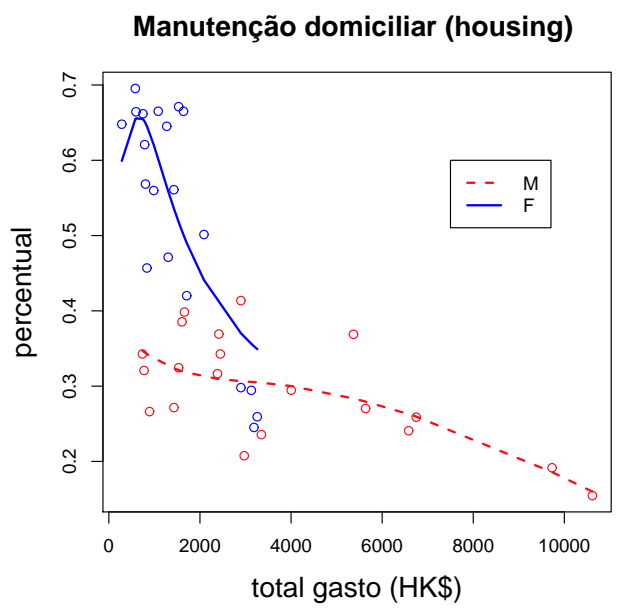

(a)

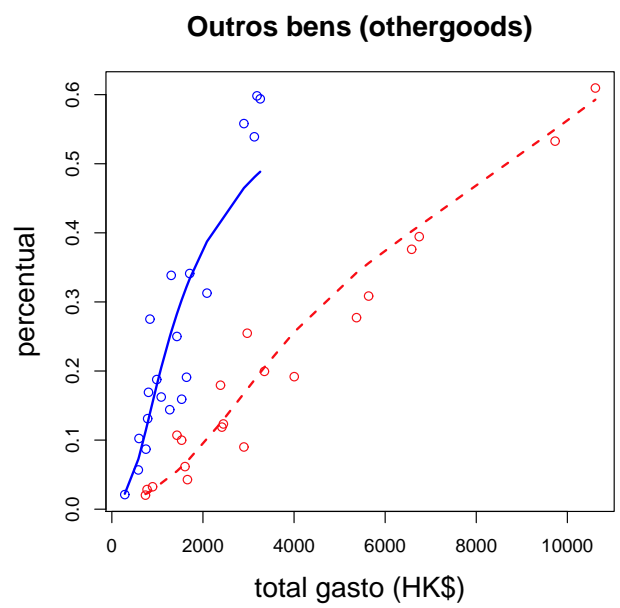

(c)

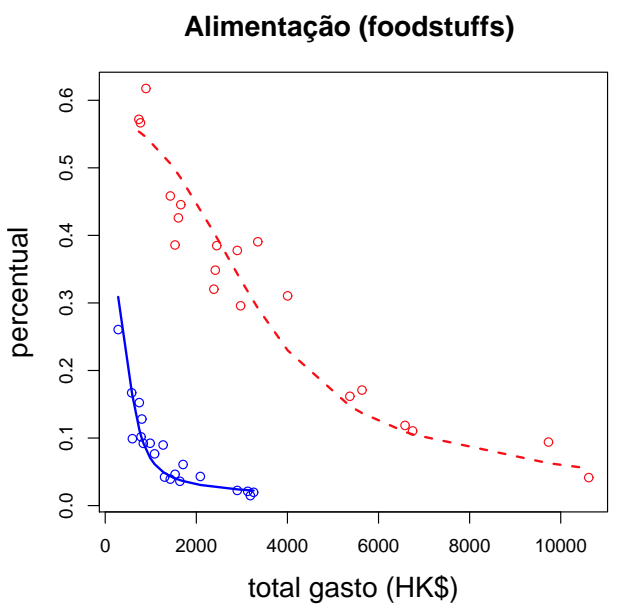

(b)

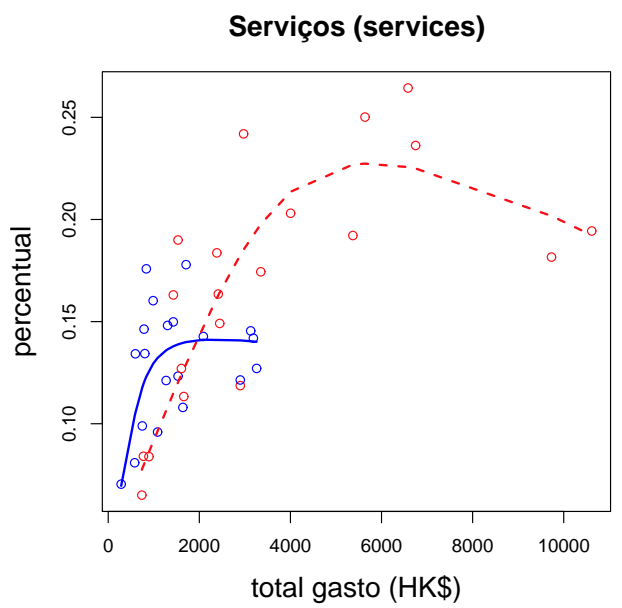

(d)

Figura 4.7: Modelo linear TgRatio

Legenda: $\quad k=5, x_{\bullet}=\left(1, z_{\bullet}, z_{\bullet}^{2}, \log \left(z_{\bullet}\right)\right)$.

\begin{tabular}{|c|c|c|c|c|c|c|c|c|c|}
\hline \multicolumn{5}{|c|}{$\widehat{\beta}=$} & & & & & \\
\hline & & & & $\beta_{15}$ & $2.786 e+1$ & $1.165 e+0$ & $1.027 e-3$ & $-4.407 e-8$ & $-3.685 e+0$ \\
\hline$\beta_{2}$ & $\beta_{22}$ & $\beta_{23}$ & $\beta_{24}$ & $\beta_{25}$ & $3.712 e+1$ & $-7.036 e+0$ & $3.333-4$ & $-1.112 e-8$ & $-4.597 e+0$ \\
\hline$\beta_{3}$ & $\beta_{32}$ & $\beta_{33}$ & $\beta_{34}$ & $\beta_{35}$ & $-2.369 e+1$ & $3.169 e+0$ & $-7.377 e-4$ & $4.319 e-8$ & $3.173 e+0$ \\
\hline
\end{tabular}

Tabela 4.7: Parâmetros de máxima verossimilhança 


\subsection{Casamentos por faixa etária (bivariado)}

Uma covariável dupla $s=(m, w)$ será utilizada para representar o sexo dos individuos: $s=(1,0)$ para homens e $s=(0,1)$ para mulheres. Nos modelos abaixo, utilizamos os dados referentes apenas ao período 1984-1999, para poder comparar as previsões geradas pelos modelos e os dados observados referente aos anos 2000, 2001 e 2002.

\subsubsection{Modelo de Dirichlet Linear}

A Figura 4.8 mostra o ajuste obtido pelo Modelo de Dirichlet Linear.

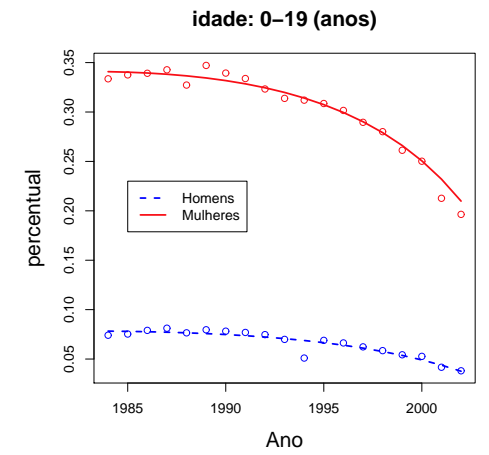

(a)

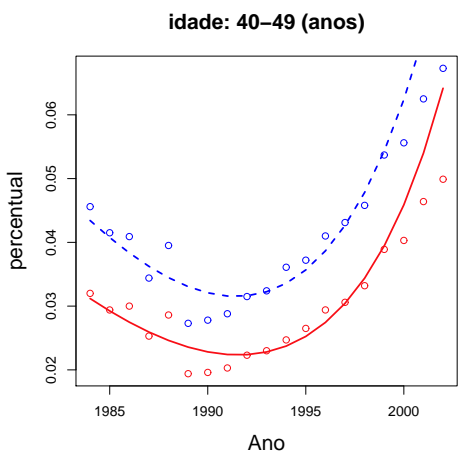

(d)

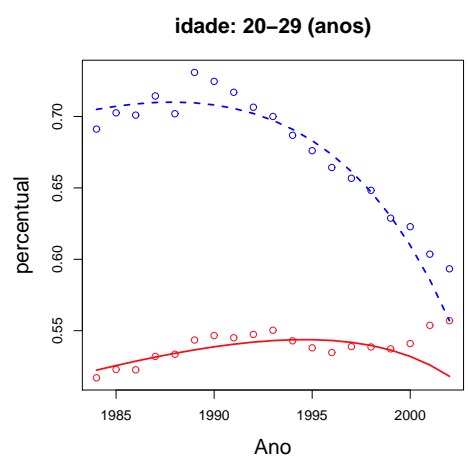

(b)

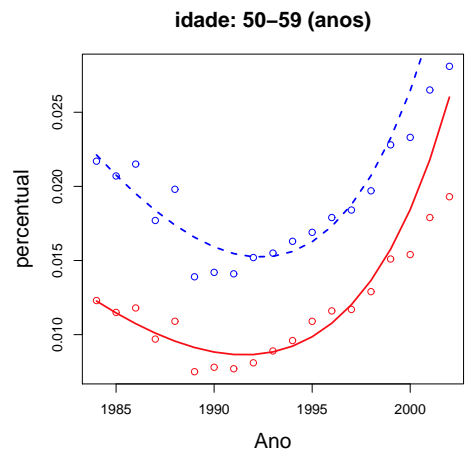

(e)

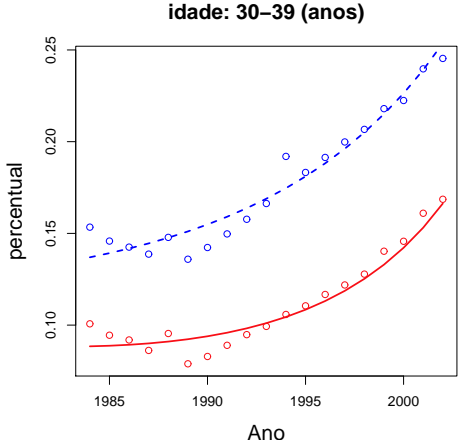

(c)

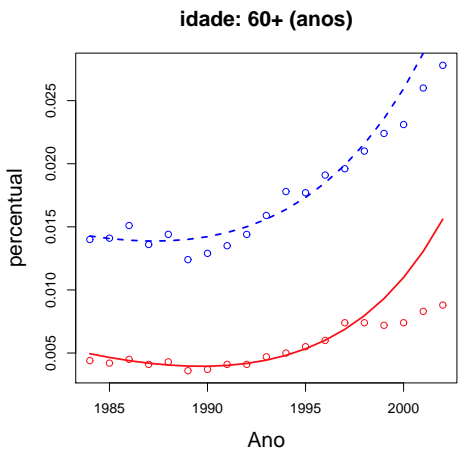

(f)

Figura 4.8: Modelo de Dirichlet Linear

Legenda:

$$
\begin{aligned}
k=7, & x_{\bullet} \\
= & \left(1, w * t, w * t^{2}, w * \cos (t / 100) m * t, m * t^{2}, m * \cos (t / 100)\right) \\
z_{\bullet} & =(m, w, t) s=(m, w)=\text { sexo, } t=\text { ano } .
\end{aligned}
$$




\begin{tabular}{|c|c|c|c|c|c|c|c|c|c|}
\hline & $\widehat{\beta}=$ & $\begin{array}{l}\beta_{11} \\
\beta_{21} \\
\beta_{31} \\
\beta_{41} \\
\beta_{51} \\
\beta_{61}\end{array}$ & $\begin{array}{l}\beta_{12} \\
\beta_{22} \\
\beta_{32} \\
\beta_{42} \\
\beta_{52} \\
\beta_{62}\end{array}$ & $\begin{array}{l}\beta_{13} \\
\beta_{23} \\
\beta_{33} \\
\beta_{43} \\
\beta_{53} \\
\beta_{63}\end{array}$ & $\begin{array}{l}\beta_{14} \\
\beta_{24} \\
\beta_{34} \\
\beta_{44} \\
\beta_{54} \\
\beta_{64}\end{array}$ & $\begin{array}{l}\beta_{15} \\
\beta_{25} \\
\beta_{35} \\
\beta_{45} \\
\beta_{55} \\
\beta_{65}\end{array}$ & $\begin{array}{l}\beta_{16} \\
\beta_{26} \\
\beta_{36} \\
\beta_{46} \\
\beta_{56} \\
\beta_{66}\end{array}$ & $\begin{array}{l}\beta_{17} \\
\beta_{27} \\
\beta_{37} \\
\beta_{47} \\
\beta_{57} \\
\beta_{67}\end{array}$ & \\
\hline$-3.900 e+1$ & $5.059 e+1$ & $-2.545 e$ & -2 & 2.9 & $4 e+3$ & 2.2 & $20 e+0$ & $-1.199 e-3$ & $1.165 e+3$ \\
\hline$-1.030 e+2$ & $5.370 e+1$ & $-2.704 e$ & & 4.9 & $0 e+3$ & 5.2 & $2 e+1$ & $-2.656 e-2$ & $5.098 e+3$ \\
\hline $4.964 e+2$ & $2.441 e+2$ & $-1.195 e$ & & -2.5 & $9 e+4$ & 1.3 & $99 e+2$ & $-6.657 e-2$ & $-1.385 e+4$ \\
\hline $1.023 e+3$ & $3.762 e+2$ & $-1.842 e$ & & -4.0 & $7 e+4$ & 3.7 & $6 e+2$ & $-1.820 e-1$ & $-3.991 e+4$ \\
\hline $4.442 e+2$ & $1.549 e+2$ & $-7.584 e$ & & -1.66 & $3 e+4$ & 1.7 & $38 e+2$ & $-8.510 e-2$ & $-1.859 e+4$ \\
\hline $1.822 e+2$ & $7.204 e+1$ & $-3.527 e$ & & -7.7 ? & $6 e+3$ & 6.7 & $24 e+1$ & $-3.292 e-2$ & $-7.224 e+3$ \\
\hline
\end{tabular}

Tabela 4.8: Parâmetros de máxima verossimilhança

\subsubsection{Modelo de Melo, Vasconcellos e Lemonte}

A Figura 4.9 mostra o ajuste obtido pelo Modelo de Dirichlet de Melo, Vasconcellos e Lemonte. A forma das funções positivas $g_{j}, j=1,2,3,4,5,6$ foi escolhida artificialmente para melhorar a qualidade do ajuste (não conseguimos produzir um ajuste adequado utilizando somente as variantes da função arcotangente, assim como a função Exponencial). O processo de maximização da função de verossimilhança não foi completado, assim como no caso das composições do solo do Lago Ártico.

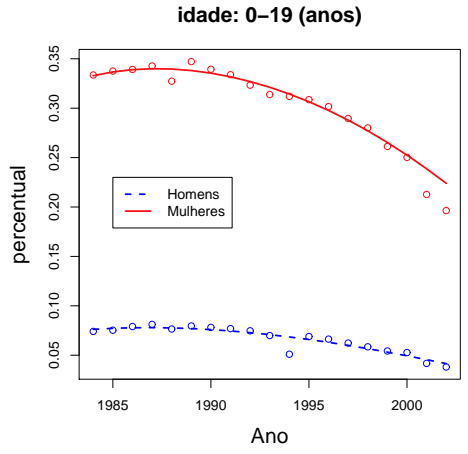

(a)

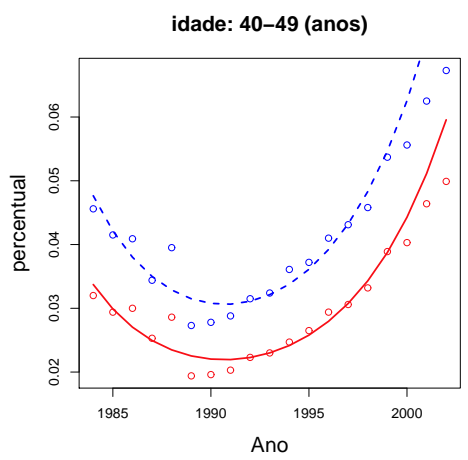

(d)

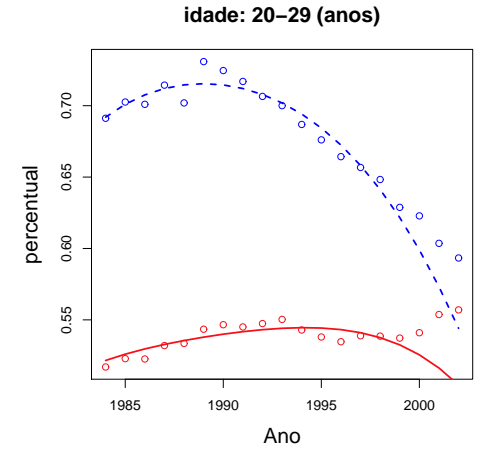

(b)

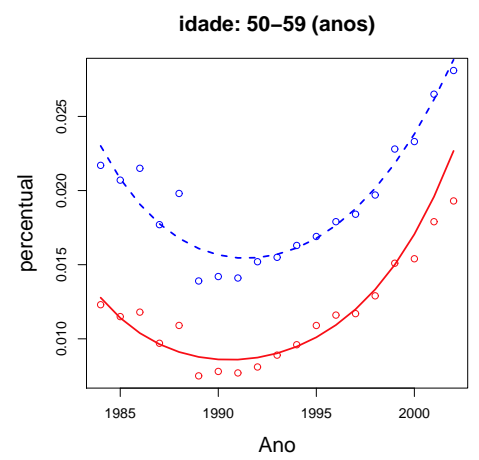

(e)

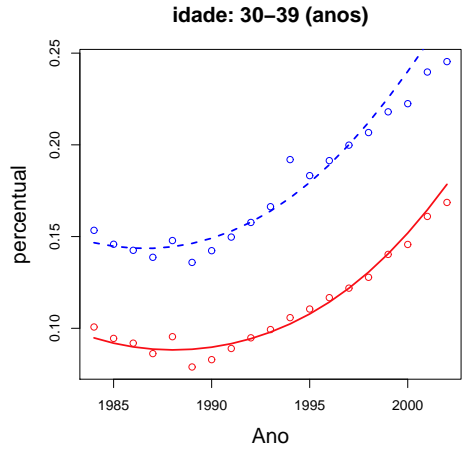

(c)

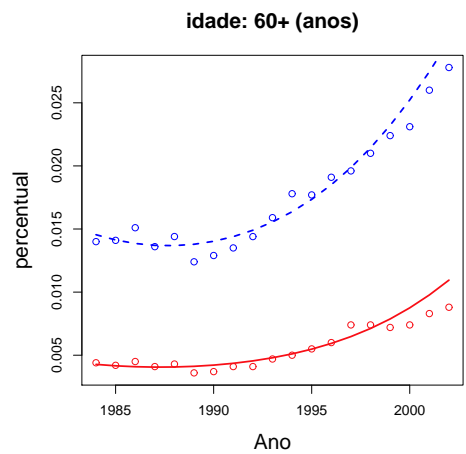

(f)

Figura 4.9: Modelo de Melo, Vasconcellos e Lemonte 
Legenda:

$$
\begin{aligned}
k=7, & x_{\bullet}=\left(1, w * t, w *(t / 10)^{2}, w * \cos (t / 100), m * t, m *(t / 10)^{2}, m * \cos (t / 100)\right) \\
& g_{j}(u)=\left(t g^{-1}(u)+1.6\right) * \exp (u), j=1,2,3,4,5,6 . \\
& z_{\bullet}=(m, w, t), s=(m, w)=\text { sexo, } t=\text { ano. }
\end{aligned}
$$

\begin{tabular}{|c|c|c|c|c|c|c|c|}
\hline & $\widehat{\beta}=$ & $\begin{array}{l}\beta_{11} \\
\beta_{21} \\
\beta_{31} \\
\beta_{41} \\
\beta_{51} \\
\beta_{61}\end{array}$ & $\begin{array}{l}\beta_{12} \\
\beta_{22} \\
\beta_{32} \\
\beta_{42} \\
\beta_{52} \\
\beta_{62}\end{array}$ & $\begin{array}{ll}\beta_{13} & \beta_{14} \\
\beta_{23} & \beta_{24} \\
\beta_{33} & \beta_{34} \\
\beta_{43} & \beta_{44} \\
\beta_{53} & \beta_{54} \\
\beta_{63} & \beta_{64}\end{array}$ & $\begin{array}{ll}\beta_{15} & \beta_{16} \\
\beta_{25} & \beta_{26} \\
\beta_{35} & \beta_{36} \\
\beta_{45} & \beta_{46} \\
\beta_{55} & \beta_{56} \\
\beta_{65} & \beta_{66}\end{array}$ & $\begin{array}{l}\beta_{17} \\
\beta_{27} \\
\beta_{37} \\
\beta_{47} \\
\beta_{57} \\
\beta_{67}\end{array}$ & \\
\hline$-7.347 e+0$ & $-2.784 e+0$ & $1.366 e$ & -1 & $2.887 e+2$ & $-2.487 e+0$ & $1.218 e-1$ & $2.761 e+2$ \\
\hline$-5.121 e+0$ & $-2.193 e+0$ & $1.077 e$ & -1 & $2.227 e+2$ & $-1.968 e+$ & $9.644 e-2$ & $2.181 e+2$ \\
\hline$-5.902 e-1$ & $-1.722 e-1$ & $8.805 e$ & -3 & $4.028 e-1$ & $-5.022 e-$ & $2.605 e-3$ & $5.786 e+0$ \\
\hline $7.088 e+0$ & $2.118 e+0$ & $-1.034 e$ & -1 & $-2.469 e+2$ & $2.799 e+$ & $-1.370 e-1$ & $-3.019 e+2$ \\
\hline $5.340 e+0$ & $1.947 e+0$ & $-9.501 e$ & -2 & $-2.282 e+2$ & $1.722 e+c$ & $-8.426 e-2$ & $-1.837 e+2$ \\
\hline $1.469 e+0$ & $3.917 e-1$ & $-1.887 e$ & -2 & $-6.211 e+1$ & $5.062 e-1$ & $-2.470 e-2$ & $-5.524 e+1$ \\
\hline
\end{tabular}

Tabela 4.9: Parâmetros de máxima verossimilhança

\subsubsection{Modelo linar Log-esférico}

A Figura 4.10 mostra o ajuste obtido pelo Modelo Log-esférico, permutando-se as componentes das composições de forma conveniente.

\begin{tabular}{|c|c|c|c|c|c|c|}
\hline & $\widehat{\beta}=$ & $\begin{array}{l}\beta_{12} \\
\beta_{22} \\
\beta_{32} \\
\beta_{42} \\
\beta_{52}\end{array}$ & $\begin{array}{l}\beta_{13} \\
\beta_{23} \\
\beta_{33} \\
\beta_{43} \\
\beta_{53}\end{array}$ & $\begin{array}{ll}\beta_{14} & \beta_{15} \\
\beta_{24} & \beta_{25} \\
\beta_{34} & \beta_{35} \\
\beta_{44} & \beta_{45} \\
\beta_{54} & \beta_{55}\end{array}$ & $\begin{array}{l}\beta_{16} \\
\beta_{26} \\
\beta_{36} \\
\beta_{46} \\
\beta_{56}\end{array}$ & \\
\hline $2.203 e+3$ & $-1.480 e+0$ & $4.739 e-1$ & & $1.614 e+3$ & $-1.084 e+0$ & $3.484 e-11$ \\
\hline $1.094 e+4$ & $-7.335 e+0$ & $2.330 e-10$ & & $8.200 e+3$ & $-5.503 e+0$ & $1.753 e-10$ \\
\hline $1.947 e+4$ & $-1.304 e+1$ & $4.131 e-1$ & & $1.958 e+4$ & $-1.311 e+1$ & $4.156 e-10$ \\
\hline $1.976 e+4$ & $-1.324 e+1$ & $4.194 e-1$ & & $1.683 e+4$ & $-1.127 e+1$ & $3.569 e-10$ \\
\hline $1.384 e+4$ & $-9.287 e+0$ & $2.953 e-1$ & & $1.020 e+4$ & $-6.845 e+0$ & $2.178 e-10$ \\
\hline
\end{tabular}

Legenda: $\quad T=T_{\log E s f} \circ P, \quad P\left(y_{1}, y_{2}, y_{3}, y_{4}, y_{5}, y_{6}\right)=\left(y_{3}, y_{4}, y_{1}, y_{2}, y_{5}, y_{6}\right)$,

$$
\begin{aligned}
k=6, \quad x_{\bullet} & =\left(w, w * t, w * t^{4}, m, m * t, m * t^{4}\right), \\
z_{\bullet} & =(m, w, t), s=(m, w)=\text { sexo, } t=\text { ano. }
\end{aligned}
$$

Tabela 4.10: Parâmetros de máxima verossimilhança 


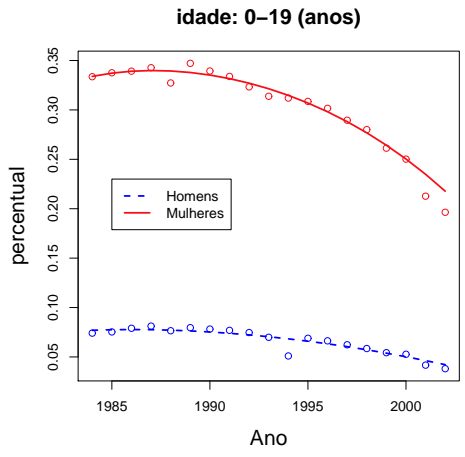

(a)

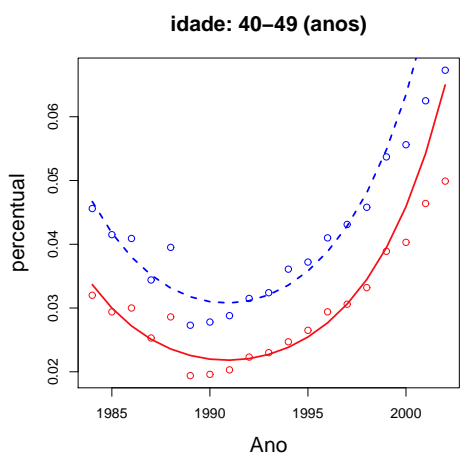

(d)

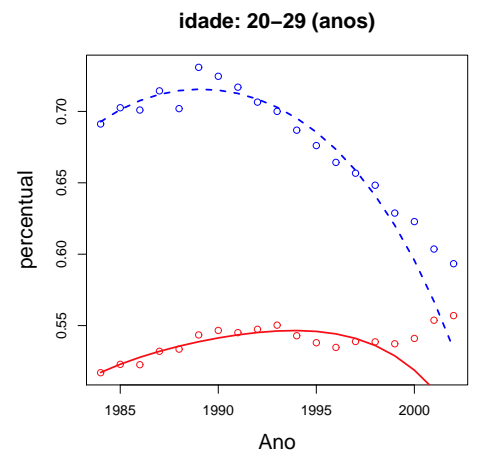

(b)

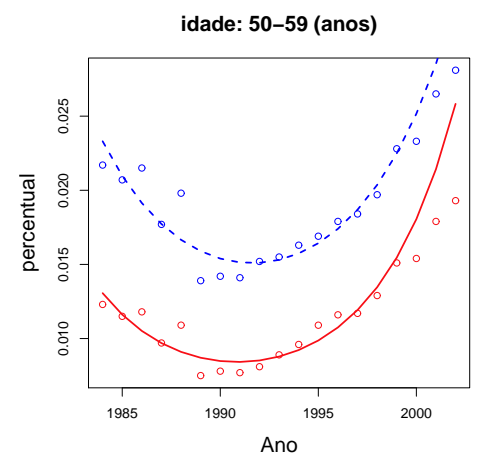

(e)

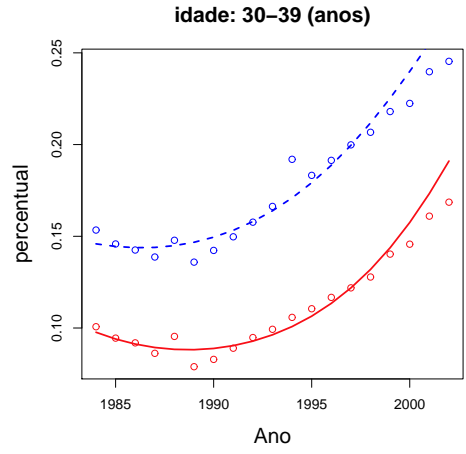

(c)

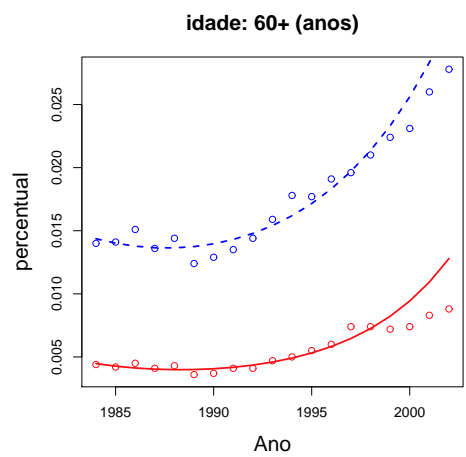

(f)

Figura 4.10: Modelo Log-esférico

\subsection{Considerações sobre o Espaço Paramétrico}

No Capítulo introdutório, foi apresentado um método geral para a obtenção de modelos de regressão, onde a função preditora Prev associa, a cada valor da variável explicativa $x_{\bullet}$, o valor esperado da distribuição com parâmetros $\psi\left(x_{\bullet}, \beta\right)$. Em alguns casos, pode-se querer calcular algumas medidas de dispersão em torno dos valores ajustados, o que pode ser feito calculando (quando possível) os momentos de ordens superiores da distribuição $h\left(. \mid \psi\left(x_{\bullet}, \beta\right)\right)$. Dessa forma, o funcional que associa, a cada vetor/matriz de parâmetros $\beta$, a função $\psi(., \beta)$ é mais completo do que o funcional que associa $\beta$ à função Prev. Porém, se consideramos uma igualdade entre modelos que possuem funções de previsão idênticas, então é preciso reavaliar o método de obtenção da função Prev para alguns dos modelos propostos, pois estes encontram-se descaracterizados.

\subsubsection{Modelo de Dirichlet/ Mínimos quadrados (linear)}

Definição: Dizemos que um subconjunto fechado de $\mathbb{R}^{m}, K$, é um cone se:

- $u+v \in K \forall u, v \in K$.

- $\alpha u \in K, \forall u \in K$ e $\alpha \geq 0$.

O cone $K$ é dito próprio se $K \cap-K=\{0\}$, e sólido, se $K / \partial K \neq \emptyset$. 
Em (2.2), focamos em modelos da forma

$$
g=\left(\frac{\lambda_{1}}{\sum \lambda_{j}}, \frac{\lambda_{2}}{\sum \lambda_{j}}, \ldots, \frac{\lambda_{D}}{\sum \lambda_{j}}\right)
$$

onde $\lambda_{1}(X), \lambda_{2}(X), \ldots, \lambda_{D}(X)$ são dadas por (2.11).

Em termos (2.4), o espaço paramétrico $\Theta$ é constituído por todas as matrizes $\beta$ (ou vetores em $\left.\mathbb{R}^{r * k}\right)$ tais que $\beta \times x_{j} \bullet>0, j=1,2, \ldots, n$. Temos, então, que $\bar{\Theta}=\Theta \cup \partial \Theta$ é um cone próprio e sólido de $\mathbb{R}^{r * k}$. Porém, devido à forma de (4.1), ao multiplicarmos $\beta$ por uma constante positiva, a função preditora $g$ permanece a mesma. Isso, de certa forma, descaracteriza a escolha do estimador de máxima verossimilhança, pois a função de verossimilhança não é invariante sobre dilatações dos seus argumentos e, portanto, existem pontos do espaço paramétrico (cuja verossimilhança é muito baixa) que produzem os mesmos resultados de pontos com verossimilhança alta. O gráfico 4.11 mostra o comportamento da função do logaritmo da verossimilhança (2.8) ao dilatar/contrair os parâmetros do modelo quadrático MDQ da tabela 4.7.

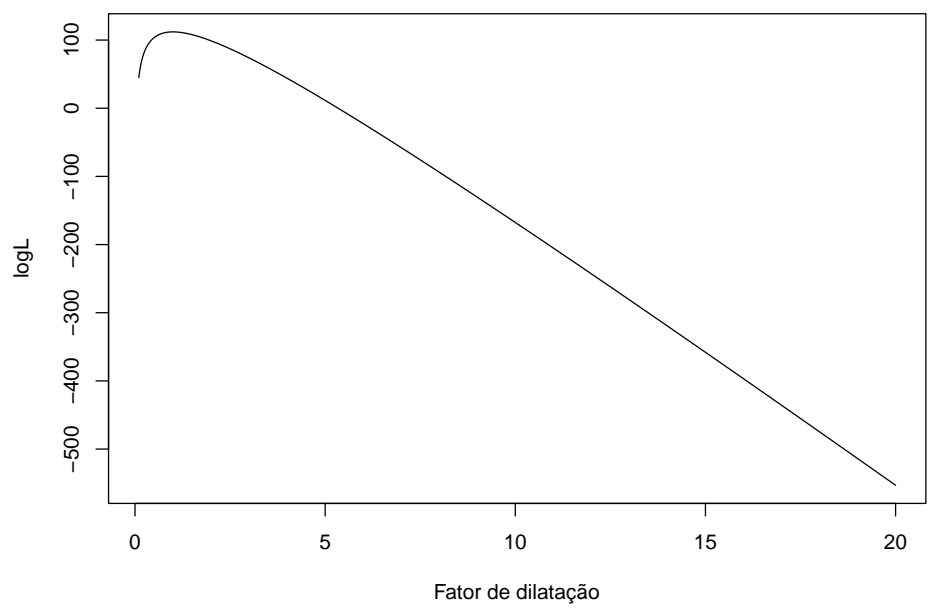

Figura 4.11: Sensibilidade da verossimilhança de $M D Q$ sobre dilatações

Da mesma forma, a soma dos resíduos quadráticos (3.26) é invariante sobre dilatações de $\beta$, para $h$ como em (4.1), de modo que o estimador de mínimos quadrados também fica descaracterizado. Uma forma de eliminar essa ambiguidade é considerar o espaço paramétrico $\Theta^{\prime}=\{\theta \in \Theta:\|\theta\|=1\}$. Utilizando as coordenadas esféricas (3.18), o novo espaço paramétrico fica reduzido a um subconjunto de $[0,2 \pi] \times\left[-\frac{\pi}{2}, \frac{\pi}{2}\right]^{k * r-2}$. Além de "enxugar o excesso de pontos"do espaço paramétrico, trabalhar sobre $\Theta^{\prime}$ (um conjunto limitado) é mais vantajoso, em termos de integração (exata ou numérica), do que trabalhar sobre um conjunto ilimitado. Por outro lado, maximizar a função de verossimilhança nas variáveis $\alpha_{1}, \alpha_{2}, \ldots \alpha_{k * r-1}$ de (3.18) é deveras mais trabalhoso, pois as restrições sobre os novos parâmetros não são mais lineares, como no outro caso. Para o caso do Lago Ártico, conseguimos tanto maximizar a verossimilhança reparametrizada, assim como minimizar a soma dos resíduos quadráticos, redefinindo as funções e os seus gradientes (a valores bem baixos) na região de $[0,2 \pi] \times\left[-\frac{\pi}{2}, \frac{\pi}{2}\right]^{k * r-2}$ não correspondente à $\Theta^{\prime}$. Esse procedimento, porém, produz 
descontinuidades que geram imcompatibilidades na redefinição dos gradientes, de modo que essa prática não é aconselhavel.

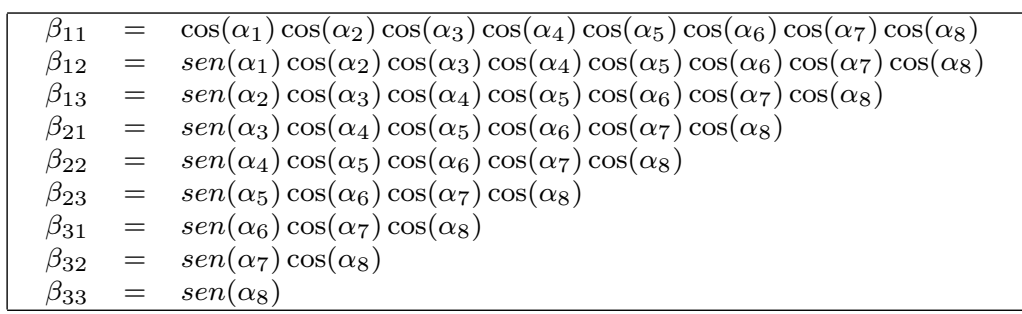

Tabela 4.11: Transformação de $M D Q$ para coordenadas esféricas

\begin{tabular}{|c|c|c|c|c|c|c|c|c|}
\hline & $\alpha_{1}$ & $\alpha_{2}$ & $\alpha_{3}$ & $\alpha_{4}$ & $\alpha_{5}$ & $\alpha_{6}$ & $\alpha_{7}$ & $\alpha_{8}$ \\
\hline MDQ & $2.152 \mathrm{e}-1$ & $-1.291 \mathrm{e}-3$ & $-1.200 \mathrm{e}-1$ & $5.699 \mathrm{e}-2$ & $1.232 \mathrm{e}-2$ & $9.899 \mathrm{e}-1$ & $-1.441 \mathrm{e}-1$ & $8.068 \mathrm{e}-3$ \\
\hline MMQ & $0.000 \mathrm{e}+0$ & $1.320 \mathrm{e}-4$ & $3.454 \mathrm{e}-1$ & $-2.780 \mathrm{e}-2$ & $2.985 \mathrm{e}-3$ & $7.101 \mathrm{e}-1$ & $-8.292 \mathrm{e}-2$ & $2.800 \mathrm{e}-3$ \\
\hline
\end{tabular}

Tabela 4.12: Parâmetros de máxima verossimilhança/ Minimos quadrados

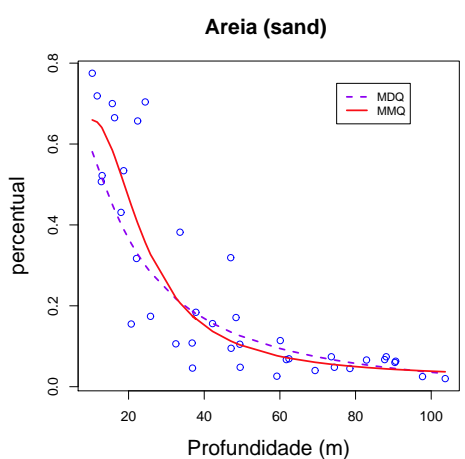

(a)

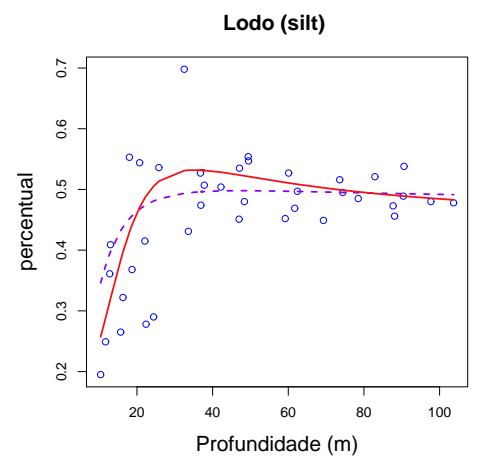

(b)

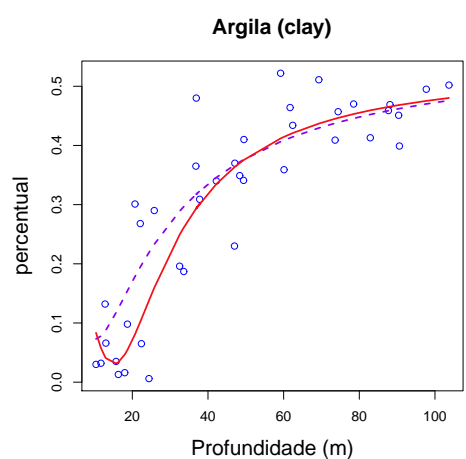

(c)

Figura 4.12: Modelos quadráticos restritos à casca esférica unitária 


\section{Capítulo 5}

\section{Seleção de modelos}

Como vimos na Seção 4.4, o "excesso" de parâmetros em alguns modelos de regressão pode trazer alguns inconvenientes como, por exemplo, a perda da unicidade do estimador de mínimos quadrados. Além disso, como relatado em Melo et al. (2009), a sensibilidade dos estimadores à perturbações pode aumentar consideravelmente com o aumento do número de parâmetros preditores. Por outro lado, modelos simples demais (com uma quantidade muito pequena de parâmetros) podem comprometer o ajuste ao conjunto de dados observados. A obtenção de um equilíbrio entre esses dois extremos, segundo Draper e Smith (1998), pág. 294, constitui o que comumente é designado por "selecionar o melhor modelo de regresssão". Um outro problema de fundamental importância relacionado ao de seleção de modelos é o da análise da dependência da variável resposta em função das variáveis explicativas (covariáveis), visto que, em alguns modelos, essa dependência se traduz na presença/ausência de certos parâmetros. Neste capítulo apresentamos alguns métodos de seleção de modelos baseados em inferência Bayesiana.

\section{$5.1 \quad$ BIC}

O BIC (Bayesian Information Criterion) introduzido por Schwarz (1978) fornece um método relativamente simples para selecionar, dentre um conjunto finito de modelos, o modelo mais simples e representativo para um conjunto de dados observados $Y=\left\{y_{1}, y_{2}, \ldots, y_{n}\right\}$.

Particularidades (segundo Schwarz):

1. O espaço paramétrico $\Theta$ deve ser um subconjunto convexo de $\mathbb{R}^{m}$.

2. Assume-se que a variável y possua distribuição $f(y \mid \theta)=\exp \{T(y) \times \theta-b(\theta)\}$, pertencente à familia exponencial, para algum $\theta \in \Theta$.

3. Os modelos candidatos são da forma $\Theta_{j}=v_{j} \cap \Theta$, onde $v_{j}$ é um subespaço $k_{j}$-dimensional de $\mathbb{R}^{m}(j=1,2, \ldots, s)$. 
Schwarz assume prioris do tipo $\sum_{j=1}^{s} \alpha_{j} \overline{g_{j}}$, onde $g_{1}, g_{2}, \ldots, g_{s}$ são funções densidade de probabilidade definidas respectivamente em $\Theta_{1}, \Theta_{2}, \ldots, \Theta_{s}, \alpha_{1}, \alpha_{2}, \ldots, \alpha_{s}$ são números não negativos tais que $\sum_{j=1}^{s} \alpha_{j}=1$ e $\overline{g_{j}}(\Omega)=g_{j}\left(\Omega \cap \Theta_{j}\right), \forall \Omega \subset \Theta$. A solução ótima corresponde a escolher o modelo mais provável a posteriori, isto é: devemos escolher $j$ de modo a maximizar

$$
S(Y, n, j)=\log \int_{\Theta_{j}}\left(\alpha_{j} \exp \{n(\widetilde{T}(Y) \times \theta-b(\theta))\}\right) d g_{j}^{*}(\theta),
$$

onde $\widetilde{T}(Y)=\frac{1}{n} \sum_{i=1}^{n} T\left(y_{i}\right)$.

O método, que consiste em escolher o modelo que minimiza ${ }^{1}$ a quantidade

$$
B I C=-\max \left\{\log L(\theta \mid Y): \theta \in \Theta_{j}\right\}+\frac{1}{2} k_{j} \log (n),
$$

baseia-se na proposição que afirma que

$$
S(Y, n, j)=n \sup \left\{(\widetilde{T}(Y) \times \theta-b(\theta)): \theta \in \Theta_{j}^{\prime}\right\}-\frac{1}{2} k_{j} \log (n)+R
$$

onde $R$ é uma função limitada em $n$, para $Y$ e $j$ fixados.

A prova é separada em 3 lemas:

Lema 1. A fórmula (5.2) vale se $\widetilde{T}(Y) \times \theta-b(\theta)$ é da forma $A-\lambda\left\|\theta-\theta_{0}\right\|^{2}$, para números positivos $A, \lambda$ e $\theta_{0} \in \Theta$ fixado.

Lema 2. Se as funções densidade de probabilidade de duas variáveis aleatórias limitadas e positivas $U$ e $V$ coincidem onde ambas excedem $\rho$, para algum $\rho, 0<\rho<\sup U$, então

$$
\lim _{n \rightarrow \infty} \log E\left(U^{n}\right)=\lim _{n \rightarrow \infty} \log E\left(V^{n}\right)
$$

Lema 3. Exitem constantes $\lambda_{1}, \lambda_{2}, \rho>0$ tais que

$$
A-\lambda_{1}\left\|\theta-\theta_{j}\right\|^{2}<\widetilde{T}(Y) \times \theta-b(\theta)<A-\lambda_{2}\left\|\theta-\theta_{j}\right\|^{2}
$$

sempre que $\exp \{\widetilde{T}(Y) \times \theta-b(\theta)\}>\rho$, onde $A=\sup \widetilde{T}(Y) \times \theta-b(\theta) e 0<\rho<e^{A}$.

A fórmula (5.2) nos diz que, assintoticamente, o termo limitado pode ser descartado, o que leva à escolha do modelo com o menor $B I C$.

\footnotetext{
${ }^{1}$ Originalmente, o critério de Schwarz propôe escolher o modelo que maximiza $-B I C$.
} 
A proposição de Schwarz é obtida combinando-se os Lemas 1, 2 e 3. O argumento é analogo ao "Teorema do Sanduiche"' para limites: no Lema 1, prova-se a proposição assumindo que a função de verossimilhança possui uma forma específica. O Lema 3 noz diz que, numa vizinhança do ponto de máxima verossimilhança, a função de verossimilhança pode ser prençada entre duas funções para as quais a proposição é válida (pelo Lema 1) e o Lema 2 conclui que, sob essas condições, o resultado vale para a função de verossimilhança.

Embora bastante utilizado em problemas de seleção de modelos, a base matemática apresentada por Schwarz para a sustentação do BIC apresenta algumas inconsistências. Note que, em geral, $\Theta_{j}$ é um subconjunto de medida (de Lebesque) nula em $\Theta$. Segundo os fundamentos da teoria bayesiana ortodoxa, hipótese precisas (hipóteses envolvendo subconjuntos de medida nula do espaço paramétrico) não fazem sentido (Stern (2008), pág 29), pois conjuntos de medida nula possuem probabilidade ZERO. Por conseguinte, nas diversas justificativas criadas para contornar esse tipo de impasse, frequentemente podem ser encontrados argumentos passíveis de erros de interpretação e aparentemente contraditórios (Schwarz admite prioris que atribuem probabilidade não nula aos conjuntos $\Theta_{j} \mathrm{~s}$ ). Além disso, outros argumentos paradoxais são apresentados ao longo do texto:

1. Observe, em primeiro lugar, que a medida $g_{j}^{*}$ é a medida induzida pela função $g_{j}$, isto é:

$$
g_{j}^{*}(\Omega)=\int_{\Omega} g_{j}(\theta) d \theta \quad \forall \Omega \subset \Theta .
$$

No Lema 1, Schwarz assume que $g_{j}^{*}$ é a medida de Lebesgue em $v_{j}$, isto é: $g_{j}^{*}(\Omega)=\int_{\Omega} d \theta$ para todo $\Omega$ mensurável. Isso, obviamente, só é possível se $g_{j} \equiv 1$ (assumindo-se que $g_{j}$ seja, ao menos, contínua). Daí segue que

$$
\int_{\Theta_{j}} d \theta=1
$$

pois $g_{j}$ é uma função densidade de probabilidade. Essa igualdade, porém, não é satisfeita para conjuntos convexos $\Theta$ arbitrários, como suposto pelo autor.

2. Mesmo assumindo a hipótese anterior (o que já nos leva a uma contradição) não há nenhuma outra proposição apresentada pelo autor que estabeleça uma relação deste com o caso geral.

3. Ainda no Lema 1 , supondo que $\widetilde{T}(Y) \times \theta-b(\theta)$ é da forma $A-\lambda\left\|\theta-\theta_{0}\right\|^{2}$, para números positivos $A, \lambda$ e $\theta_{0} \in \Theta_{j}$ fixado, o autor exibe explicitamente o cálculo da integral (5.1)

$$
\int_{\Theta_{j}}\left(\alpha_{j} \exp \left\{n\left(A-\lambda\left\|\theta-\theta_{0}\right\|^{2}\right)\right\}\right) d \theta=\alpha_{j}(\pi / n \lambda)^{k_{j} / 2} e^{n A}
$$

Novamente, a igualdade (5.6) não é satisfeita para conjuntos convexos $\Theta_{j}=\Theta \cap v_{j}$ em geral. 
Para $\Theta_{j}=\mathbb{R}^{k_{j}}$, a fórmula é verdadeira (bem familiar aos introduzidos à distribuição Normal multivariada). Porém, essa hipótese e (5.5) também são contraditórias.

4. É importante ressaltar que, se $\Theta_{i} \subset \Theta_{j}$ propriamente, isto é, $v_{i} \subset v_{j}$ e $\operatorname{dim} v_{i}<\operatorname{dim} v_{j}$, então o uso de medidas distintas em $\Theta_{i}$ e $\Theta_{j}$ é contraditório. Pois, se assumimos a medida de Lebesgue em $\Theta_{j}$, então todo subconjunto de $\Theta_{i}$ tem medida nula em $\Theta_{j}$. Logo, o uso da medida de Lebesgue também em $\Theta_{i}$ é incoerente, pois a probabilidade de $\Theta_{i}$ não pode ser nula e não-nula simultaneamente. Daí conclui-se que o critério de Schwarz faz sentido somente para comparar modelos $\Theta_{i}$ e $\Theta_{j}$ tais que

$$
\Theta_{i} \not \subset \Theta_{j}
$$

Isso, porém, talvez já estivesse implícito nas palavras de Schwarz: "the competing models are...", uma vez que não faz sentido concorrer um modelo com um de seus submodelos.

A seguir, apresentamos uma possível formalização dessas idéias.

\subsubsection{Uma proposta de formalização do $B I C$}

Considere o espaço paramétrico $\Theta^{\prime}=\bigcup_{j=1}^{s} \Theta_{j}$. Se $\mu_{1}, \mu_{2}, \ldots, \mu_{s}$ representam as medidas de Lebesgue em $\Theta_{1}, \Theta_{2}, \ldots, \Theta_{s}$, respectivamente, então $\mu^{\prime}$, dada por $\mu^{\prime}(\Omega)=\sum_{j=1}^{s} \mu_{j}\left(\Omega \cap \Theta_{j}\right)$, é uma medida positiva em $\Theta^{\prime}$. Com essa nova medida, se $g_{1}, g_{2}, \ldots, g_{s}$ são funções densidade de probabilidade (com relação à medida de Lebesgue) definidas, respectivamente, em $\Theta_{1}, \Theta_{2}, \ldots, \Theta_{s}$ e $\alpha_{1}, \alpha_{2}, \ldots, \alpha_{s}$ são números não negativos tais que $\sum_{j=1}^{s} \alpha_{j}=1$, então $\sum_{j=1}^{s} \alpha_{j} \overline{g_{j}}$ representa uma função densidade de probabilidade em $\left(\Theta^{\prime}, \mu^{\prime}\right)\left(\overline{g_{j}}(\Omega)=g_{j}\left(\Omega \cap \Theta_{j}\right)\right)$. Reciprocamente, toda função densidade de probabilidade $h$ (contínua no sentido usual), definida em $\left(\Theta^{\prime}, \mu^{\prime}\right)$, pode ser posta nesse formato, fazendo $\alpha_{j}=\frac{\int_{\Theta_{j}} h d \mu^{\prime}}{\int_{\Theta^{\prime}} h d \mu^{\prime}}$ e $g_{j}=\left.\frac{1}{\alpha_{j}} h\right|_{\Theta_{j}}$, $\left.\operatorname{pois}^{2} \mu^{\prime}\right|_{\Theta_{j}}=\mu_{j}$.

Portanto, podemos assumir, sem perda de generalidade, que a distribuição a posteriori em $\left(\Theta^{\prime}, \mu^{\prime}\right)$ é da forma

$$
\left(\sum_{j=1}^{s} \alpha_{j} g_{j}(\theta)\right) \exp \{n(\widetilde{T}(Y) \times \theta-b(\theta))\},
$$

onde $\widetilde{T}(Y)=\frac{1}{n} \sum_{i=1}^{n} T\left(y_{i}\right)$

\footnotetext{
${ }^{2}$ Por (5.7), se $\Omega \subset \Theta_{j}$, então $\mu_{i}(\Omega)=\mu_{i}\left(\Omega \cap \Theta_{i} \cap \Theta_{j}\right)=0$, pois $v_{i} \cap v_{j}$ possui medida mula em $\Theta_{i}$, para $i \neq j$.
} 
Como mencionado anteriormente, a estratégia de Schwarz consiste em escolher o modelo que maximiza

$$
S(Y, n, j)=\log \int_{\Theta_{j}}\left(\alpha_{j} g_{j}(\theta) \exp \{n(\widetilde{T}(Y) \times \theta-b(\theta))\}\right) d \mu^{\prime}(\theta) .
$$

Suponha que as integrais em (5.9) são definidas sobre subconjuntos da forma

$$
\Theta_{j}^{\prime}=v_{j} \cap\left\{\theta \in \mathbb{R}^{m}:\left\|\theta-\theta_{j}\right\|^{2} \leq r_{j}\right\},
$$

onde $\int_{\Theta^{\prime}} d \mu_{j}=1$ e $\theta_{j}$ é o ponto de máximo da verossimilhança restrita à $v_{j}$. Supondo que, de fato, um (e somente um) ponto do espaço paramétrico corresponde aos dados observados e que o estimador de máxima verossimilhança é consistente (Pawitan (2001), pág. 256), a restrição dos modelos aos conjuntos descritos em (5.10), assim como a escolha da priori, não deve alterar os resultados assintóticos. Portanto, podemos supor que a priori é constante e igual a 1 em cada um dos $\Theta_{j}^{\prime}$ s (obviamente, definimos os $\Theta_{j}^{\prime}$ s de modo a possibilitar uma tal escolha).

As provas dos lemas 2 e 3 dadas por Schwarz são bem acessíveis e não apresentam nenhuma inconsistência, adaptando-se facilmente assumindo (5.10) (recomendamos a leitura de Schwarz (1978) para maiores detalhes). A seguir provamos o Lema 1 sob essas novas hipóteses para obtermos o resultado de Schwarz.

\section{Prova do Lema 1:}

Por indução em $k \geq 2$, defina o sistema de coordenadas:

$$
\left\{\begin{array} { l } 
{ x _ { 1 } ^ { ( 2 ) } = \rho \operatorname { c o s } ( \beta _ { 1 } ) } \\
{ x _ { 2 } ^ { ( 2 ) } = \rho \operatorname { s e n } ( \beta _ { 1 } ) }
\end{array} \quad e \left\{\begin{array}{rl}
x_{1}^{(k+1)} & =x_{1}^{(k)} \cos \left(\beta_{k}\right) \\
& \vdots \\
x_{k}^{(k+1)} & =x_{k}^{(k)} \cos \left(\beta_{k}\right) \\
x_{k+1}^{(k+1)} & =\rho \operatorname{sen}\left(\beta_{k}\right)
\end{array}, \beta_{i} \in \begin{cases}{[0,2 \pi],} & \text { se } i=1 \\
{\left[-\frac{p i}{2}, \frac{\pi}{2}\right],} & \text { se } i>1\end{cases}\right.\right.
$$

O jacobiano é dado por:

$$
\frac{\partial\left(x_{1}^{(k+1)}, x_{2}^{(k+1)}, \ldots, x_{k+1}^{(k+1)}\right)}{\partial\left(\rho, \beta_{1}, \ldots, \beta_{k}\right)}=\left|\begin{array}{ccccc}
\frac{\partial x_{1}^{(k+1)}}{\partial \rho} & \frac{\partial x_{1}^{(k+1)}}{\partial \beta_{1}} & \ldots & \frac{\partial x_{1}^{(k+1)}}{\partial \beta_{k-1}} & \frac{\partial x_{1}^{(k+1)}}{\partial \beta_{k}} \\
\frac{\partial x_{2}^{(k+1)}}{\partial \rho} & \frac{\partial x_{2}^{(k+1)}}{\partial \beta_{1}} & \ldots & \frac{\partial x_{2}^{(k+1)}}{\partial \beta_{k-1}} & \frac{\partial x_{2}^{(k+1)}}{\partial \beta_{k}} \\
\vdots & \vdots & & \vdots & \vdots \\
\frac{\partial x_{k}^{(k+1)}}{\partial \rho} & \frac{\partial x_{k}^{(k+1)}}{\partial \beta_{1}} & \ldots & \frac{\partial x_{k}^{(k+1)}}{\partial \beta_{k-1}} & \frac{\partial x_{k}^{(k+1)}}{\partial \beta_{k}} \\
\frac{\partial x_{k+1}^{(k+1)}}{\partial \rho} & \frac{\partial x_{k+1}^{(k+1)}}{\partial \beta_{1}} & \ldots & \frac{\partial x_{k+1}^{(k+1)}}{\partial \beta_{k-1}} & \frac{\partial x_{k+1}^{(k+1)}}{\partial \beta_{k}}
\end{array}\right|=
$$




$$
\left|\begin{array}{ccccc}
\frac{\partial x_{1}^{(k)}}{\partial \rho} \cos \left(\beta_{k}\right) & \frac{\partial x_{1}^{(k)}}{\partial \beta_{1}} \cos \left(\beta_{k}\right) & \ldots & \frac{\partial x_{1}^{(k)}}{\partial \beta_{k-1}} \cos \left(\beta_{k}\right) & -x_{1}^{(k)} \operatorname{sen}\left(\beta_{k}\right) \\
\frac{\partial x_{2}^{(k)}}{\partial \rho} \cos \left(\beta_{k}\right) & \frac{\partial x_{2}^{(k)}}{\partial \beta_{1}} \cos \left(\beta_{k}\right) & \ldots & \frac{\partial x_{2}^{(k)}}{\partial \beta_{k-1}} \cos \left(\beta_{k}\right) & -x_{2}^{(k)} \operatorname{sen}\left(\beta_{k}\right) \\
\vdots & \vdots & & \vdots & \vdots \\
\frac{\partial x_{k}^{(k)}}{\partial \rho} \cos \left(\beta_{k}\right) & \frac{\partial x_{k}^{(k)}}{\partial \beta_{1}} \cos \left(\beta_{k}\right) & \ldots & \frac{\partial x_{k}^{(k)}}{\partial \beta_{k-1}} \cos \left(\beta_{k}\right) & -x_{k}^{(k)} \operatorname{sen}\left(\beta_{k}\right) \\
\operatorname{sen}\left(\beta_{k}\right) & 0 & \ldots & 0 & \rho \cos \left(\beta_{k}\right)
\end{array}\right|
$$

Por (3.18), vale que $x_{i}^{(k)}=\rho \frac{\partial x_{i}^{(k)}}{\partial \rho}$. Então, desenvolvendo (5.12) por Laplace com relação à última linha, obtemos:

$$
\frac{\partial\left(x_{1}^{(k+1)}, x_{2}^{(k+1)}, \ldots, x_{k+1}^{(k+1)}\right)}{\partial\left(\rho, \beta_{1}, \ldots, \beta_{k}\right)}=\rho \cos \left(\beta_{k}\right)^{k-1} \frac{\partial\left(x_{1}^{(k)}, x_{2}^{(k)}, \ldots, x_{k}^{(k)}\right)}{\partial\left(\rho, \beta_{1}, \ldots, \beta_{k-1}\right)}
$$

Segue que

$$
\frac{\partial\left(x_{1}^{(k)}, x_{2}^{(k)}, \ldots, x_{k}^{(k)}\right)}{\partial\left(\rho, \beta_{1}, \ldots, \beta_{k-1}\right)}=\rho^{k-1} \prod_{i=2}^{k} \cos \left(\beta_{i-1}\right)^{i-2}
$$

Portanto, pelo teorema de mudança de variáveis na integral, obtemos $S(Y, n, j)=$

$$
\log \left[\alpha_{j} e^{n A} \int_{0}^{r_{j}} \int_{0}^{2 \pi} \int_{-\pi / 2}^{\pi / 2} \ldots \int_{-\pi / 2}^{\pi / 2} e^{-n \lambda \rho^{2}} \rho^{k_{j}-1} \prod_{i=2}^{k_{j}} \cos \left(\beta_{i-1}\right)^{i-2} d \beta_{k_{j}-1} \ldots d \beta_{2} d \beta_{1} d \rho\right]
$$

Fazendo $u=n \rho^{2}$ e $M=\int_{0}^{2 \pi} \int_{-\pi / 2}^{\pi / 2} \ldots \int_{-\pi / 2}^{\pi / 2}\left(\prod_{i=2}^{k_{j}} \cos \left(\beta_{i-1}\right)^{i-2}\right) d \beta_{k_{j}-1} \ldots d \beta_{2} d \beta_{1}$, então:

$$
S(Y, n, j)=n A+-\frac{k_{j}}{2} \log (n)+\overbrace{\log \left[\alpha_{j} \frac{M}{2} \int_{0}^{n r_{j}^{2}} e^{-\lambda u} u^{\frac{k_{j}}{2}-1} d u\right]}^{R}
$$

$R$ é limitada em $n$, pois $0<\int_{0}^{n r_{j}^{2}} e^{-\lambda u} u^{\frac{k_{j}}{2}-1} d u \leq \int_{0}^{\infty} e^{-\lambda u} u^{\frac{k_{j}}{2}-1} d u<\infty$. 


\section{$5.2 \quad$ FBST}

Um método elegante e rigoroso para tratar hipóteses precisas, o Full Bayesian Significant Test (FBST), foi apresentado em Pereira e Stern (1999), onde foi introduzido o conceito de valor epistêmico (e-valor) de uma hipótese precisa. O e-valor (ev) é uma medida de crença desenvolvida especialmente (embora não exclusivamente) para avaliar a plausibilidade de uma hipótese precisa sobre o espaço paramétrico.

\subsubsection{Definição formal}

Considere uma família paramétrica de modelos $\{f(y \mid \theta): \theta \in \Theta\}$. Seja $H_{0}: \theta \in \Theta_{H_{0}}$ uma hipótese precisa ${ }^{3}$ sobre $\theta$ e $p_{n}(\theta \mid Y)$ a função densidade de probabilidade a posteriori, dada pela regra de Bayes, após a observação de uma amostra $Y=\left\{y_{1}, y_{2}, \ldots, y_{n}\right\} \subset \Omega$. Inicialmente, o e-valor foi definido da seguinte forma:

$$
e v\left(H_{0}\right)=1-\int_{T\left(H_{0}\right)} p_{n}(\theta \mid Y) d \theta,
$$

onde $T\left(H_{0}\right)=\left\{\theta \in \Theta: p_{n}(\theta \mid y)>\theta_{0}\right\}, \theta_{0}=\arg \max _{\theta \in H_{0}} p_{n}(\theta \mid Y)$. Posteriormente, essa definição foi ligeiramente modificada (Madruga et al. (2003)) para tornar o e-valor invariante sobre mudanças de coordenadas no espaço paramétrico, definindo $\theta_{0}=\arg \max _{\theta \in H_{0}} \frac{p_{n}(\theta \mid Y)}{r(\theta)}$, onde $r$ é uma função densidade de probabilidade de referência escolhida adequadamente (uma para cada teste). A função $s(\theta)=\frac{p_{n}(\theta \mid Y)}{r(\theta)}$ é conhecida como a surpresa a posteriori (posteriori surprise) em relação à função de referência $r$. O conceito de surpresa, discutido em detalhes em Evans (1997), está relacionado à idéia de que "a ocorrência de um valor $x_{0}$ é surpreendente se $f\left(x_{0}\right)=P\left(x_{0}\right)$ é pequeno quando comparado a todos os outros valores possíveis ${ }^{4}$ de $f(x)$ ". Uma maneira de medir a surpresa de um valor $x_{0}$ é comparar o aumento/diminuição da probabillidade a posteriori de $x_{0}$ em relação à probabilidade à priori de $x_{0}$. Isso sugere utilizar uma priori não-informativa como função de referência.

Análogo aos métodos clássicos de inferência, o FBST consiste em designar uma região crítica de rejeição à hipótese: $R C=\left\{Y \subset \Omega: e v\left(H_{0} \mid Y\right)<\bar{e}\right\}$, onde $\bar{e}$ é um limiar fixado a critério do observador. Porém, determinar o quão grande/pequeno deve ser tal limiar está longe de ser uma questão trivial. Uma alternativa a esse impasse, quando disponível, é o uso do $p$-valor (ou nível de significância) (DeGroot e Schervish (2002), pág. 455). Pereira et al. (2008) apresentam uma aproximação do $p$-valor para o FBST utilizando o Teorema da Aproximação Normal para o estimador de máxima verossimilhança (Schervish (1997), página 421), juntamente com a prova da consistência do $e$-valor, sob essas hipóteses.

\footnotetext{
${ }^{3}$ Assume-se que $\Theta_{H_{0}}$ é uma variedade com dimensão menor do que a dimensão de $\Theta$.

${ }^{4}$ Evans se refere a um modelo discreto, nessa ocasião.
} 


\subsection{Teste da Razão de verossimilhança (LR)}

Um dos testes mais conhecidos, o teste da Razão de verossimilhança (likelihood-ratio test Pawitan (2001), pág. 258), consiste em designar uma região crítica baseada na estatística

$$
\Lambda(x)=\frac{\sup \left\{L(x \mid \theta): \theta \in \Theta_{H_{0}}\right\}}{\sup \{L(x \mid \theta): \theta \in \Theta\}}
$$

onde $L$ é a função de verossimilhança, $x$ representa o conjunto de dados observados e $\Theta$ e $\Theta_{H_{0}}$ representam o espaço paramétrico e o subconjunto de $\Theta$ correspondente à hipótese $H_{0}$, respectivamente. Como $\Theta_{H_{0}} \subset \Theta$, a estatística $\Lambda(x)$ assume valores no intervalo [0,1]. Quanto maior esse valor, maior a evidência a favor da hipótese. A aplicabilidade desse teste (em geral) se deve ao Teorema de Wilks Wilks (1938), o qual afirma que a distribuição de $-2 \log \Lambda(x)$ (sob a hipótese $H_{0}$ ) aproxima-se assintoticamente à distribuição $\chi^{2}$ (qui-quadrado) com grau de liberdade igual à diferença entre as dimensões de $\Theta$ e $\Theta_{H_{0}}$. Isso possibilita a obtenção de aproximações do p-valor por meio da substituição da distribuição de $\Lambda(x)$ pela distribuição qui-quadrado.

\subsection{FBST vs LR}

As inconsistências geradas pela necessidade de incluir hipóteses precisas no contexto da teoria bayesiana ortodoxa (Seção 5.1) induzem ao uso do FBST em lugar dos demais testes apresentados acima. Em contrapartida, o BIC e o LR são de fácil implementação, o que não ocorre com o FBST. Isso, obviamente, se deve à indisponibilidade de algoritmos eficientes para o cálculo de integrais numéricas em dimensões elevadas ${ }^{5}$. Para esse fim, algoritmos baseados em métodos de Monte Carlo (Robert e Casella (2004)) parecem ser os mais adequados. A seguir, comparamos as performances do FBST e do LR para um teste de hipótese específico.

Descrição do experimento.

O teste consiste em testar a plausibilidade do modelo de Dirichlet linear (MDL) em relação ao modelo de Dirichlet quadrático (MDQ) da Seção 4.1, isto é:

$$
H_{0}: \beta_{i 3}=0, i=1,2,3 \quad \text { vs } \quad H: \beta_{i j}=1, i, j=1,2,3 .
$$

Para cada valor de $n \in\{30,40,50,60\}$, foram geradas dois grupos $\mathcal{C}_{1}$ e $\mathcal{C}_{2}$ com $m=500$ amostras independentes com $n$ elementos cada(com reposição). As amostras do grupo $\mathcal{C}_{1}$ e $\mathcal{C}_{2}$ correspondem, respectivamente, a conjuntos de dados composicionais obtidos pelas fórmulas (2.2) e (2.6) (mais uma pequena perturbação normal com média zero) utilizando os parâmetros de máxima verossimilhança correpondentes aos modelos (MDL) e (MDQ) da tabela 4.7.

O desempenho de cada teste (FBST e LR) pode ser medido analizando-se as performances dos erros dos Tipos I e II (rejeitar a hipótese $H_{0}$, sendo ela verdadeira e não rejeitar a hipótese $H_{0}$, sendo ela falsa, respectivamente.). Para cada amostra em $\mathcal{C}_{1}$ e em $\mathcal{C}_{2}$, a hipótese $H_{0}$ foi aceita ou rejeitada com base nas aproximações assintóticas para o p-valor descritas nas sessões anteriores ${ }^{6}$,

\footnotetext{
${ }^{5}$ Observe, por exemplo, que o modelo de Dirichlet linear empregado para modelar as porcentagens de casamentos por faixa etária apresenta 42 parâmetros!

${ }^{6}$ Para o cálculo do e-valor, utilizamos uma priori uniforme imprópria em $\mathbb{R}^{C \times D}$ e um algoritmo de integração
} 
para o erro do Tipo I limitado a $\alpha=5 \%$. Como as amostras provenientes de $\mathcal{C}_{1}$ e de $\mathcal{C}_{2}$ foram geradas dentro e fora de $H_{0}$, respectivamente, a porcentagem das amostras de $\mathcal{C}_{1}$ (respectivamente de $\mathcal{C}_{2}$ ) para as quais $H_{0}$ foi rejeitada (respectivamente aceita) fornece uma estimativa para o erro do Tipo I (respectivamente Tipo II).

Uma outra abordagem consiste em analisar o comportamento do erro do Tipo II produzido (em termos da taxa de aceitação das amostras em $\mathcal{C}_{2}$ ) ao selecionar os pontos de corte que determinam as regiões críticas de rejeição de tal forma que, com esses limiares estabelecidos, a hipótese $H_{0}$ seja rejeitada em apenas $5 \%$ das amostras em $\mathcal{C}_{1}$.

Os resultados seguem abaixo.
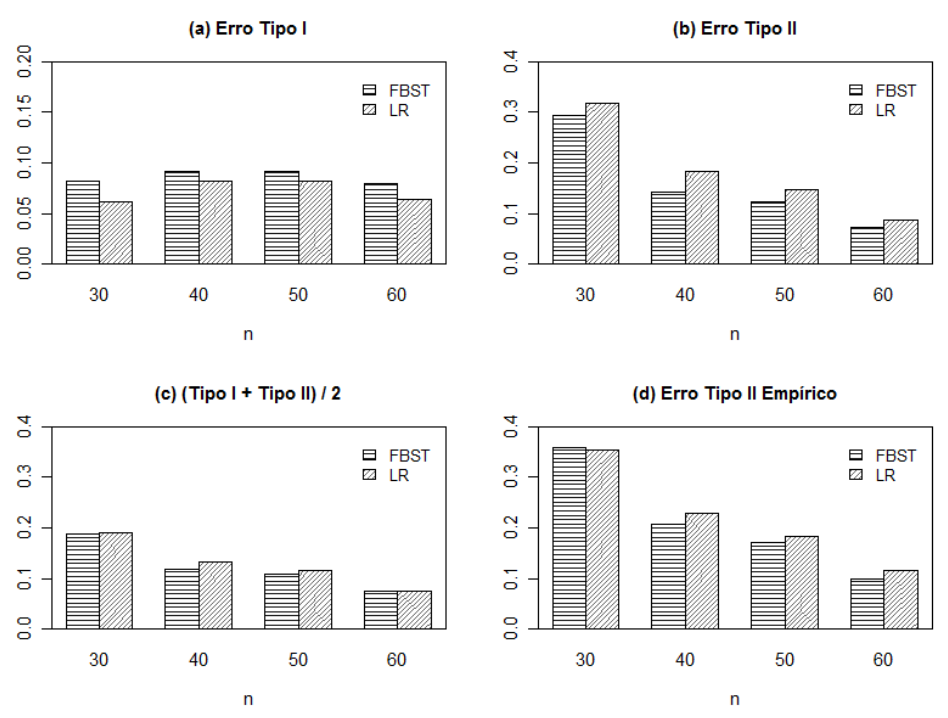

Figura 5.1: (a) Erro Tipo I, (b) Erro Tipo II, (c) Erro médio e (d) Erro Tipo II empírico.

Embora o FBST tenha apresentado uma taxa de erro do Tipo I ligeiramnete maior do que o LR, os erros do Tipo II (assintótico e empírico) e erro médio referentes ao FBST apresentaram valores infeirores aos respectivos referentes ao LR, sugerindo um poder discriminatório maior para o FBST.

\subsection{Aplicações do BIC aos modelos de Dirichlet linear}

\subsubsection{Lago Ártico}

A Figura 5.2 mostra as previsões geradas pelo melhor (menor BIC), pior (maior BIC) e alguns outros modelos correspondentes a valores intermediários de BIC dentre todos os submodelos ${ }^{7}$ do modelo de Dirichlet polinomial quadrático MDQ da Seção 4.1 (os valores correspondentes de BIC para os modelos $M D L$ e $M D Q$ são -95.726 e -82.994).

numérica do tipo Metropolis-Hasting com distribuição normal multivariada como distribuição proposta Martin et al. (2011).

${ }^{7}$ Entende-se por submodelo de um modelo $\bmod$ a todo modelo obtido a partir de $\bmod$ zerando-se, eventualmente, algumas das entradas da sua matriz de modelo. 


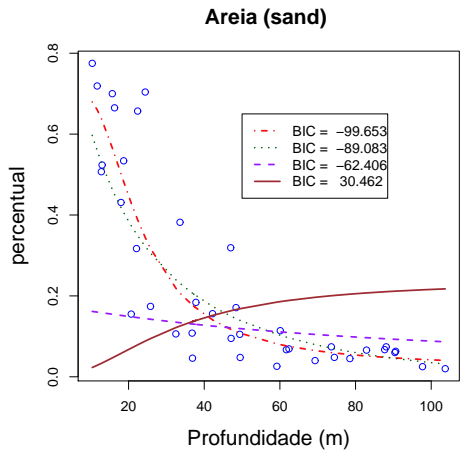

(a)

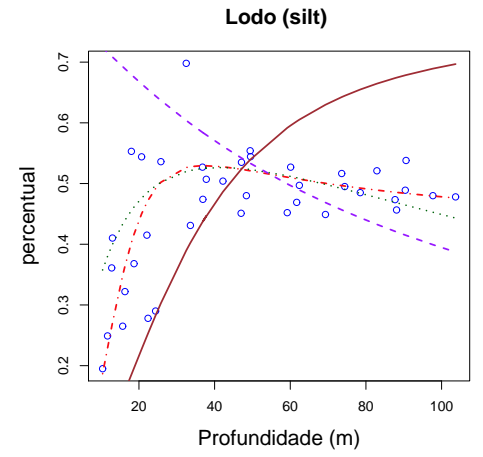

(b)

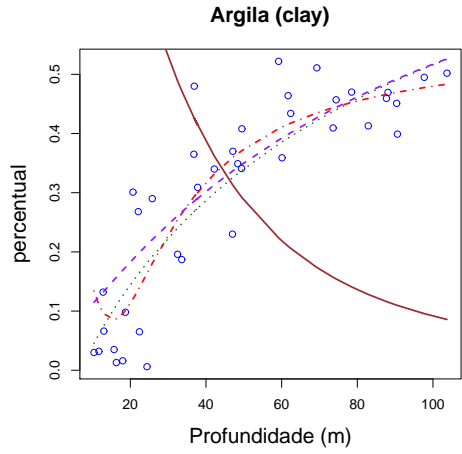

(c)

Figura 5.2: Submodelos de $M D Q$

Observa-se que, conforme o valor do BIC decai, o ajuste é comprometido. Esse fato reitera que os modelos que produzem ajustes ruins possuem pouca chance de ser o modelo correto (são pouco prováveis à posteriori, de acordo com Schwarz).

\begin{tabular}{|c|c|c|c|c|c|c|c|}
\hline \multicolumn{3}{|c|}{ Matriz de modelo } & \multicolumn{3}{|c|}{ Estimativas } & $\mathrm{BIC}$ & Posição \\
\hline $\begin{array}{l}1 \\
0 \\
1\end{array}$ & $\begin{array}{l}0 \\
0 \\
1\end{array}$ & $\begin{array}{l}1 \\
1 \\
1\end{array}$ & $\begin{array}{c}3.545 e+0 \\
- \\
3.033 e+0\end{array}$ & $\begin{array}{c}- \\
- \\
-3.524 e+1\end{array}$ & $\begin{array}{l}4.452 e-4 \\
9.134 e-3 \\
1.239 e+2\end{array}$ & -99.653 & 1 \\
\hline $\begin{array}{l}1 \\
0 \\
1\end{array}$ & $\begin{array}{l}1 \\
1 \\
1\end{array}$ & $\begin{array}{l}0 \\
0 \\
1\end{array}$ & $\begin{array}{c}5.168 e+0 \\
- \\
-5.264 e-1\end{array}$ & $\begin{array}{c}-2.986 e-2 \\
2.800 e-1 \\
5.831 e-2\end{array}$ & $\begin{array}{c}- \\
- \\
2.687 e-3\end{array}$ & -89.083 & 100 \\
\hline $\begin{array}{l}0 \\
0 \\
1\end{array}$ & $\begin{array}{l}1 \\
1 \\
0\end{array}$ & $\begin{array}{l}0 \\
0 \\
1\end{array}$ & $\begin{array}{c}- \\
\overline{-} \\
3.887 e-2\end{array}$ & $\begin{array}{c}3.682 e-2 \\
1.648 e-1 \\
-\end{array}$ & $\begin{array}{c}- \\
\overline{-} \\
2.157 e-3\end{array}$ & -62.406 & 200 \\
\hline $\begin{array}{l}0 \\
0 \\
1\end{array}$ & $\begin{array}{l}0 \\
0 \\
0\end{array}$ & $\begin{array}{l}1 \\
1 \\
0\end{array}$ & $\begin{array}{r}- \\
- \\
7.771 e\end{array}$ & $\begin{array}{rrr} & - & 1.83 \\
& - & 5.86 \\
-1 & - & \end{array}$ & $\begin{array}{l}0 e-4 \\
5 e-4 \\
-\end{array}$ & 30.46160 & 343 \\
\hline
\end{tabular}

Tabela 5.1: Alguns submodelos de MDQ ordenados do menor para o maior BIC

\subsubsection{Despesas domésticas}

Intuitivamente, é de se esperar que homens e mulheres apresentem comportamentos distintos com relação aos gastos com as despesas domésticas. No exemplo 4.2, essa suspeita é incorporada ao modelo de Dirichlet linear por meio da função $f_{2}(r, s)$. Dessa forma, a dependência (ou independência) da distribuição dos gastos com relação ao sexo pode ser matematicamente formulada pela hipótese:

$$
H_{0}: \beta_{i 2}=0, i=1,2,3,4 \quad \text { vs } \quad H: \beta_{i 2} \neq 0, i=1,2,3,4 .
$$

Os BICs relativos aos modelos descritos por $H_{0}$ e por $H$ são iguais a -100.457 e -172.187, respectivamente, donde conclui-se que a hipótese $H_{0}$ deve ser rejeita. Para esse exemplo, em especial, não 
seria necessário tanta sofisticação, pois essa conclusão é imediata a partir de uma análise gráfica dos dados observados. Não há, porém, apelos visuais para determinar (no caso geral) quais das funções $f_{1}(s, r)=1, f_{2}(s, r)=s, f_{3}(s, r)=r, f_{4}(s, r)=r^{2}, f_{2}(s, r)=\log (r)$ são relevantes para esse modelo. Segundo o critério de Schwarz, o melhor modelo $(\mathrm{BIC}=-178.779)$ corresponde ao uso das funções $f_{1}, f_{2}$ e $f_{4}$, apenas.

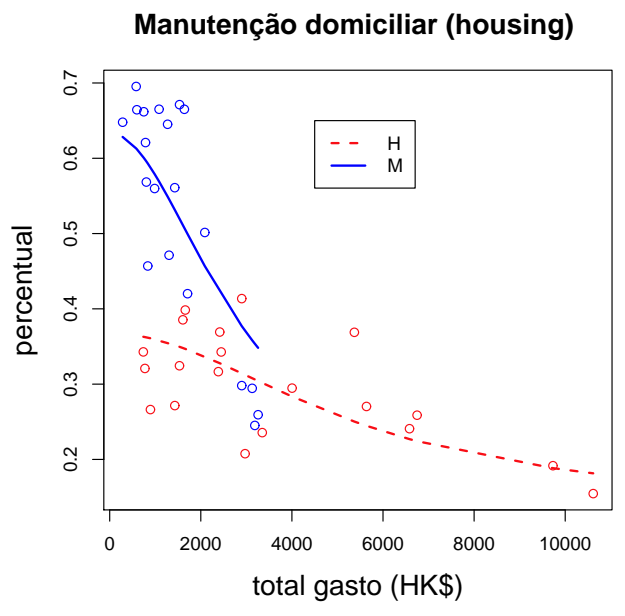

(a)

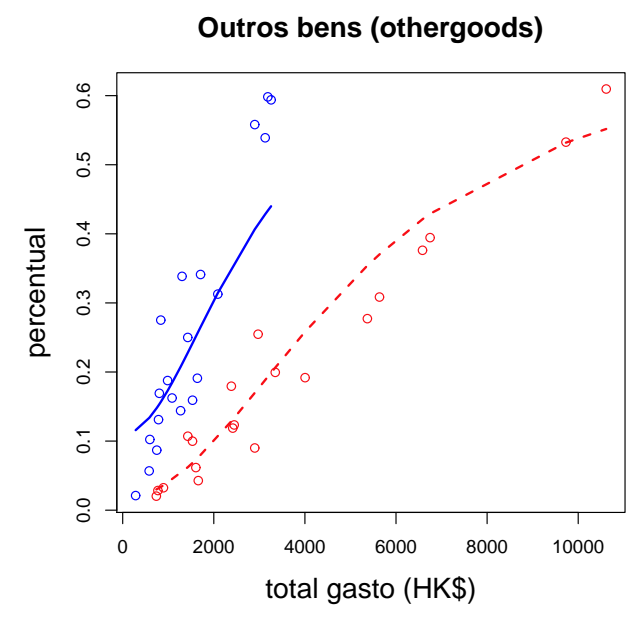

(c)

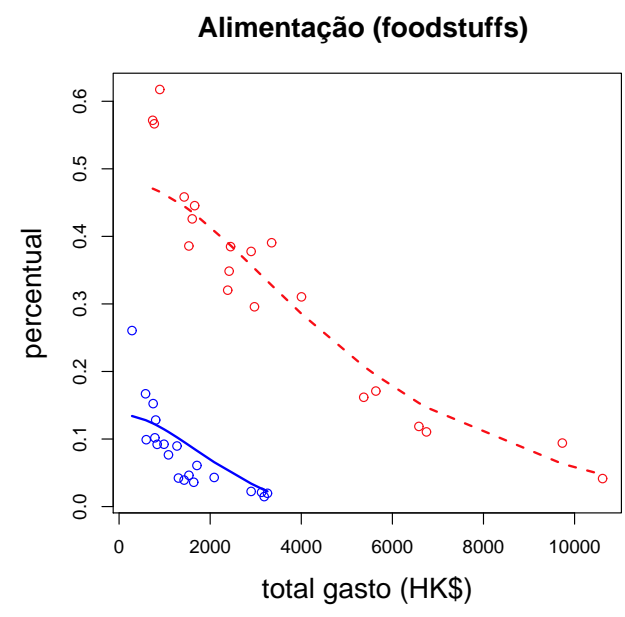

(b)

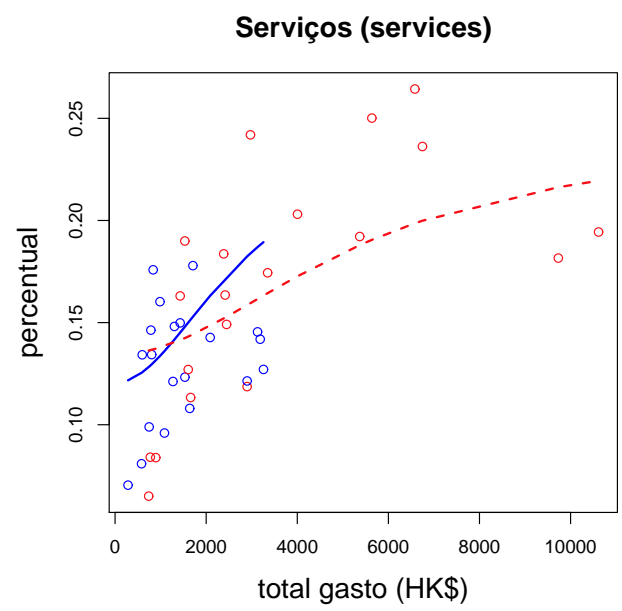

(d)

Figura 5.3: Melhor modelo segundo Schwarz 


\section{Capítulo 6}

\section{Conclusões}

Neste trabalho realizamos um estudo sistemático dos modelos de regressão sobre dados composicionais descritos nos trabalhos de Hijazi e Jernigan (2009), Melo et al. (2009), Aitchinson (1986) e Wang et al. (2007). O primeiro assume que a distribuição condicional da variável resposta, dado o valor da variável explicativa, obedece um Modelo de Dirichlet cujos parâmetros dependem linearmente das variáveis explicativas (covariáveis). O segundo é uma variante do primeiro, no qual funções positivas são aplicadas às combinações lineares das covariáveis para a obtenção dos parâmetros. O terceiro assume que a distribuição condicional dos dados transformados (via transformação Logaritmo da Razão), dado o valor da variável explicativa, segue um modelo Normal multivariado cuja média depende do valor da variável explicativa. O último parametriza o Simplex utilizando coordenadas esféricas generalizadas e ajusta os ângulos diretamente utilizando um modelo de regressão linear.

Também abordamos três métodos de seleção de modelos: BIC, FBST e Teste da razão de verossimilhança.

As principais contribuições obtidas nesse estudo são:

1. A elaboração de um algoritmo mais eficiente para a obtenção de um ponto inicial para o processo iterativo de maximização da função de verossimilhança no Modelo de Dirichlet Linear (Seção 2.3).

2. A completa caracterização das transformações equivalentes (Seção 3.1.1) e a elaboração de novas transformações entre o Simplex e o Espaço eulidiano (Seção 3.3).

3. Um estudo detalhado do critério de Schwarz (BIC).

Os exemplos apresentados no Capítulo 4 mostram que os modelos de Dirichlet Linear, de Melo, Vasconcellos e Lemonte e os modelos baseados em transformações entre o Simplex e o Espaço Euclidiano possuem grande versatilidade. Em alguns casos, porém, a qualidade do ajuste foi devida à escolha artificial da forma de $x_{\bullet}$. Não parece natural, por exemplo, introduzir a componente $m * \cos (t / 100)$ no modelo de Dirichlet linear para o exemplo dos Casamentos por faixa etária. Embora os métodos de seleção de modelo apresentados no Capítulo 5 sirvam para discernir quais das componentes de $x_{\bullet}$ são relevantes, o desafio de escolher adequadamente a forma de $x_{\bullet}$, para cada aplicação, ainda permanece.

Nesse sentido, os modelos baseados em transformações entre o Simplex e o Espaço euclidiano poderiam ser considerados ligeiramente superiores, pois conseguimos bons ajustes utilizando so- 
mente funções polinomiais e logaritmicas. Além disso, tais modelos destacam-se sobre os demais pela fácil implementação e por produzir estimativas sempre dentro do Simplex. Os modelos esféricos, em particular, poderiam ser considerados ligeiramnete superiores ao modelo Logaritmo da Razão de Aitchison por serem sensíveis a permutações das componentes das composições (ver Seção 3.3.1).

O modelo de Melo, Vasconcellos e Lemonte também produz estimativas sempre dentro do Simplex. Porém, além do uso de funções artificiais, a escolha natural para as funções positivas $g_{j}$, a função exponencial, gera grande instabilidade numérica. Uma alternativa é o uso da função arcotangente, como já mencionado na Seção 2.4.

Os modelos de Dirichlet Linear e de Wang podem ser considerados ligeiramente inferiores por não produzirem estimativas sempre dentro do Simplex. O caso do modelo de Dirichlet Linear, entretanto, não é tão crítico como o do modelo de Wang (ver Seção 3.2). Por outro lado, o Algoritmo (2.3.2) garante uma maior estabilidade ao modelo de Dirichlet Linear, destacando-se sobre o modelo de Melo, Vasconcellos e Lemonte.

Sobre os métodos de seleção de modelos, podemos dizer que não há uma relação de preferência evidente.

Embora a comparação das performances do FBST e do LR induza a uma pequena preferência pelo FBST, podemos considerar que o contraste entre as complexidades de implementação anula um possível favoritismo. Consideramos, por outro lado, que o BIC e o FBST não são concorrentes, mas sim métodos complementares. Pois o BIC não parece ser designado para comparar modelos encaixados (a especialidade do FBST).

Esperamos, num futuro próximo, disponibilizar as rotinas desenvolvidas para a construção dos modelos apresentados como pacotes do software livre de Estatística computacional R. 


\section{Apêndice A}

\section{Cálculo dos coeficientes da regressão linear por mínimos quadrados}

Teorema 2. Se a matriz $X$ de (1.1) possui posto máximo, então o residuo quadrático (3.5) possui um único ponto de mínimo $\widehat{\beta}$. Se, além disso, a matriz de modelo (2.3) é completa e $(\widetilde{\beta}, \widetilde{\Sigma})$ é a estimativa de máxima verossimilhança de (3.4) (supondo que exista e seja única), então $\widetilde{\beta}=\widehat{\beta}$.

Prova:

Fazendo $M_{x_{\bullet}}=\left[\begin{array}{cccc}x \bullet & 0 & \ldots & 0 \\ 0 & x_{\bullet} & \ldots & 0 \\ \vdots & \vdots & \ddots & \vdots \\ 0 & 0 & \ldots & x_{\bullet}\end{array}\right]$, temos que

$$
\mu\left(x_{\bullet}, \beta\right)=p s i\left(x_{\bullet}, \beta\right), M_{x_{\bullet}} \times \beta_{v},
$$

$\operatorname{para} \beta_{v}=\left(\beta_{11}, \beta_{12}, \ldots, \beta_{1 k}, \beta_{21}, \beta_{22}, \ldots, \beta_{2 k}, \ldots, \beta_{d 1}, \beta_{d 2}, \ldots, \beta_{d k}\right)$

Logo, (3.5) assume a forma:

$$
\operatorname{Res}^{2}(\beta)=\|\underbrace{\left[\begin{array}{c}
y_{1}^{(t r)} \\
y_{2 \bullet}^{(t r)} \\
\ldots \\
y_{n \bullet}^{(t r)}
\end{array}\right]}_{Y_{v}}-\underbrace{\left[\begin{array}{c}
M_{x_{1} \bullet} \\
M_{x_{2} \bullet} \\
\vdots \\
M_{x_{n}}
\end{array}\right]}_{M} \times \beta_{v}\|^{2}
$$

Reescrevendo (A.2) na forma matricial, obtemos $\operatorname{Res}^{2}(\beta)=\left[Y_{v}-M \times \beta_{v}\right]^{t} \times\left[Y_{v}-M \times \beta_{v}\right]=$

$$
\beta_{v}^{t} \times \underbrace{\left[M^{t} \times M\right]}_{A} \times \beta_{v}-2 \underbrace{\left[Y_{v}^{t} \times M\right]}_{b} \times \beta_{v}+\underbrace{Y_{v}^{t} \times Y_{v}}_{c}
$$


Como $X$ possui posto máximo, então ${ }^{1} A$ é positiva definida, e, portanto, a forma quadrática (A.3) assume um único mínimo (no ponto ${ }^{2} \widehat{\beta_{v}}=A^{-1} \times b^{t}$ ).

Por (3.4), temos que

$$
\log L(\beta, \Sigma)=n * \log \left(\frac{1}{(2 \pi)^{d / 2}|\Sigma|^{1 / 2}}\right)-\frac{1}{2} \sum_{i=1}^{n}\left(T\left(y_{i \bullet}\right)-\mu\left(x_{i \bullet}\right)\right)^{t} \times \Sigma^{-1} \times\left(T\left(y_{i \bullet}\right)-\mu\left(x_{i \bullet}\right)\right)
$$

e, portanto,

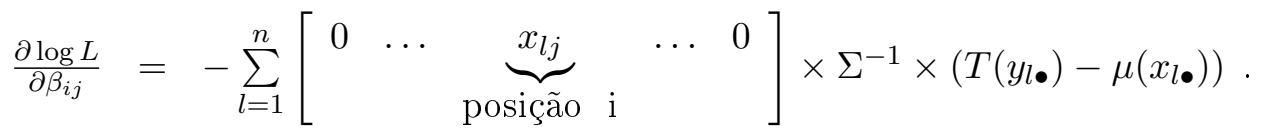

Temos, então, que o sistema $\frac{\partial \log L}{\partial \beta_{i j}}(\beta, \Sigma)=0, i=1,2, \ldots, d$ ( $j$ fixado) equivale a que $\Sigma^{-1} \times\left(\sum_{l=1}^{n} x_{l j}\left(y_{l \bullet}^{(t r)}-\mu\left(x_{l \bullet}\right)\right)\right)$ seja ortogonal ${ }^{3}$ a $\mathbb{R}^{d}$, donde segue que

$$
\sum_{l=1}^{n} x_{l j}\left(y_{l \bullet}^{(t r)}-\mu\left(x_{l \bullet}\right)\right)=0
$$

Como $\log L\left(\beta, I_{d}\right)=n * \log \left(\frac{1}{(2 \pi)^{d / 2}}\right)-\operatorname{Res}^{2}(\beta)$, então $\widehat{\beta} \operatorname{satisfaz}($ A.5) $(\forall j)$ e, como (A.5) não depende de $\Sigma$, segue que

$$
\frac{\partial \log L}{\partial \beta_{i j}}(\widehat{\beta}, \widetilde{\Sigma})=0 \forall i, j
$$

Pela unicidade da estimativa de máxima verossimilhança, obtemos $\widehat{\beta}=\widetilde{\beta}$.

\footnotetext{
${ }^{1}$ Note que $M$ tem posto máximo, pois, rearranjando-se convenientemente as linhas de $M$, obtemos $\left[\begin{array}{cccc}X & & & \\ & X & & \\ & & \ddots & \\ & & & X\end{array}\right]$, que tem posto máximo. Logo, a trasformação $z \longmapsto M \times z$ é injetora e, portanto, $z^{t} \times A \times z=\|M \times z\|^{2}>0$, for $z \neq 0$.

${ }^{2}$ Uma simples manipulação algébrica mostra que $\operatorname{Res}(\widehat{\beta}+\epsilon)=\operatorname{Res}(\widehat{\beta})+\epsilon^{t} \times A \times \epsilon$ (Watkins (2002), pág. 559). ${ }^{3}$ Evidentemente, isso só vale se a matriz de modelo (2.3) for completa.
} 


\section{Apêndice B}

\section{Base de dados}

A seguir apresentamos os conjuntos de dados utilizados nesta dissertação.

1. O primeiro é referente à 39 composições do solo do Lago Ártico (areia, lodo e argila em função da profundidade) apresentados por Coakley e Rust Coakley e Rust (1968) e adaptados por Aitchinson (1986).

2. O segundo é referente ao conjunto de dados encontrado em Aitchinson (1986), constituído pelas despesas domésticas (separadas em Manutenção domiciliar, Alimentação, Outros bens e Serviços.) de 20 homens e 20 mulheres, em função do total gasto.

3. O terceiro corresponde a um conjunto de dados fornecido pelo IBGE (Instituto Brasileiro de Geografia e Estatística) e disponível em http://seriesestatisticas.ibge.gov.br/ relativo à variação das porcentagens de casamentos por faixa etária e sexo no período 1984-2002. 


\section{B.1 Lago Ártico}

\begin{tabular}{|rrrr|rrrr|}
\hline Profundidade $(\mathrm{m})$ & Areia & Lodo & Argila & Profundidade $(\mathrm{m})$ & Areia & Lodo & Argila \\
\hline 10.40 & 0.78 & 0.20 & 0.03 & 47.10 & 0.10 & 0.54 & 0.37 \\
11.70 & 0.72 & 0.25 & 0.03 & 48.40 & 0.17 & 0.48 & 0.35 \\
12.80 & 0.51 & 0.36 & 0.13 & 49.40 & 0.10 & 0.55 & 0.34 \\
13.00 & 0.52 & 0.41 & 0.07 & 49.50 & 0.05 & 0.54 & 0.41 \\
15.70 & 0.70 & 0.26 & 0.04 & 59.20 & 0.03 & 0.45 & 0.52 \\
16.30 & 0.66 & 0.32 & 0.01 & 60.10 & 0.11 & 0.53 & 0.36 \\
18.00 & 0.43 & 0.55 & 0.02 & 61.70 & 0.07 & 0.47 & 0.46 \\
18.70 & 0.53 & 0.37 & 0.10 & 62.40 & 0.07 & 0.50 & 0.43 \\
20.70 & 0.15 & 0.54 & 0.30 & 69.30 & 0.04 & 0.45 & 0.51 \\
22.10 & 0.32 & 0.41 & 0.27 & 73.60 & 0.07 & 0.52 & 0.41 \\
22.40 & 0.66 & 0.28 & 0.06 & 74.40 & 0.05 & 0.49 & 0.46 \\
24.40 & 0.70 & 0.29 & 0.01 & 78.50 & 0.04 & 0.48 & 0.47 \\
25.80 & 0.17 & 0.54 & 0.29 & 82.90 & 0.07 & 0.52 & 0.41 \\
32.50 & 0.11 & 0.70 & 0.20 & 87.70 & 0.07 & 0.47 & 0.46 \\
33.60 & 0.38 & 0.43 & 0.19 & 88.10 & 0.07 & 0.46 & 0.47 \\
36.80 & 0.11 & 0.53 & 0.36 & 90.40 & 0.06 & 0.49 & 0.45 \\
37.80 & 0.18 & 0.51 & 0.31 & 90.60 & 0.06 & 0.54 & 0.40 \\
36.90 & 0.05 & 0.47 & 0.48 & 97.70 & 0.02 & 0.48 & 0.49 \\
42.20 & 0.16 & 0.50 & 0.34 & 103.70 & 0.02 & 0.48 & 0.50 \\
47.00 & 0.32 & 0.45 & 0.23 & & & & \\
\hline
\end{tabular}

Tabela B.1: Composição do solo do Lago Ártico em função da profundidade. 


\section{B.2 Despesas Domésticas}

\begin{tabular}{|c|c|c|c|c|c|c|c|c|c|c|c|}
\hline & $\mathrm{T}$ & M & $\overline{\mathrm{A}}$ & $\mathrm{O}$ & $\mathrm{S}$ & & $\mathrm{T}$ & M & $\bar{A}$ & $\mathrm{O}$ & $\mathrm{S}$ \\
\hline \multirow[t]{20}{*}{ Homens } & 738 & 0.34 & 0.57 & 0.02 & 0.07 & \multirow[t]{20}{*}{ Mulheres } & 284 & 0.65 & 0.26 & 0.02 & 0.07 \\
\hline & 773 & 0.32 & 0.57 & 0.03 & 0.08 & & 581 & 0.70 & 0.17 & 0.06 & 0.08 \\
\hline & 894 & 0.27 & 0.62 & 0.03 & 0.08 & & 596 & 0.66 & 0.10 & 0.10 & 0.13 \\
\hline & 1429 & 0.27 & 0.46 & 0.11 & 0.16 & & 748 & 0.66 & 0.15 & 0.09 & 0.10 \\
\hline & 1532 & 0.32 & 0.39 & 0.10 & 0.19 & & 786 & 0.62 & 0.10 & 0.13 & 0.15 \\
\hline & 1606 & 0.39 & 0.43 & 0.06 & 0.13 & & 804 & 0.57 & 0.13 & 0.17 & 0.13 \\
\hline & 1659 & 0.40 & 0.45 & 0.04 & 0.11 & & 836 & 0.46 & 0.09 & 0.28 & 0.18 \\
\hline & 2385 & 0.32 & 0.32 & 0.18 & 0.18 & & 986 & 0.56 & 0.09 & 0.19 & 0.16 \\
\hline & 2416 & 0.37 & 0.35 & 0.12 & 0.16 & & 1084 & 0.67 & 0.08 & 0.16 & 0.10 \\
\hline & 2448 & 0.34 & 0.38 & 0.12 & 0.15 & & 1271 & 0.65 & 0.09 & 0.14 & 0.12 \\
\hline & 2899 & 0.41 & 0.38 & 0.09 & 0.12 & & 1303 & 0.47 & 0.04 & 0.34 & 0.15 \\
\hline & 2972 & 0.21 & 0.30 & 0.25 & 0.24 & & 1428 & 0.56 & 0.04 & 0.25 & 0.15 \\
\hline & 3349 & 0.24 & 0.39 & 0.20 & 0.17 & & 1533 & 0.67 & 0.05 & 0.16 & 0.12 \\
\hline & 4004 & 0.29 & 0.31 & 0.19 & 0.20 & & 1639 & 0.67 & 0.04 & 0.19 & 0.11 \\
\hline & 5371 & 0.37 & 0.16 & 0.28 & 0.19 & & 1709 & 0.42 & 0.06 & 0.34 & 0.18 \\
\hline & 5637 & 0.27 & 0.17 & 0.31 & 0.25 & & 2088 & 0.50 & 0.04 & 0.31 & 0.14 \\
\hline & 6582 & 0.24 & 0.12 & 0.38 & 0.26 & & 2899 & 0.30 & 0.02 & 0.56 & 0.12 \\
\hline & 6748 & 0.26 & 0.11 & 0.39 & 0.24 & & 3128 & 0.29 & 0.02 & 0.54 & 0.15 \\
\hline & 9731 & 0.19 & 0.09 & 0.53 & 0.18 & & 3186 & 0.25 & 0.01 & 0.60 & 0.14 \\
\hline & 10615 & 0.15 & 0.04 & 0.61 & 0.19 & & 3258 & 0.26 & 0.02 & 0.59 & 0.13 \\
\hline
\end{tabular}

Tabela B.2: Despesas domésticas: $T=$ Total gasto (em HK\$); $A=$ Alimentação; $O=$ Outros Bens; $S=$ Serviços. 


\section{B.3 Casamentos por faixa-etária}

\begin{tabular}{|c|c|c|c|c|c|c|c|}
\hline & Ano & 0 a 19 & 20 a 29 & 30 а 39 & 40 а 49 & 50 а 59 & $60+$. \\
\hline \multirow[t]{19}{*}{ Homens } & 1984 & 7.41 & 69.12 & 15.34 & 4.56 & 2.17 & 1.40 \\
\hline & 1985 & 7.53 & 70.25 & 14.58 & 4.15 & 2.07 & 1.41 \\
\hline & 1986 & 7.90 & 70.09 & 14.25 & 4.09 & 2.15 & 1.51 \\
\hline & 1987 & 8.12 & 71.44 & 13.87 & 3.44 & 1.77 & 1.36 \\
\hline & 1988 & 7.65 & 70.18 & 14.78 & 3.95 & 1.98 & 1.44 \\
\hline & 1989 & 7.96 & 73.08 & 13.59 & 2.73 & 1.39 & 1.24 \\
\hline & 1990 & 7.82 & 72.46 & 14.23 & 2.78 & 1.42 & 1.29 \\
\hline & 1991 & 7.69 & 71.69 & 14.97 & 2.88 & 1.41 & 1.35 \\
\hline & 1992 & 7.47 & 70.65 & 15.77 & 3.15 & 1.52 & 1.44 \\
\hline & 1993 & 6.99 & 70.01 & 16.63 & 3.24 & 1.55 & 1.59 \\
\hline & 1994 & 5.10 & 68.70 & 19.20 & 3.61 & 1.63 & 1.78 \\
\hline & 1995 & 6.89 & 67.60 & 18.32 & 3.72 & 1.69 & 1.77 \\
\hline & 1996 & 6.63 & 66.43 & 19.14 & 4.10 & 1.79 & 1.91 \\
\hline & 1997 & 6.23 & 65.66 & 19.98 & 4.31 & 1.84 & 1.96 \\
\hline & 1998 & 5.85 & 64.83 & 20.67 & 4.58 & 1.97 & 2.10 \\
\hline & 1999 & 5.43 & 62.88 & 21.80 & 5.37 & 2.28 & 2.24 \\
\hline & 2000 & 5.27 & 62.27 & 22.24 & 5.56 & 2.33 & 2.31 \\
\hline & 2001 & 4.17 & 60.36 & 23.97 & 6.25 & 2.65 & 2.60 \\
\hline & 2002 & 3.81 & 59.34 & 24.54 & 6.73 & 2.81 & 2.78 \\
\hline \multirow[t]{19}{*}{ Mulheres } & 1984 & 33.36 & 51.70 & 10.07 & 3.20 & 1.23 & 0.44 \\
\hline & 1985 & 33.76 & 52.29 & 9.45 & 2.94 & 1.15 & 0.42 \\
\hline & 1986 & 33.92 & 52.26 & 9.19 & 3.00 & 1.18 & 0.45 \\
\hline & 1987 & 34.27 & 53.20 & 8.62 & 2.53 & 0.97 & 0.41 \\
\hline & 1988 & 32.72 & 53.34 & 9.54 & 2.86 & 1.09 & 0.43 \\
\hline & 1989 & 34.71 & 54.34 & 7.89 & 1.94 & 0.75 & 0.36 \\
\hline & 1990 & 33.94 & 54.66 & 8.29 & 1.96 & 0.78 & 0.37 \\
\hline & 1991 & 33.39 & 54.51 & 8.90 & 2.03 & 0.77 & 0.41 \\
\hline & 1992 & 32.33 & 54.74 & 9.48 & 2.23 & 0.81 & 0.41 \\
\hline & 1993 & 31.38 & 55.03 & 9.93 & 2.30 & 0.89 & 0.47 \\
\hline & 1994 & 31.20 & 54.29 & 10.58 & 2.47 & 0.96 & 0.50 \\
\hline & 1995 & 30.86 & 53.80 & 11.05 & 2.65 & 1.09 & 0.55 \\
\hline & 1996 & 30.16 & 53.47 & 11.67 & 2.94 & 1.16 & 0.60 \\
\hline & 1997 & 28.96 & 53.89 & 12.19 & 3.06 & 1.17 & 0.74 \\
\hline & 1998 & 28.01 & 53.86 & 12.78 & 3.32 & 1.29 & 0.74 \\
\hline & 1999 & 26.13 & 53.73 & 14.03 & 3.89 & 1.51 & 0.72 \\
\hline & 2000 & 25.02 & 54.10 & 14.57 & 4.03 & 1.54 & 0.74 \\
\hline & 2001 & 21.27 & 55.38 & 16.10 & 4.64 & 1.79 & 0.83 \\
\hline & 2002 & 19.64 & 55.70 & 16.86 & 4.99 & 1.93 & 0.88 \\
\hline
\end{tabular}

Tabela B.3: Porcentagen de casamentos por faixa etária e sexo. Fonte: IBGE 


\section{Referências Bibliográficas}

Aitchinson(1986) J. Aitchinson. The Statistical Analysis of Compositional Data. Chapman and Hall. Citado na pág. 2, 13, 15, 51, 55

Aitchison(1982) J. Aitchison. The statistical analysis of ompositiona data. Journal of the Royal Statistical Society, 44:139-177. Citado na pág. 13, 14

Aitchison e Shen(1980) J. Aitchison e S. M. Shen. Logistic-normal distributions: Some properties and uses. Biometrika, 67:261-272. Citado na pág. 13

Aitchison et al.(2000) J. Aitchison, C. Barceló-Vidal, J. A. Martín-Fernández, e V. PawlowskyGlahn. Logratio analysis and compositional distance. Mathemathical Geology, 32:271-275. Citado na pág. 15,24

Campbell e Mosimann(1987) G. Campbell e J. Mosimann. Multivariate methods for proportional shape. Em ASA Proceedings of the Section on Statistical Graphics, páginas 10-17. Citado na pág. 2,6

Coakley e Rust(1968) J. P. Coakley e B. R. Rust. Sedimentation in a arctic lake. Sedimentary Petrology, 38:1290-1300. Citado na pág. 2, 55

DeGroot e Schervish(2002) M. H. DeGroot e M. J. Schervish. Probability and Statistics. Addison - Wesley, terceira edição. Citado na pág. 45

Draper e Smith(1998) N. R. Draper e H. Smith. Applied regression analysis. John Willwy \& Sons, Inc., terceira edição. Citado na pág. 39

Evans(1997) M. Evans. Bayesian inference procedures via the concept of relative surprise. Communications in Statistics, 26:11251143. Citado na pág. 45

Hijazi e Jernigan(2009) H. H. Hijazi e R. W. Jernigan. Modeling compositional data using dirichlet regression models. Journal of Applied Probability 83 Statistics, 4:77-91. Citado na pág. 7, 25,51

Krätschmer(2007) V. Krätschmer. The uniqueness of extremum estimation. Statistics 6 P Probability Letters, 77:942-951. Citado na pág. 11

Madruga et al.(2003) M.R. Madruga, C. A. B. Pereira, e J. M. Stern. Bayesian evidence test for precise hypotheses. Journal of Statistical Planning and Inference, 117:185-198. Citado na pág. 45

Martin et al.(2011) A. D. Martin, K. M. Quinn, e J. H. Park. Mcmcpack: Markov chain monte carlo in r. Journal of Statistical Software: http://www.jstatsoft.org/v42/i09/, 42:1-21. Citado na pág. 47

Melo et al.(2009) T. F.N. Melo, K. L.P. Vasconcellos, e A. J. Lemonte. Some restriction tests in a new class of regression models for proportions. Computational Statistics and Data Analysis, 53: 3972-3979. Citado na pág. 2, 10, 39, 51 
Pawitan(2001) Y. Pawitan. In all Likelihood: Statistical Modelling and Inference Using Likelihood. Oxford University Press. Citado na pág. 43, 46

Pereira e Stern(2008) C. A. B. Pereira e J. M. Stern. Special characterizations of standart discrete models. Revstat, 6:199-230. Citado na pág. 2, 5, 7, 8

Pereira e Stern(1999) C. A. B. Pereira e J. M. Stern. Evidence and credibilidty: Full bayesian significance test of precise hypothesis. Entropy Journal, 1:99-110. Citado na pág. 45

Pereira et al.(2008) C. A. B. Pereira, J. M. Stern, e S. Wechsler. Can a significant test be genuinely bayesian. Bayesian Analysis, 3:79-100. Citado na pág. 45

Robert e Casella(2004) C. P. Robert e G. Casella. Monte Carlo Statistical Methods. Springer, segunda edição. Citado na pág. 46

Ronning(1989) G. Ronning. Maximum likelihood estimation of dirichlet distributions. Journal of Statistical Computation and Simulation, 32:215-221. Citado na pág. 5

Schervish(1997) M. J. Schervish. Theory of Statistics. Springer. Citado na pág. 45

Schwarz(1978) G. Schwarz. Estimating the dimension of a model. Annals of Statistics, 6:461-464. Citado na pág. 39, 43

Stern(2008) J. M. Stern. Tutorial: Cognitive Construtivism and the Epistemic Significance of Sharp Statistical Hypotheses. 28th International Workshop on Bayesian Inference and Maximum Entropy Methods in Science and Engeneering, Boracéia-Brazil. Citado na pág. 41

Wang et al.(2007) H. Wang, Q. Liu, H. M.K. Mok, L. Fu, e W. M. Tse. A hyperspherical transformation forecasting model for compositional data. European Journal of Operational Research, 179:459-468. Citado na pág. 2, 18, 51

Watkins(2002) D. S. Watkins. Fundamentals of Matrix Computations. John Wiley \& Sons. Citado na pág. 54

Wilks(1938) S. S. Wilks. The large-sample distribution of the likelihood ratio for testing composite hypotheses. Annals of Mathematical Statistics, 9:60-62. Citado na pág. 46 


\section{Índice Remissivo}

dado composicional, 1

distribuição

de Dirichlet, 5, 6

normal, 13

Familia Exponencial, 5

função

de verossimilhança, 7

isomorfismo linear, 15

matriz

de modelo, 6

norma

euclidiana, 8

processos de Bernoulli, 9

regressão, 1

regressão

de Dirichlet, 5

linear, 6

Simplex, 1

transformação

Log-esférica, 22

Logaritmo da razão, 13

Tangente-esférica, 22

transformações

equivalentes, 15

logísticas, 15

verossimilhança, 8 